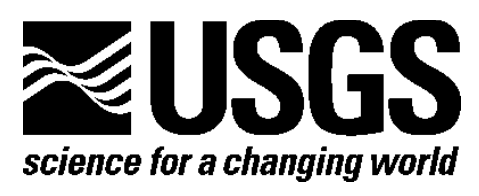

\title{
Thermodynamic Method for Generating Random Stress Distributions on an Earthquake Fault
}

By Michael Barall, Invisible Software, Inc., and Ruth A. Harris, U.S. Geological Survey

Open-File Report 2012-1226

U.S. Department of the Interior

U.S. Geological Survey 


\section{U.S. Department of the Interior \\ KEN SALAZAR, Secretary}

\section{U.S. Geological Survey \\ Marcia K. McNutt, Director}

U.S. Geological Survey, Reston, Virginia: 2012

For more information on the USGS-the Federal source for science about the Earth, its natural and living resources, natural hazards, and the environment-visit http://www.usgs.gov or call 1-888-ASK-USGS

For an overview of USGS information products, including maps, imagery, and publications, visit $h$ ttp://www.usgs.gov/pubprod

To order this and other USGS information products, visit http://store.usgs.gov

Suggested citation:

Barall, Michael, and Harris, R.A., 2012, Thermodynamic method for generating random stress distributions on an earthquake fault: U.S. Geological Survey Open-File Report 2012-1226, 112 p.

Any use of trade, product, or firm names is for descriptive purposes only and does not imply endorsement by the U.S. Government.

Although this report is in the public domain, permission must be secured from the individual copyright owners to reproduce any copyrighted material contained within this report. 


\section{Contents}

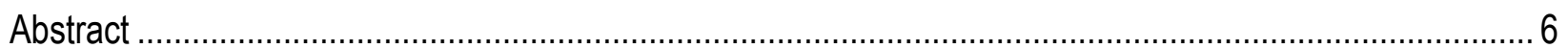

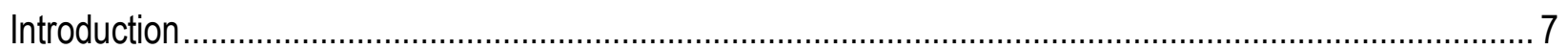

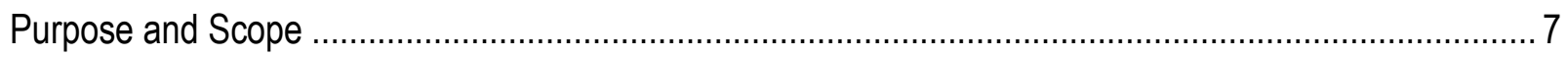

Previous Work

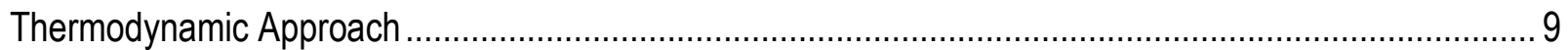

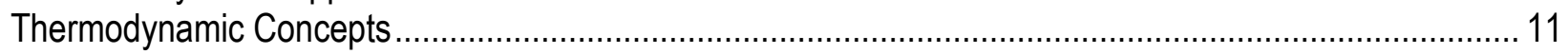

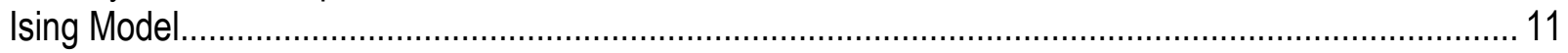

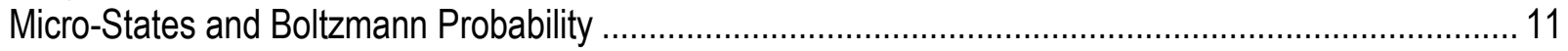

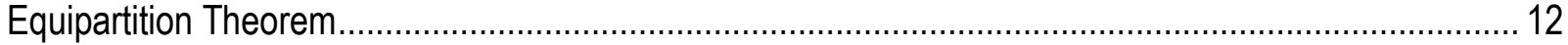

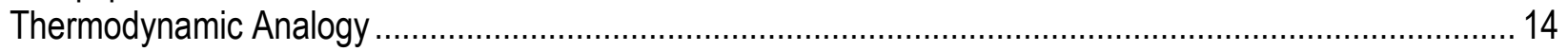

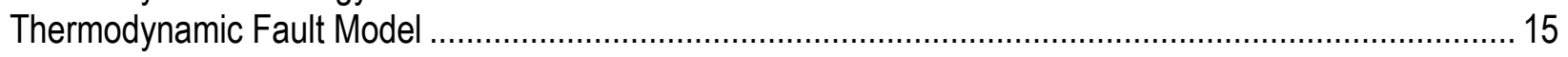

Basis Displacement Fields ................................................................................................. 15

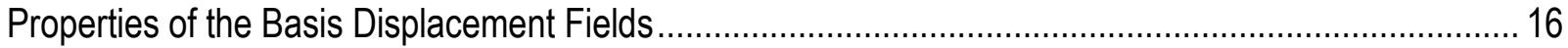

Thermodynamic Model Construction......................................................................................... 18

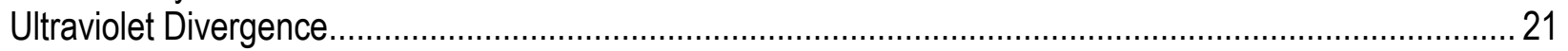

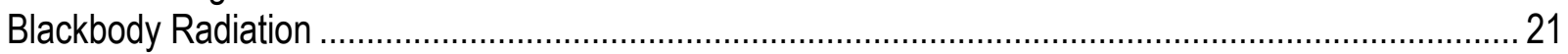

Finite Fault Strength Restricts the Set of Accessible States ………………………………......... 22

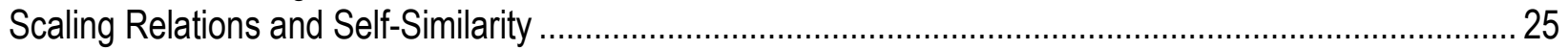

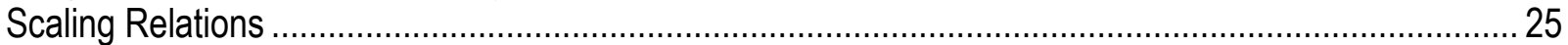

Energy Rolloff and Power Spectrum ........................................................................................ 27

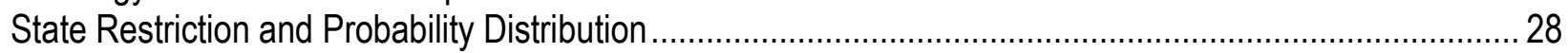

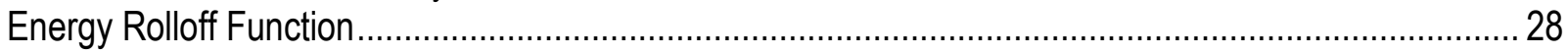

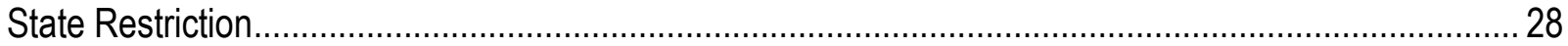

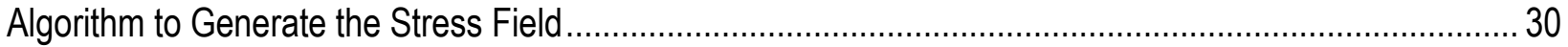

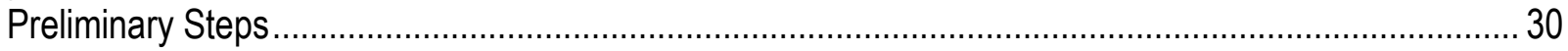

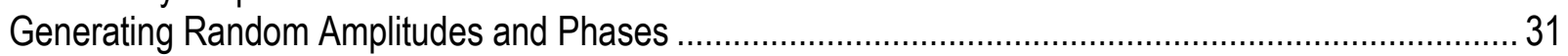

Summing the Random Stress Fields..................................................................................... 32

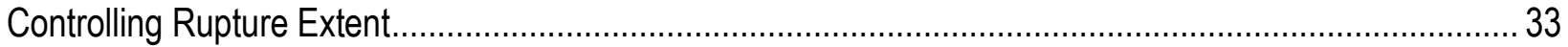

Depth Conditioning ............................................................................................................... 33

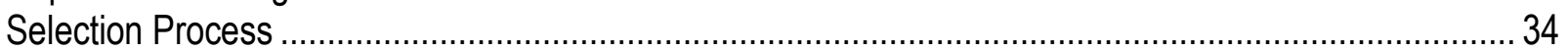

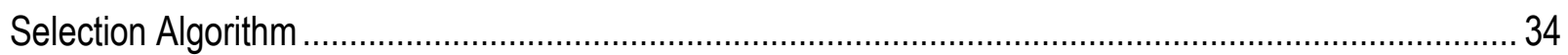

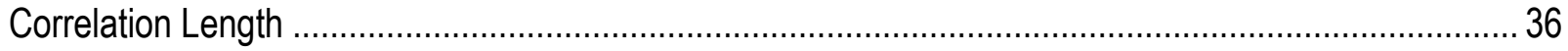

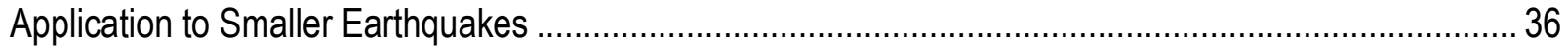

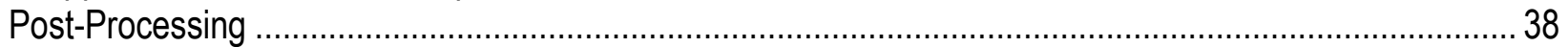

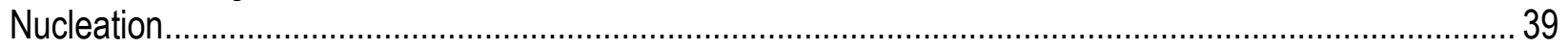

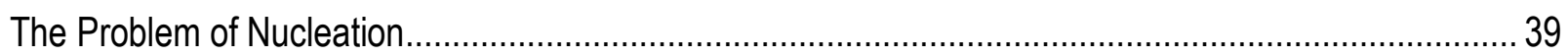

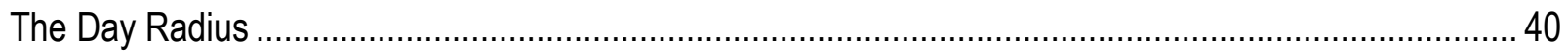

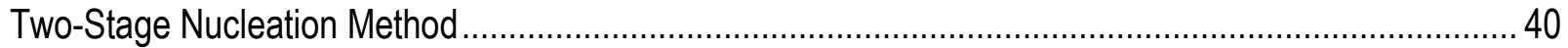

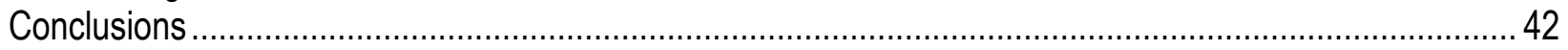

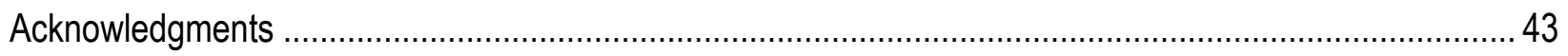




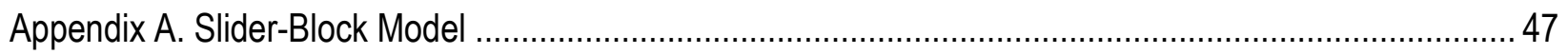

Appendix B. Properties of the Basis Displacement Fields ...............................................................49

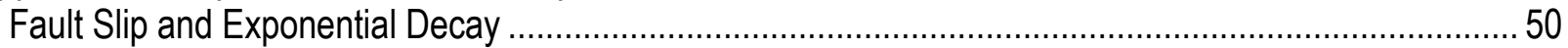

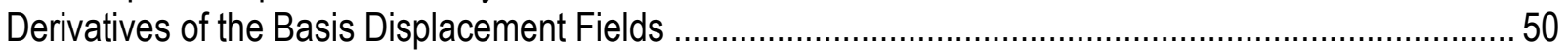

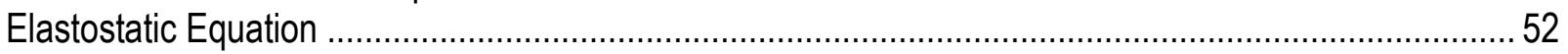

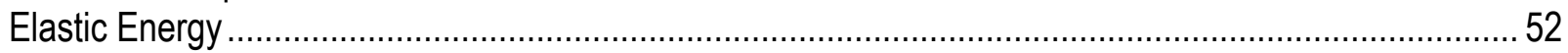

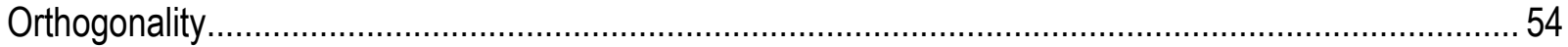

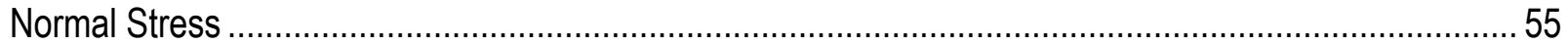

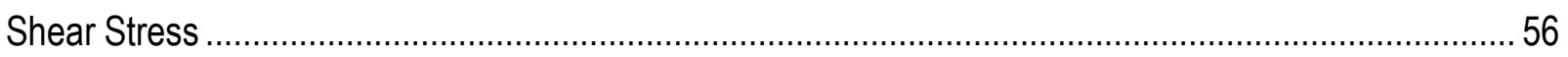

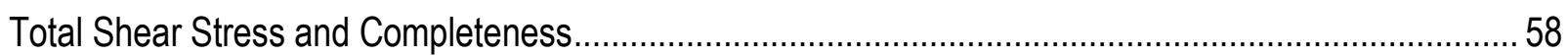

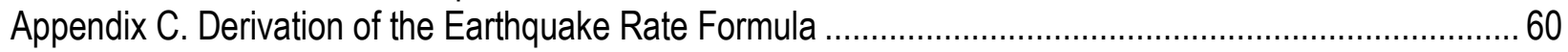

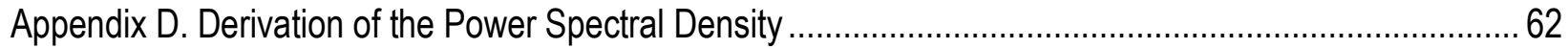

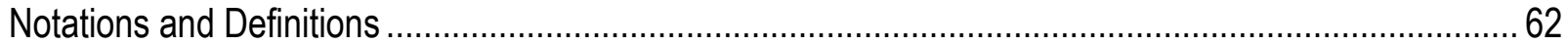

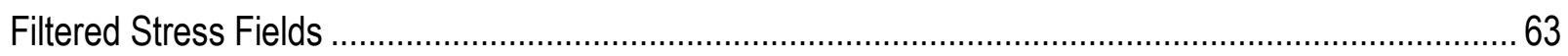

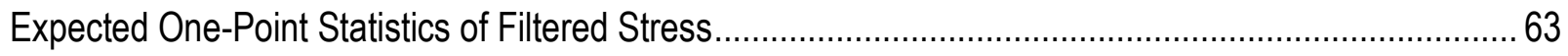

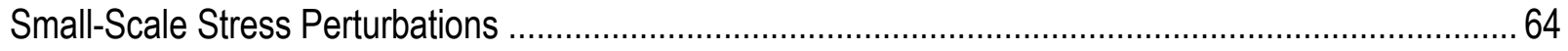

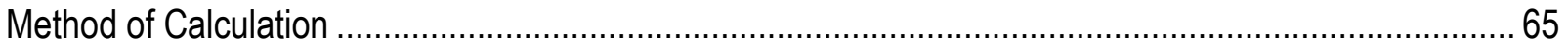

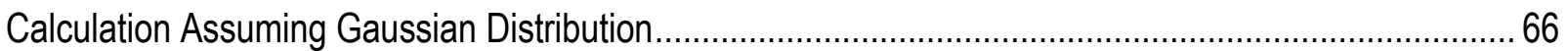

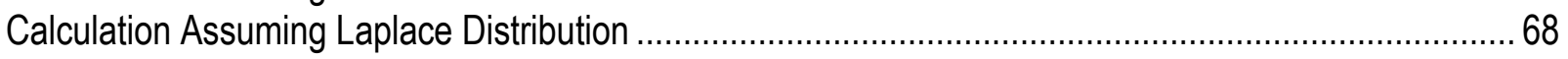

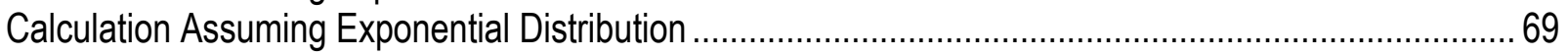

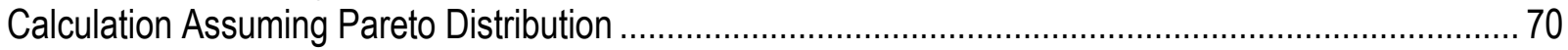

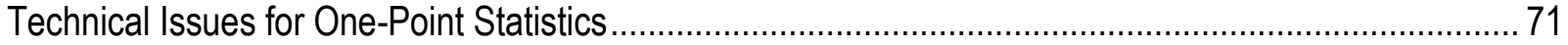

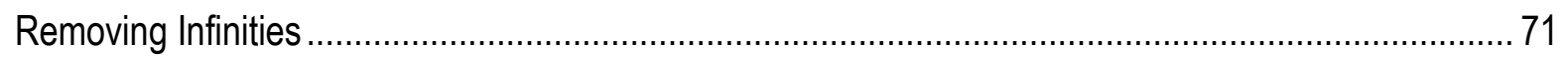

Stress Distributions and Wave Amplitudes.............................................................................. 72

Fault Surface and Mean Values ............................................................................................ 73

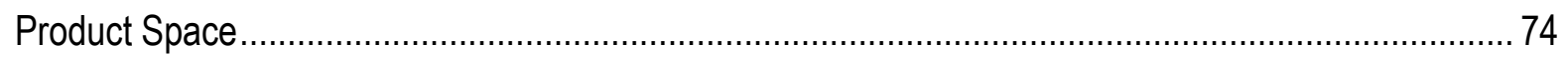

Definition of Expected One-Point Statistics .......................................................................... 74

Mean and Variance of Expected One-Point Statistics .................................................................... 76

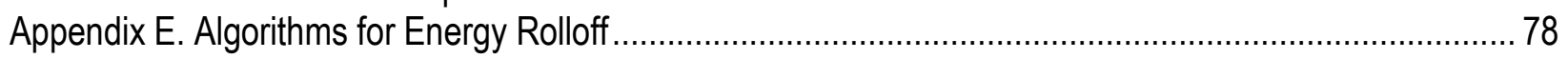

Energy Rolloff Function ........................................................................................................ 78

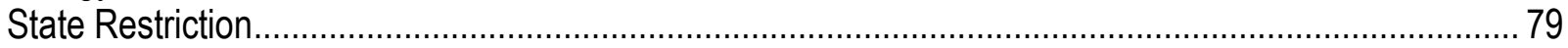

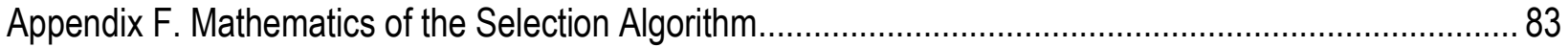

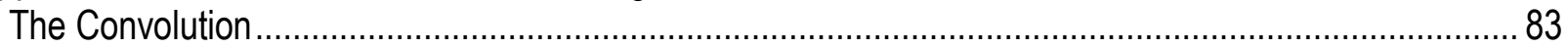

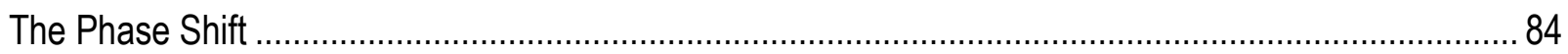

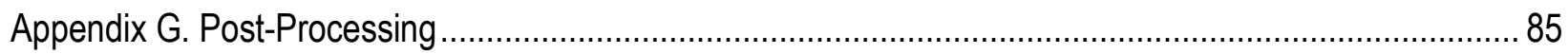

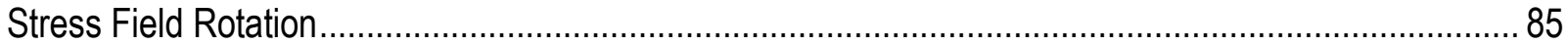

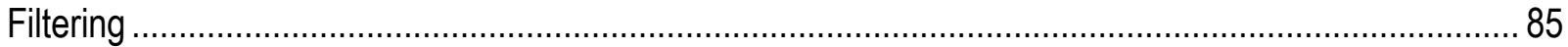

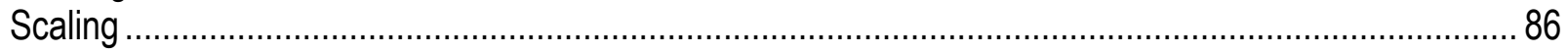

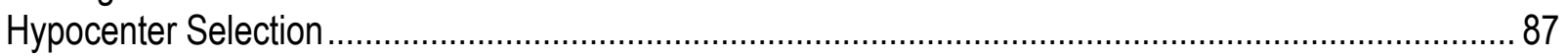

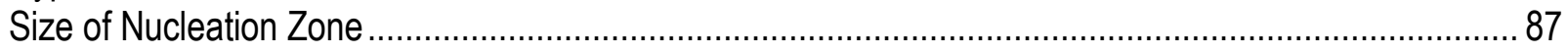

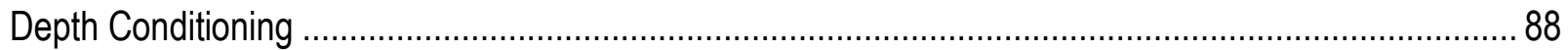

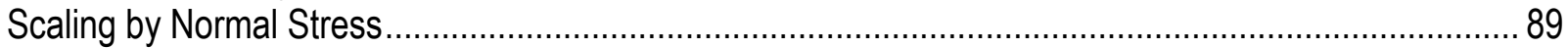

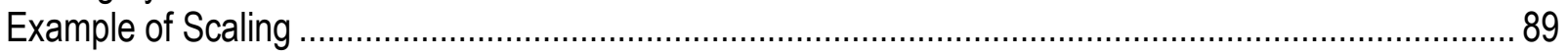

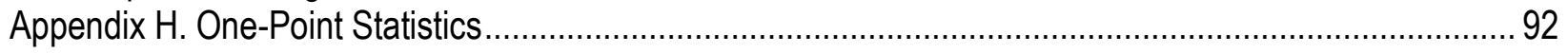

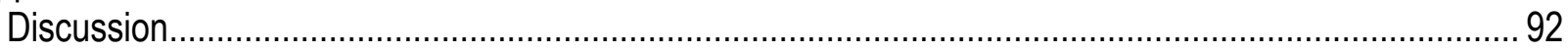




\section{Figures}

Figure 1. Components of dynamic and kinematic earthquake simulations. ..........................................100

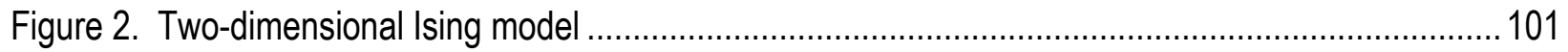

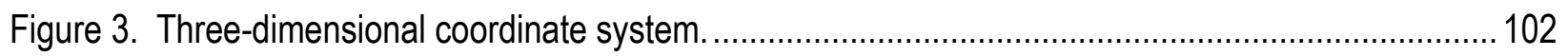

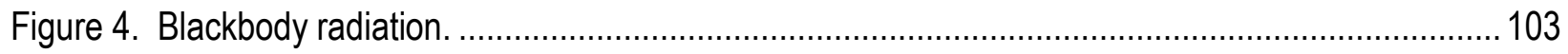

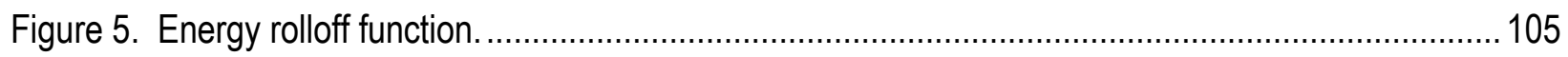

Figure 6. Examples of random stress distributions generated by our method. ......................................107

Figure 7. Selection process for controlling rupture extent along-strike. .............................................. 108

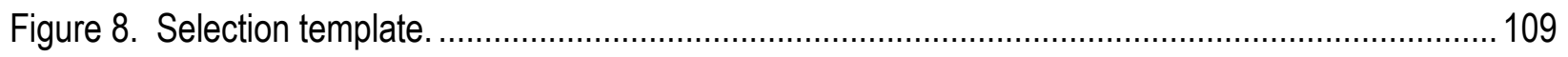

Figure 9. Effect of varying the correlation length.............................................................................111

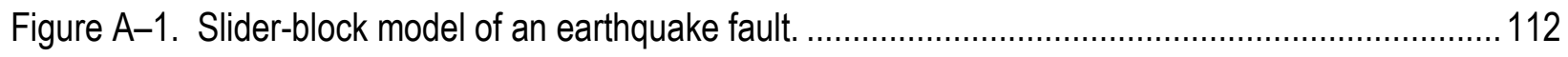

\section{Tables}

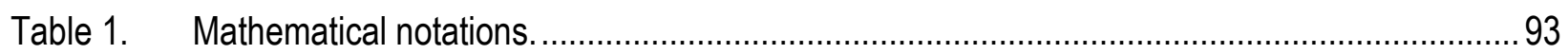

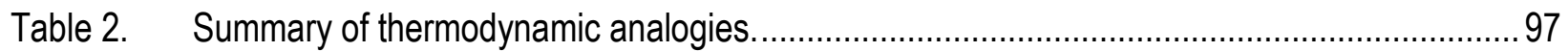

\section{Conversion Factors}

SI to Inch/Pound

\begin{tabular}{lll}
\hline \multicolumn{1}{c}{ Multiply } & By & To obtain \\
\hline meter $(\mathrm{m})$ & Length & \\
kilometer $(\mathrm{km})$ & 3.281 & foot $(\mathrm{ft})$ \\
& 0.6214 & mile $(\mathrm{mi})$ \\
\hline megapascal $(\mathrm{MPa})$ & Pressure & \\
\hline
\end{tabular}




\title{
Thermodynamic Method for Generating Random Stress Distributions on an Earthquake Fault
}

Michael Barall, Invisible Software, Inc., and Ruth A. Harris, U.S. Geological Survey

\begin{abstract}
This report presents a new method for generating random stress distributions on an earthquake fault, suitable for use as initial conditions in a dynamic rupture simulation. The method employs concepts from thermodynamics and statistical mechanics. A pattern of fault slip is considered to be analogous to a micro-state of a thermodynamic system. The energy of the micro-state is taken to be the elastic energy stored in the surrounding medium. Then, the Boltzmann distribution gives the probability of a given pattern of fault slip and stress. We show how to decompose the system into independent degrees of freedom, which makes it computationally feasible to select a random state. However, due to the equipartition theorem, straightforward application of the Boltzmann distribution leads to a divergence which predicts infinite stress. To avoid equipartition, we show that the finite strength of the fault acts to restrict the possible states of the system. By analyzing a set of earthquake scaling relations, we derive a new formula for the expected power spectral density of the stress distribution, which allows us to construct a computer algorithm free of infinities. We then present a new technique for controlling the extent of the rupture by generating a random stress distribution thousands of times larger than the fault surface, and selecting a portion which, by chance, has a positive stress perturbation of the desired size. Finally, we present a new two-stage nucleation method that combines a small zone of forced rupture with a larger zone of reduced fracture energy.
\end{abstract}




\section{Introduction}

Dynamic earthquake simulations are powerful computational tools. They incorporate our knowledge of earthquake physics and allow for the prediction of earthquake magnitudes and effects. These simulations also require many assumptions about how faults and earthquakes work, including values for the initial stress conditions, and parameters that describe the fault geometry, the frictional failure criterion, and the material properties (for example, Harris (2004)).

Fault geometry and material properties can, in principle, be estimated given adequate geophysical measurements, but other aspects of dynamic earthquake rupture remain more elusive. The most challenging parameters are those that relate to fault friction and the initial stress conditions on faults. We leave fault friction for others to study and refer the reader to overviews by Tullis (2007), Lapusta (2009), and Bizzarri (2011).

In this report we concentrate on the problem of defining appropriate probability distributions for fault stress heterogeneity. We present a method for prescribing random realizations of stress at the onset of dynamic earthquake rupture simulations. Preliminary tests of this approach show the resulting heterogeneous initial-stress distributions to operate well in computer codes that are included in the Southern California Earthquake Center (SCEC) Dynamic Rupture Code group (http://scecdata.usc.edu/cvws; Harris, Barall, Archuleta, and others (2009); Harris, Barall, Andrews, and others (2011)). Computer code to implement the method accompanies this report.

\section{Purpose and Scope}

We are concerned with setting up the initial conditions for a dynamic rupture simulation. Detailed examples of initial conditions for dynamic rupture simulations are available on the SCEC website (http://scecdata.usc.edu/cvws). To perform a dynamic rupture simulation, one must specify:

- Fault geometry.

- Rock properties (which we presume to be linear elastic).

- Frictional constitutive properties of the fault (which may have parameters that vary with position on the fault surface).

- Initial normal stress at each point on the fault surface.

- Initial shear stress at each point on the fault surface.

In this work, we give a method for randomly generating the initial shear stresses. All the other components listed above are presumed to be specified in advance.

Our method addresses the case of a planar fault, embedded in a uniform linear elastic half-space, with uniform orientations of the initial shear stress. For ease of discussion, we describe the method for the case of a vertical planar fault with horizontal initial shear stresses. There is nothing in the development of the method that relies on the fault being vertical or the shear stress orientation being horizontal, and so the method can be applied unchanged to dipping faults and to faults with non-horizontal (uniform) initial shear stress orientations.

For ease of discussion, we also assume that the simulated earthquake is large enough to rupture the full depth of the seismogenic zone and that the fault obeys a linear slip-weakening friction law. For the case of earthquakes too small to rupture the full depth of the seismogenic 
zone, a minor modification to the method is required, which we describe. The method does not depend on details of the friction law and so may be applied unchanged to other friction laws.

We do not address the cases of non-planar faults, non-uniform initial shear stress orientations, or non-uniform linear elastic rock properties. One can of course apply our method (or any other method) to such cases simply by generating an initial shear stress distribution for a similar case where the method is applicable, and then just using that stress distribution despite the fact that the method employed is not directly applicable to the case at hand. In doing so, however, one must be aware that the method is being employed in a situation where it is not specifically applicable.

Our method cannot be used for plastic rock properties, because that would require a randomly generated initial stress tensor throughout the three-dimensional medium. Our method only generates a random initial shear stress distribution on the fault surface.

\section{Previous Work}

Heterogeneous fault-stress distributions and heterogeneous fault-slip distributions have been studied for at least 35 years, including early work by Yamashita (1976), Das and Aki (1977), Mikumo and Miyatake (1978), Hanks (1979), McGarr and others (1979), Andrews (1980; 1981), Boatwright (1981), and Day (1982). More recent studies include those by Beroza and Mikumo (1996), Bouchon (1997), Day and others (1998), Mai and Beroza (2002), Oglesby and Day (2002), Guatteri and others (2003), Guatteri, Mai, and Beroza (2004), Lavallée and others (2006), Ripperger and others (2007), Ripperger, Mai, and Ampuero (2008), Aagaard and Heaton (2008), Schmedes et al. (2010), and Andrews and Barall (2011), among others. Some of these studies have parameterized the fault slip (or slip velocity), others the fault stress. The former is needed for kinematic models of earthquake rupture, in which the timing and amount of slip at each point on a fault are pre-determined before the start of the simulation. The latter is required for spontaneous models of dynamic earthquake rupture, in which the rupture extent and the timing and amount of slip are determined by the physics of the processes occurring during the course of the simulation (fig. 1). Among the authors who have worked with heterogeneous initial stresses in spontaneous rupture simulations, the need for heterogeneity has been explained as necessary to produce reasonable rupture propagation direction and fault slip patterns (for example, Yamashita, 1976), and as an explanation for variable rupture speeds and spatial variations of peak slip velocity (for example, Day, 1982). Some authors have proposed that the heterogeneity may reside in the initial, static, or dynamic frictional stresses or all three ( for example, Mikumo and Miyatake, 1978; Peyrat and others, 2001; Bouchon, 1997; Mavroeidis and Papageorgiou, 2010), with Mikumo and Miyatake (1978) proposing that the heterogeneity may be due to geometrical fault complexity and may explain observations of high-frequency radiation patterns and locations of aftershocks. Many have mentioned that heterogeneity in stresses is essential for multi-earthquake cycle simulations.

This report does not assume that we know the exact cause of spatial fluctuations in the initial stresses on faults, it just recognizes that realistic ground motions are unlikely to be produced by assuming constant slip or constant initial stress conditions on a planar fault surface. We therefore provide a formulation to describe the heterogeneity. As we improve our understanding of the fine-scale details of faults, along with our ability to realistically simulate them ( for example, Kaneko and others, 2010; Shi and Day, 2011), the approach presented here may evolve. For now, however, we need to adopt a stochastic method to generate heterogeneities in the initial stress state in dynamic, spontaneous earthquake rupture simulations. 


\section{Thermodynamic Approach}

Many existing methods for generating random stress distributions attempt to emulate statistical properties of earthquakes inferred from slip inversion models (for example, Mai and Beroza, 2002; Lavallée and others 2006), or from considerations of earthquake self-similarity (for example, Andrews and Barall, 2011). Here we explore a new approach, which is to generate random stress distributions using concepts and tools drawn from thermodynamics and statistical mechanics. Our approach is motivated by the two-dimensional Ising model, which is an example of how a physical system with simple laws can self-organize into large structures. Smalley and others (1985) and Huang and others (1998) construct models of stress transfer on an earthquake fault with features analogous to the Ising model. Other authors who have applied statistical mechanics concepts to the study of earthquake faults include Rundle and others (2003) and Abaimov (2009). Here we employ an analogy to the Ising model to construct a method for generating random heterogeneous initial stress.

In statistical mechanics, it is presumed that the precise state of a physical system is unknowable, so one must talk about a probability distribution of states. The probability that a system is in a given state depends on the state's energy; the higher the energy, the lower the probability. We consider a pattern of slip on a fault surface to be analogous to a thermodynamic state. Fault slip distorts the three-dimensional elastic medium surrounding the fault, and we consider the resulting elastic energy to be analogous to the energy of a thermodynamic state. The goal of this report is to carry this analogy through to obtain a probability distribution for the resulting patterns of stress on the fault.

Our work begins with a review of the two-dimensional Ising model and the thermodynamic concepts we need. Then, we introduce a set of three-dimensional displacement fields, which provide a mathematically tractable way to describe patterns of fault slip and stress, and the resulting distortion of the three-dimensional elastic medium. The displacement fields have the property of orthogonality, which means that when combined in a linear superposition their elastic energies are additive. So, these displacement fields are analogous to the degrees of freedom of a thermodynamic system, and we can use the equipartition theorem to develop a probability distribution.

Unfortunately, the equipartition theorem predicts infinitely large stresses due to the everincreasing contributions of short-wavelength displacement fields. This phenomenon, called ultraviolet divergence, occurs in some statistical mechanics problems such as the calculation of the spectrum of blackbody radiation. Its resolution is to restrict the possible states of the system. For us, the finite strength of the fault restricts the possible patterns of fault slip and stress, because a stress pattern that exceeds the yield strength of the fault cannot occur. By careful consideration of earthquake scaling relations and self-similarity, we derive a formula showing how the finite fault strength causes the energy distribution to deviate from equipartition. This allows us to develop a probability distribution that predicts finite levels of stress. We then present an algorithm for generating random realizations of initial stress according to this probability distribution.

After generating a random stress field, several post-processing steps are required to make it suitable for use in a dynamic rupture simulation. It is desirable to make the simulated rupture stop spontaneously by encountering low stress outside the intended rupture area. We achieve this using a new method, which generates a random stress field over an extremely large area, and then selects a small portion of the random stress field to use on the simulated fault surface, which, just by chance, has the required low-stress regions. Then, we indicate how to scale and 
filter the stress field, to make it consistent with the assumed fault friction law, and to suppress stress fluctuations too small to be resolved by the dynamic rupture code. Finally, we describe a new technique for initiating, or nucleating, the simulated rupture, which combines a small region of forced rupture with a larger region of reduced fracture energy. 


\section{Thermodynamic Concepts}

Our method is based on concepts from thermodynamics, and in particular the twodimensional Ising model. In this section we review the relevant concepts. For a general reference see Reif (1965).

There are several reasons why we have chosen to use thermodynamics as a basis for our method. First, the Ising model is a classic example of how a stochastic physical system can selforganize into large structures. For the Ising model, the large structures are magnetic domains in a ferromagnetic material. For an earthquake fault, the large structures are stress perturbations large enough to sustain a big earthquake. Second, the principles of thermodynamics are broadly applicable to many physical systems, and so we may expect this approach to yield physically reasonable results. And third, this approach lets us utilize known results and concepts from thermodynamic, such as the Boltzmann probability distribution and the equipartition theorem, in the construction of our method.

\section{Ising Model}

The two-dimensional Ising model (Ising, 1925; Onsager, 1944) is a simple model of a magnetic material, such as iron (fig. 2). Each point on a two-dimensional lattice holds a spin that can point in one of two directions, either up or down. Each spin represents, for example, a single atom of iron which behaves like a tiny magnet. The north pole of the magnet can point either up or down. (Quantum mechanics limits the orientation of the spin to one of two discrete directions.) The value of the $n$th spin is denoted by $s_{n}$, which is +1 if the spin points up or -1 if the spin points down.

In the Ising model, each spin interacts only with its four nearest neighbors. (If the lattice is of finite size, then spins on the edge of the lattice have only three or two nearest neighbors. Alternatively, one can impose periodic boundary conditions, in which case every spin on a finite lattice can have four nearest neighbors.) The total energy, $E$, of the system is then given by the formula

$$
E=-\sum_{m n} K s_{m} s_{n}
$$

where the sum runs over nearest-neighbor pairs. For a ferromagnetic material, the coupling constant, $K$, is positive, which makes it energetically favorable for adjacent spins to line up. (For a paramagnetic material, $K$ is negative.) It can be shown that if the temperature is not too high, then the spins in the Ising model spontaneously organize themselves into large domains in which most of the spins are aligned, which in turn can create a macroscopic magnetic field.

\section{Micro-States and Boltzmann Probability}

A micro-state of a system is a complete specification of the system's microscopic state. For example, a micro-state of the Ising model would specify the values of all the spins $\left\{s_{n}\right\}$. In general, one cannot know the micro-state of a system. Instead, one knows macroscopic parameters such as the temperature. Given the values of macroscopic parameters, one then infers a probability distribution for the possible micro-states. 
The Boltzmann probability distribution gives the probability $P(s)$ that the system is in micro-state $s$ :

$$
P(s)=\frac{1}{Z(\beta)} e^{-\beta E(s)} \quad \text { where } \quad \beta \propto \frac{1}{T}
$$

Here $E(s)$ is the energy of the system when it is in micro-state $s$, and $\beta$ is proportional to the reciprocal of the temperature, $T$. The normalization factor $Z(\beta)$ is called the partition function and is defined to be

$$
Z(\beta) \equiv \sum_{s} e^{-\beta E(s)}
$$

where the sum runs over all accessible micro-states $s$. If the micro-states form a continuum, then the sum is replaced with an integral. A useful feature of the partition function is that the expected energy of the system, denoted $\langle E\rangle$, can be computed by differentiating the partition function:

$$
\langle E\rangle \equiv \frac{1}{Z(\beta)} \sum_{s} E(s) e^{-\beta E(s)}=-\frac{1}{Z(\beta)} \frac{d Z}{d \beta}
$$

The Boltzmann distribution can be interpreted as expressing two principles: (1) physical systems tend to assume lower-energy states; and (2) if you pump a certain amount of energy into a physical system (for example, by heating it), then all accessible micro-states with that amount of energy, or less, are about equally likely.

\section{Equipartition Theorem}

The equipartition theorem is a consequence of the Boltzmann distribution (Boltzmann, 1876). Suppose that (1) the system can be decomposed into a set of degrees of freedom, and (2) the total energy of the system is equal to the sum of the energies associated with each degree of freedom. Then, the Boltzmann probability distribution applies separately to each degree of freedom, as if they were independent random variables. So, if $s=\left\{s_{n}\right\}$ is a decomposition of the micro-state into a set of degrees of freedom, and if the energy of the micro-state can be expressed in the form

$$
E(s)=\sum_{n} E_{n}\left(s_{n}\right)
$$

then the probability of finding the system in micro-state $s$ is

$$
P(s)=\prod_{n} P\left(s_{n}\right) \quad \text { where } \quad P\left(s_{n}\right)=\frac{1}{Z_{n}(\beta)} e^{-\beta E_{n}\left(s_{n}\right)}
$$

This equation is the formula for the joint probability distribution of independent random variables. 
Continuing the equipartition theorem, in addition suppose that (3) each degree of freedom is a continuous variable that can assume any real value, and (4) the energy associated with an individual degree of freedom depends quadratically on its value. Then, each degree of freedom has the same expected energy,

$$
\left\langle E_{n}\right\rangle=\frac{1}{2 \beta} \quad \text { for all } n
$$

where $E_{n}=E_{n}\left(s_{n}\right)$ is the energy associated with the $n$th degree of freedom.

Other thermodynamic concepts, including density of states and ultraviolet divergence, will be introduced as needed. 


\section{Thermodynamic Analogy}

Here we give an overview of how we intend to construct a heterogeneous stress pattern by analogy to the Ising model. In the next section we begin to develop the machinery to carry out this plan. The steps to construct the thermodynamic analogy are:

1. The system is the earthquake fault and surrounding rock.

2. A micro-state is defined to be a pattern of slip on the fault.

3. The energy, $E$, of a given micro-state is defined to be the elastic energy associated with the given pattern of slip.

4. Choose a thermodynamic parameter, $\beta$ (This will turn out to be equivalent to choosing a correlation length for the pattern).

5. Use a computer to randomly construct a micro-state, so that the probability of choosing a given pattern of slip equals its Boltzmann probability (which depends on its energy).

6. To make the computation feasible, decompose the system into a set of degrees of freedom, so that the amplitude for each degree of freedom can be selected independently according to the Boltzmann distribution.

7. Compute the pattern of shear stress resulting from the selected pattern of slip.

Table 2 summarizes the analogy between a thermodynamic system and an earthquake fault.

In attempting to carry out this plan, we encounter a major obstacle: the Boltzmann distribution predicts infinitely large stresses. This behavior is known as an ultraviolet divergence. To make the stresses finite, we need to revisit the Gutenberg-Richter relation and the subject of earthquake self-similarity (or scale-invariance), and derive a new result concerning the stress distribution that is implied by self-similarity.

Once the ultraviolet divergence is resolved, the above steps can be used to generate a stress distribution. Then, we show how to control the extent of the rupture, select the correlation length, and nucleate the rupture. 


\section{Thermodynamic Fault Model}

We must work in three dimensions to construct a thermodynamic fault model with correct energy spectrum. (Appendix A explains why a two-dimensional fault model cannot be used.) To work in three dimensions, we introduce a set of three-dimensional displacement fields, which are approximate solutions to the problem of fault slip in an elastic medium. We can then describe a three-dimensional system configuration by forming a linear combination of the displacement fields. These fields are orthogonal, which means that the elastic energy associated with a linear combination of displacement fields equals the sum of the elastic energies of the individual fields. Due to their orthogonality, these fields act as the degrees of freedom of a thermodynamic system.

\section{Basis Displacement Fields}

The setting is a vertical, strike-slip fault in a three-dimensional elastic full-space (fig. 3 ). Points in space are denoted by coordinates $\left(x_{0}, x_{1}, x_{2}\right)$ where the $x_{0}$ coordinate is vertical, the $x_{1}$ coordinate is parallel to the fault strike, and the $x_{2}$ coordinate is perpendicular to the fault. The fault plane is $x_{2}=0$.

We impose periodic boundary conditions in the $x_{0}$ and $x_{1}$ directions (but not in the $x_{2}$ direction). Let $L_{0}$ and $L_{1}$ be the periods in the $x_{0}$ and $x_{1}$ directions, respectively. Then the fault area is $L_{0} L_{1}$. defined as:

A displacement is represented by a vector $\left(u_{0}, u_{1}, u_{2}\right)$. Our basis displacement fields are

$$
\begin{gathered}
u_{0}\left(x_{0}, x_{1}, x_{2}\right)=-F\left(k_{0}, k_{1}\right) \frac{k_{0} k_{1}}{k^{2}+k_{0}^{2}} e^{-k\left|x_{2}\right|} \cos \left(k_{0} x_{0}+k_{1} x_{1}+\Phi\left(k_{0}, k_{1}\right)\right) \operatorname{sgn}\left(\mathrm{x}_{2}\right) \\
u_{1}\left(x_{0}, x_{1}, x_{2}\right)=F\left(k_{0}, k_{1}\right) e^{-k\left|x_{2}\right|} \cos \left(k_{0} x_{0}+k_{1} x_{1}+\Phi\left(k_{0}, k_{1}\right)\right) \operatorname{sgn}\left(x_{2}\right) \\
u_{2}\left(x_{0}, x_{1}, x_{2}\right)=-F\left(k_{0}, k_{1}\right) \frac{k k_{1}}{k^{2}+k_{0}^{2}} e^{-k\left|x_{2}\right|} \sin \left(k_{0} x_{0}+k_{1} x_{1}+\Phi\left(k_{0}, k_{1}\right)\right) .
\end{gathered}
$$

Here $F\left(k_{0}, k_{1}\right)$ is an arbitrary amplitude, $\Phi\left(k_{0}, k_{1}\right)$ is an arbitrary phase, $k_{0}$ and $k_{1}$ are wavenumbers in the $x_{0}$ and $x_{1}$ directions, and $k$ is the scalar wavenumber defined as:

$$
k=\sqrt{k_{0}^{2}+k_{1}^{2}}
$$

To understand the structure of these basis displacement fields, take a closer look at the formula for $u_{1}$, which is displacement in the along-strike direction. It is a product of four terms:

- An arbitrary amplitude $F\left(k_{0}, k_{1}\right)$.

- A term $e^{-k\left|x_{2}\right|}$ which decays exponentially with increasing distance from the fault, over a characteristic distance $1 / k$.

- A term $\cos \left(k_{0} x_{0}+k_{1} x_{1}+\Phi\left(k_{0}, k_{1}\right)\right)$ which is a sinusoidal variation in the fault plane, with wavelength $2 \pi / k$. 
- A term $\operatorname{sgn}\left(x_{2}\right)$ which changes sign when crossing the fault plane $x_{2}=0$, producing a discontinuity at the fault plane which represents fault slip.

The formulas for $u_{0}$ and $u_{2}$ are needed to satisfy the elastostatic equation and to make the shear stress point along-strike. This is discussed further below.

\section{Properties of the Basis Displacement Fields}

We now discuss the properties of the basis displacement fields. Mathematical proofs are in appendix B. Mathematical notations are summarized in table 1.

Property 1. Away from the fault, the displacements are an exact solution of the elastostatic equation:

$$
\begin{gathered}
\sigma_{i j, j}=0 \\
\sigma_{i j}=\lambda \delta_{i j} \varepsilon_{l l}+2 \mu \varepsilon_{i j} \\
\varepsilon_{i j}=\frac{1}{2}\left(u_{j, i}+u_{i, j}\right) .
\end{gathered}
$$

Here $\sigma_{i j}$ is the stress tensor, $\varepsilon_{i j}$ is the strain tensor, $\mu$ is the shear modulus, and $\lambda$ is the volumetric Lame modulus.

Property 2. The shear stress, $\tau$, is the same on both sides of the fault, as it should be.

Property 3. The normal stress, $\sigma$, is not the same on both sides of the fault. This is what makes the displacement field an approximate, rather than exact, solution to the elastostatic fault slip problem.

Property 4. At the fault, the displacements describe fault slip which varies sinusoidally, with wavenumbers $k_{0}$ and $k_{1}$ in the along-dip and along-strike directions, and with amplitude proportional to $F=F\left(k_{0}, k_{1}\right)$.

Property 5. Away from the fault, the displacements die off exponentially over characteristic distance $1 / k$.

This shows that fault slip with long wavelength (small $k$ ) penetrates more deeply into the three-dimensional medium than fault slip with short wavelength (large $k$ ).

Property 6. The elastic energy in the three-dimensional medium is:

$$
E\left(k_{0}, k_{1}\right)=\frac{1}{2} \mu L_{0} L_{1}\left(\frac{2 k^{2}}{k^{2}+k_{0}^{2}}\right)^{2} k F\left(k_{0}, k_{1}\right)^{2}
$$

This is the expected energy spectrum, with $E \propto k F^{2}$.

Property 7. The displacement fields are orthogonal. If you form a linear combination of these displacement fields, with different wave vectors, then the total elastic energy, $E_{\text {total }}$, of the linear combination equals the sum of the energies of the individual fields:

$$
E_{\text {total }}=\frac{L_{0} L_{1}}{4 \pi^{2}} \int E\left(k_{0}, k_{1}\right) d k_{0} d k_{1}
$$


Orthogonality makes the basis displacement fields satisfy the premises of the equipartition theorem. This is discussed below.

Remark: At this point we pause to explain the meaning of an integral over wavenumber space, such as the one shown above. Because we impose periodic boundary conditions, the wavenumbers are restricted to a set of discrete values

$$
k_{0}=\frac{2 \pi n_{0}}{L_{0}} \quad \text { and } \quad k_{1}=\frac{2 \pi n_{1}}{L_{1}}
$$

where $n_{0}$ and $n_{1}$ are integers. An integral over wavenumbers $k_{0}$ and $k_{1}$ is defined to be a sum over the admissible discrete values:

$$
\begin{aligned}
\int f\left(k_{0}, k_{1}\right) d k_{0} d k_{1} & \equiv \frac{4 \pi^{2}}{L_{0} L_{1}} \int f\left(2 \pi n_{0} / L_{0}, 2 \pi n_{1} / L_{1}\right) d n_{0} d n_{1} \\
& \equiv \frac{4 \pi^{2}}{L_{0} L_{1}} \sum_{n_{0} n_{1}} f\left(2 \pi n_{0} / L_{0}, 2 \pi n_{1} / L_{1}\right) .
\end{aligned}
$$

The factor $L_{0} L_{1} / 4 \pi^{2}$ is called the density of states because it is the number of admissible wavenumber values per unit area in the $k_{0} k_{1}$-plane. Density of states is a standard concept from thermodynamics.

The wave vectors $\left(k_{0}, k_{1}\right)$ and $\left(-k_{0},-k_{1}\right)$ describe the same physical state. To see this, note that the values of $u_{0}, u_{1}$, and $u_{2}$ are unchanged if we make the replacements $k_{0} \rightarrow-k_{0}$ and $k_{1} \rightarrow-k_{1}$, provided that we take $F\left(-k_{0},-k_{1}\right)=F\left(k_{0}, k_{1}\right)$ and $\Phi\left(-k_{0},-k_{1}\right)=-\Phi\left(k_{0}, k_{1}\right)$. Therefore, when integrating over wavenumber space, the domain of integration includes only one-half of the $k_{0} k_{1}$-plane, chosen so that only one member of each pair, $\left(k_{0}, k_{1}\right)$ and $\left(-k_{0},-k_{1}\right)$, lies in the domain of integration. Also, the domain of integration excludes the zero wave vector $\left(k_{0}, k_{1}\right)=(0,0)$, because the zero wave vector contributes zero energy and zero stress.

If the value of a function $f\left(k_{0}, k_{1}\right)$ does not depend on the direction of the wave vector, then $f$ is defined to be isotropic. In this case, we can write $f\left(k_{0}, k_{1}\right)=f(k)$, and we have

$$
\int f\left(k_{0}, k_{1}\right) d k_{0} d k_{1}=\pi \int_{0}^{\infty} f(k) k d k
$$

The coefficient on the right-hand side is $\pi$, rather than $2 \pi$, because we only integrate over onehalf of the $k_{0} k_{1}$-plane.

Property 8 . The shear stress on the fault is parallel to the $x_{1}$ direction, that is, it is oriented along-strike. The shear stress is:

$$
\tau\left(k_{0}, k_{1}\right)=-\sqrt{\frac{2 \mu E\left(k_{0}, k_{1}\right) k}{L_{0} L_{1}}} \cos \left(k_{0} x_{0}+k_{1} x_{1}+\Phi\left(k_{0}, k_{1}\right)\right) \operatorname{sgn}\left(F\left(k_{0}, k_{1}\right)\right) .
$$


The above formula shows that the set of basis displacement fields is complete in the following sense: Given any pattern of shear stress oriented along-strike, there is a linear combination of basis displacement fields which produces that pattern of shear stress. (This statement assumes that the pattern of shear stress is square-integrable, that is, the pattern of shear stress has a finite mean-square value.) In fact, the linear combination of basis displacement fields is effectively a Fourier transform of the shear stress pattern.

Property 9. The mean-square shear stress is:

$$
\tau_{\mathrm{rms}}^{2}\left(k_{0}, k_{1}\right)=\frac{\mu}{L_{0} L_{1}} E\left(k_{0}, k_{1}\right) k
$$

This equation is just the mean-square of the previous equation. The mean-square shear stress, $\tau_{\mathrm{rms}}^{2}\left(k_{0}, k_{1}\right)$, is called the power spectral density of the stress pattern.

Property 10. If you form a linear combination of these displacement fields, with different wave vectors, then the mean-square shear stress, $\tau_{\text {total }}^{2}$, of the linear combination equals the sum of the mean-square shear stresses of the individual fields:

$$
\tau_{\text {total }}^{2}=\frac{L_{0} L_{1}}{4 \pi^{2}} \int \tau_{\mathrm{rms}}^{2}\left(k_{0}, k_{1}\right) d k_{0} d k_{1}=\frac{\mu}{4 \pi^{2}} \int E\left(k_{0}, k_{1}\right) k d k_{0} d k_{1}
$$

This property is just a statement of Parseval's theorem.

\section{Thermodynamic Model Construction}

We now show how to use the basis displacement fields to construct a thermodynamic fault model.

A micro-state of the thermodynamic system is a pattern of slip on the fault surface, and the corresponding displacement of the surrounding elastic medium, under the constraint that the shear stress must be oriented along-strike. We describe a micro-state as a linear combination of the basis displacement fields. In other words, we specify a micro-state of the system by giving the amplitude, $F\left(k_{0}, k_{1}\right)$, and the phase, $\Phi\left(k_{0}, k_{1}\right)$, for each wave vector, $\left(k_{0}, k_{1}\right)$.

We noted earlier that the wave vectors $\left(k_{0}, k_{1}\right)$ and $\left(-k_{0},-k_{1}\right)$ describe the same physical state. So, when we form a linear combination of the basis displacement fields, we only include one member from each such pair of wave vectors. We also exclude the zero wave vector $\left(k_{0}, k_{1}\right)=(0,0)$ because it describes a state with zero energy and zero stress.

Because each displacement field contains both an amplitude, $F$, and a phase, $\Phi$, it represents two degrees of freedom in the thermodynamic system. This can be seen by considering the trigonometric identity

$$
F \cos \left(k_{0} x_{0}+k_{1} x_{1}+\Phi\right)=F_{C} \cos \left(k_{0} x_{0}+k_{1} x_{1}\right)+F_{S} \sin \left(k_{0} x_{0}+k_{1} x_{1}\right)
$$

where $F_{C}=F \cos \Phi$ and $F_{S}=-F \sin \Phi$. This shows that each basis displacement field can be expressed as a linear combination of two displacement fields with arbitrary amplitudes $F_{C}$ and $F_{S}$, which means that there are two degrees of freedom.

We now show that the equipartition theorem applies to our system. Recall the four premises of the equipartition theorem: 
1. The system can be decomposed into a set of degrees of freedom.

2. The total energy of the system is equal to the sum of the energies associated with each degree of freedom.

3. Each degree of freedom is a continuous real variable which can assume an arbitrary value.

4. The energy of each degree of freedom depends quadratically on its value.

Premise 1 is satisfied because a micro-state is described by a sum of basis displacement fields. The completeness property of the basis displacement fields (property 8) states that any possible pattern of fault slip oriented along-strike can be produced by some linear combination of the basis displacement fields. This shows that our basis displacement fields provide a complete description that includes every possible micro-state.

Premise 2 is satisfied because of the orthogonality property of the basis displacement fields (property 7).

Premise 3 is satisfied because the amplitude, $F$, of a basis displacement field can assume any real value, and the phase $\Phi$ can assume any value from 0 to $2 \pi$. (This is equivalent to saying that the amplitudes $F_{C}$ and $F_{S}$ mentioned in the equation above can each assume any real value.)

Premise 4 is satisfied because the energy of a basis displacement field (property 6) is proportional to $F^{2}$. (This is equivalent to saying that the two displacement fields on the righthand side of the equation above contribute energy proportional to $F_{C}^{2}$ and $F_{S}^{2}$ respectively, because $F^{2}=F_{C}^{2}+F_{S}^{2}$.)

The first conclusion of the equipartition theorem says that the Boltzmann distribution applies separately to each degree of freedom, as if the degrees of freedom are independent random variables. We use this in the following way. Our goal is to randomly generate a pattern of shear stress on the fault surface, by selecting a micro-state chosen at random according to the Boltzmann probability distribution. The equipartition theorem states that we can make the random selection by independently choosing an amplitude, $F\left(k_{0}, k_{1}\right)$, and phase, $\Phi\left(k_{0}, k_{1}\right)$, for each wave vector, $\left(k_{0}, k_{1}\right)$, according to the Boltzmann distribution. The micro-state is the sum of the resulting basis displacement fields, and we can then compute the pattern of shear stress using the stress formula (property 8) and energy formula (property 6).

The ability to select amplitudes and phases independently for each wave vector is critical, because it makes our method computationally feasible.

(Remark: In the computer implementation of the method, we randomly choose an energy, $E\left(k_{0}, k_{1}\right)$, and phase, $\Phi\left(k_{0}, k_{1}\right)$, for each wave vector; it is not necessary to compute the amplitude, $F\left(k_{0}, k_{1}\right)$.)

The second conclusion of the equipartition theorem says that each degree of freedom has the same expected energy of $1 / 2 \beta$, where $\beta$ is the thermodynamic parameter that plays the role of reciprocal temperature. Because each of our basis displacement fields represents two degrees of freedom, each basis displacement field has expected energy equal to $1 / \beta$. That is,

$$
\left\langle E\left(k_{0}, k_{1}\right)\right\rangle=\frac{1}{\beta} \quad \text { for all } k_{0}, k_{1}
$$

where the angle brackets indicated expected value.

This is a problem. To see why, use property 10 to write the expected value of the meansquare shear stress: 


$$
\left\langle\tau_{\text {total }}^{2}\right\rangle=\frac{\mu}{4 \pi^{2}} \int\left\langle E\left(k_{0}, k_{1}\right)\right\rangle k d k_{0} d k_{1}
$$

If $\left\langle E\left(k_{0}, k_{1}\right)\right\rangle$ is a constant, then the integral is strongly divergent, which means that the meansquare shear stress is infinite. So, the Boltzmann distribution predicts an infinite amount of stress, which is clearly not acceptable.

This is called an ultraviolet divergence because the divergence occurs in the limit of large $k$ or short wavelength. To proceed, we must find a way to resolve the ultraviolet divergence. 


\section{Ultraviolet Divergence}

The ultraviolet divergence that we encountered is not a new problem. In some prior methods for generating heterogeneous initial stress conditions, the power spectral density behaves asymptotically like a power of the wavenumber $k$ in the limit of large $k$ :

$$
\tau_{\mathrm{rms}}^{2}\left(k_{0}, k_{1}\right) \sim \frac{1}{k^{\alpha}} \quad \text { as } k \rightarrow \infty
$$

Any such method has an ultraviolet divergence if $\alpha \leq 2$, because in that case $1 / k^{\alpha}$ falls off so slowly that it is not integrable over the $k_{0} k_{1}$-plane. For example, Andrews and Barall (2011) describe a method with $\alpha=2$. They resolve the divergence simply by imposing a highfrequency cutoff, limiting $k$ to a maximum value chosen so that the shortest wavelengths considered are several times the resolution of the dynamic rupture simulation code.

We consider an arbitrary high-frequency cutoff to be incompatible with our thermodynamic method. Such a cutoff is not motivated by any thermodynamic principle, results in a stress state that is not well-defined independently of the discretization, and as a practical matter places too much of the stress variability into the shortest allowed wavelengths (that is, a large fraction of the power in the stress variation is concentrated in wavelengths close to the smallest wavelengths that can be resolved by the simulation code). So, we seek to resolve the ultraviolet divergence without the imposition of a cutoff.

In this section, we present a well-known example of a thermodynamic system that exhibits an ultraviolet divergence-blackbody radiation. We show that the divergence can be resolved by restricting the set of micro-states of the system. Then, we show in a general way how the same concept can be applied to an earthquake fault, with the finite strength of the fault acting to restrict the set of micro-states.

\section{Blackbody Radiation}

The term ultraviolet divergence (sometimes called ultraviolet catastrophe) gets its name from a problem in statistical mechanics: computing the spectrum of blackbody radiation.

The setting is a container which is maintained at a temperature, $T$ (fig. 4). A small viewport allows the experimenter to observe the electromagnetic radiation inside the container. The problem is to calculate the amount of electromagnetic energy at each wavelength, and the total amount of electromagnetic energy.

A micro-state of the electromagnetic field can be described as a linear superposition of plane waves. A plane wave is characterized by a wave vector, polarization, and amplitude. The total energy of the electromagnetic field equals the sum of the energies of the individual plane waves. It can be shown that the electromagnetic field has two degrees of freedom for each wave vector and polarization (for example, Reif, 1965). So according to the equipartition theorem, the expected energy in the plane wave with wave vector $\left(k_{0}, k_{1}, k_{2}\right)$ and polarization $\xi= \pm 1$ is:

$$
\left\langle E\left(k_{0}, k_{1}, k_{2}, \xi\right)\right\rangle=\frac{1}{\beta} \text {. }
$$

This immediately implies an ultraviolet divergence. Because there are an infinite number of admissible wave vectors, the equipartition theorem predicts that the total electromagnetic 
energy is infinite. Also, because the number of admissible wave vectors increases as the wavelength decreases, it predicts that the energy increases without limit as the wavelength decreases.

The ultraviolet divergence is resolved by introducing quantum mechanics, as shown by Planck. The key idea is to reduce the set of accessible micro-states. Instead of allowing the energy to have an arbitrary real value, quantum mechanics restricts the energy to a set of discrete values, called quantum levels:

$$
E\left(k_{0}, k_{1}, k_{2}, \xi\right)=n \hbar c k
$$

where $n$ is an integer, $\hbar$ is Planck's constant, $c$ is the speed of light, and $k$ is the scalar wavenumber. (Another way of saying this is that each plane wave contains an integral number of photons, where each photon carries energy, $\hbar c k$.)

It is important to note that the probability of a micro-state is still given by the Boltzmann probability distribution, even when quantum mechanics is introduced. The difference is that now the Boltzmann probability distribution is applied over a much smaller set of micro-states. With this change, the expected energy is given by the Planck distribution:

$$
\left\langle E\left(k_{0}, k_{1}, k_{2}, \xi\right)\right\rangle=\frac{1}{\beta} \frac{\beta \hbar c k}{e^{\beta \hbar c k}-1} .
$$

This resolves the ultraviolet divergence because the expression on the right-hand side has a finite sum, so the total energy is finite.

Let us look more closely at the expression $\beta \hbar c k /\left(e^{\beta \hbar c k}-1\right)$ appearing on the righthand side. For small $k$ or long wavelength, it approaches 1 , which means that the expected energy is close to classical value $1 / \beta$. But for large $k$ or short wavelength, it approaches 0 , which means that the expected energy declines to zero.

\section{Finite Fault Strength Restricts the Set of Accessible States}

Our objective is to resolve the ultraviolet divergence for earthquake faults in a manner analogous to the way that quantum mechanics does for blackbody radiation. In particular, we want to reduce the set of micro-states and obtain an expression for expected energy that is analogous to the Planck distribution. But first we must ask: What physical process acts to restrict the set of accessible micro-states on an earthquake fault?

The answer is that the finite strength of the fault restricts the set of micro-states. When we defined our basis displacement fields, we allowed the amplitudes to be arbitrarily large, which means that the shear stress on the fault can be arbitrarily large. But this is not possible, because the fault has finite strength. A micro-state cannot have shear stress that exceeds the fault's yield stress, because an earthquake would occur before such a state could be attained.

Ideally, we would require that the shear stress be less than the yield stress at every point on the fault, but such a requirement is not mathematically tractable. This is because the mathematical properties of the Fourier transform, such as Parseval's theorem, tell us about meansquare values rather than absolute maximum values. So instead, we require that the expected mean-square shear stress $\left\langle\tau_{\text {total }}^{2}\right\rangle$ be less than some fixed, finite value that depends on the strength of the fault.

Recall equation 25 that relates expected mean-square shear stress to expected energy: 


$$
\left\langle\tau_{\text {total }}^{2}\right\rangle=\frac{\mu}{4 \pi^{2}} \int\left\langle E\left(k_{0}, k_{1}\right)\right\rangle k d k_{0} d k_{1} .
$$

Now we consider the value of $\left\langle\tau_{\text {total }}^{2}\right\rangle$ to be fixed, and explore qualitatively the consequences for the expected energy $\left\langle E\left(k_{0}, k_{1}\right)\right\rangle$. For small $k$, the factor of $k$ in the integrand means that $\left\langle E\left(k_{0}, k_{1}\right)\right\rangle$ contributes very little to the stress. In other words, small- $k$ states do not "feel" the finite strength of the fault very much. So we expect that $\left\langle E\left(k_{0}, k_{1}\right)\right\rangle$ remains close to the equipartition value $1 / \beta$.

For large $k$, the factor of $k$ in the integrand means that $\left\langle E\left(k_{0}, k_{1}\right)\right\rangle$ contributes a lot to the stress. In other words, large- $k$ states do "feel" the finite strength of the fault substantially. So we expect that $\left\langle E\left(k_{0}, k_{1}\right)\right\rangle$ is substantially less than the equipartition value $1 / \beta$. In fact, $\left\langle E\left(k_{0}, k_{1}\right)\right\rangle$ must approach zero as $k \rightarrow \infty$ quickly enough so that the integral remains finite.

Finally, we note that the factor $k$ is isotropic, that is, it does not depend on the direction of the wave vector. So, we expect that the finite strength of the fault affects the value of $\left\langle E\left(k_{0}, k_{1}\right)\right\rangle$ in an isotropic manner.

To make this explicit, we introduce an energy rolloff function $g(k)$ which describes how the energy decreases with increasing wavenumber $k$ (fig. 5). It is defined by:

$$
\begin{gathered}
\left\langle E\left(k_{0}, k_{1}\right)\right\rangle=\frac{1}{\beta} g(k) \\
g(0)=1 \\
0<g(k) \leq 1 \\
\left\langle\tau_{\text {total }}^{2}\right\rangle=\frac{\mu}{4 \pi \beta} \int g(k) k^{2} d k<\infty .
\end{gathered}
$$

This is clearly analogous to the Planck distribution. The expected energy $\left\langle E\left(k_{0}, k_{1}\right)\right\rangle$ is close to $1 / \beta$ when $k \approx 0$, but approaches zero when $k \rightarrow \infty$.

Up until now, everything we did was scale-invariant, that is, there was no preferred length scale. But now, for the integral of $g(k) k^{2}$ to be finite, $g(k)$ has to approach zero over some characteristic length, $\Lambda$. We call this the correlation length. To make the definition precise, we define the correlation length to be $\Lambda=2 \pi / \hat{k}$, where $\hat{k}$ is chosen to maximize the value of $g(\hat{k}) \hat{k}^{2}$. (Note that this definition may not agree with use of the term "correlation length" as it appears elsewhere in the literature.)

So we have shown the following: If the fault has finite strength, then there must be a length scale in the system, which is the correlation length.

The integral $\int g(k) k^{2} d k$ must be proportional to $\Lambda^{-3}$, because the integrand is significant only when $k$ is less than some multiple of $1 / \Lambda$, and $g(k)$ is of order unity. So, if we regard $\left\langle\tau_{\text {total }}^{2}\right\rangle$ as being fixed by the strength of the fault, the last equation above implies a relation between the thermodynamic parameter $\beta$ and the correlation length:

$$
\beta \propto \Lambda^{-3} .
$$


We see from this relation that choosing a value for $\beta$ is equivalent to choosing a correlation length.

Note that the energy rolloff function is isotropic (that is, we write $g(k)$ and not $\left.g\left(k_{0}, k_{1}\right)\right)$ because we expect the finite fault strength to have an isotropic effect as mentioned earlier. It then follows that the expected power spectral density, $\left\langle\tau_{\mathrm{rms}}^{2}\left(k_{0}, k_{1}\right)\right\rangle$, is also isotropic:

$$
\left\langle\tau_{\mathrm{rms}}^{2}\left(k_{0}, k_{1}\right)\right\rangle=\frac{\mu}{L_{0} L_{1}}\left\langle E\left(k_{0}, k_{1}\right)\right\rangle k=\frac{\mu}{L_{0} L_{1} \beta} g(k) k
$$

Now we need to derive a formula for $g(k)$. Because we know that $g(0)=1$, the main task is to find a formula for the asymptotic behavior of $g(k)$ as $k \rightarrow \infty$. 


\section{Scaling Relations and Self-Similarity}

We derive the asymptotic behavior of the energy rolloff function $g(k)$ from a set of earthquake scaling relations. First, we state four scaling relations, and obtain a formula for the total area of stress perturbations of various sizes. Then, we can calculate the asymptotic behavior of $g(k)$ by requiring it to be consistent with the area formula.

A key assumption is that earthquakes are self-similar or scale-invariant. Self-similarity is supported by observations such as the independence of stress drop and earthquake size, and the fractal geometry of faults. It is sometimes stated that if earthquakes are self-similar, then the stress distribution ought to be self-similar too. But a self-similar stress distribution implies a $1 / k^{2}$ power spectral density, which, as we have noted, leads to infinitely large stresses (Andrews and Barall, 2011). Here we argue that, even though earthquakes are self-similar, the stress distribution is not self-similar. This deviation arises from the fact that earthquakes of different sizes repeat at different rates.

\section{Scaling Relations}

We begin by following Hanks $(1977,1979)$ who states three empirical scaling relations and then derives a formula for the rate of occurrence of earthquakes as a function of rupture size.

Scaling relation 1 is the Gutenberg-Richter relation (Gutenberg and Richter, 1954). Let $M$ be an earthquake magnitude, and let $N$ be the rate of occurrence of earthquakes with magnitude $\geq M$. Then

$$
\log _{10} N=a-M
$$

where $a$ is some constant.

Scaling relation 2 is the relation between magnitude and seismic moment (Hanks and Kanamori, 1979). Let $M_{0}$ be seismic moment, which is defined as

$$
M_{0} \propto \delta r^{2}
$$

where $\delta$ is fault slip, and $r$ is the radius of the fault rupture area. Then

$$
\log _{10} M_{0}=1.5 M+d
$$

where $d$ is some constant. Notice that the implied constant of proportionality in the definition of $M_{0}$ can be absorbed into the constant $d$.

Scaling relation 3 is the statement that earthquakes are self-similar or scale-invariant. It is

$$
\delta \propto r
$$

If rock behaves like a linear elastic material, then stress drop $\Delta \tau$ is proportional to $\delta / r$. So an equivalent way to state the assumption of self-similarity is that stress drop is independent of earthquake size, which we write as

$$
\Delta \tau \propto r^{0}
$$


Algebraically combining the above three scaling relations (see appendix C) shows that the rate of occurrence of earthquakes with radius between $r$ and $r+d r$ is:

$$
N_{\mathrm{EQ}}(r) d r=-\frac{d N}{d r} d r \propto r^{-3} d r
$$

Hanks suggests that in the distribution of shear stresses, the number of positive stress perturbations of radius $r$ should be proportional to $N_{\mathrm{EQ}}(r)$, on the basis of the following conceptual model. "[I]magine a planar fault surface large in comparison to any earthquake source dimension of interest, and a population of incipient earthquakes to occur upon it; the earthquake population is characterized by the frequency of occurrence $\left[N_{\mathrm{EQ}}(r)\right] \ldots$ Before any of the earthquakes occur, all of the stress differences that will be realized at the time of occurrence for each and every event exist on the fault surface in 'potential' form" (Hanks, 1979).

There is a problem with this interpretation of $N_{\mathrm{EQ}}(r)$. Suppose we try to calculate the total area of all the stress perturbations with radius $\leq R$ :

$$
A(r \leq R)=\int_{0}^{R} N_{\mathrm{EQ}}(r) \pi r^{2} d r \propto \int_{0}^{R} r^{-1} d r=\infty
$$

The integral diverges. The divergence tells us that there isn't enough room on the fault surface to hold all the small stress perturbations if they follow Gutenberg-Richter statistics. This is yet another example of an ultraviolet divergence because the divergence occurs at small scales.

The problem arises because Gutenberg-Richter gives the rate $N_{\mathrm{EQ}}(r)$ at which earthquakes occur over an interval of time, while the stress perturbations exist simultaneously at an instant of time, so it's incorrect to equate the two.

To find the correct statistics, we must take account of the fact that earthquakes repeat, and smaller earthquakes repeat more frequently. Because earthquakes of different sizes repeat at different rates, the stress perturbations existing at an instant of time will have statistics that differ from Gutenberg-Richter. So we introduce one more scaling relation.

Scaling relation 4 is the statement that slip deficit accumulates at a constant rate. It is

$$
T \propto \delta
$$

where $T$ is the amount of time it takes for an earthquake to repeat. This relation expresses the idea that all points on the fault must have the same average slip rate.

The number of earthquakes that occur over an interval of time is proportional to $1 / T$. So we can write

$$
N_{\mathrm{EQ}}(r) d r=\frac{1}{T} N_{\mathrm{SP}}(r) d r
$$

where $N_{\mathrm{SP}}(r) d r$ is the number of stress perturbations with radius between $r$ and $r+d r$ that exist at an instant of time. Combining this with the formula for $N_{\mathrm{EQ}}(r)$ and the scaling relations $T \propto \delta \propto r$, we get 


$$
N_{\mathrm{SP}}(r) d r=T N_{\mathrm{EQ}}(r) d r \propto r^{-2} d r .
$$

Then the total area of all stress perturbations with radius $\leq R$ is:

$$
A(r \leq R) \propto \int_{0}^{R} N_{\mathrm{SP}}(r) r^{2} d r \propto \int_{0}^{R} d r=R .
$$

By taking earthquake repetition into account, we have resolved the ultraviolet divergence.

\section{Energy Rolloff and Power Spectrum}

The scaling relation $A(r \leq R) \propto R$ tells us about the size distribution of small positive stress perturbations. Since small stress perturbations are associated with large-wavenumber or short-wavelength Fourier modes, the scaling relation also tells us something about the expected power spectral density $\left\langle\tau_{\mathrm{rms}}^{2}\left(k_{0}, k_{1}\right)\right\rangle$ of the stress field, in the limit of large wavenumber. So we ask the following question: what asymptotic behavior of the power spectral density, in the limit of large $k$, is consistent with the scaling relation?

Answering that question requires a lengthy and technical calculation, which is given in appendix D. Here we state the result. To be consistent with the scaling relation, the asymptotic behavior of the expected power spectral density is

$$
\left\langle\tau_{\mathrm{rms}}^{2}\left(k_{0}, k_{1}\right)\right\rangle \propto \frac{1}{k^{2}(\log (\zeta k))^{2}} \quad \text { as } k \rightarrow \infty .
$$

The corresponding asymptotic form of the energy rolloff function is

$$
g(k) \propto \frac{1}{k^{3}(\log (\zeta k))^{2}} \quad \text { as } k \rightarrow \infty .
$$

The parameter $\zeta$ has dimensions of length, and is a length scale related to the correlation length. The occurrence of a length scale should not be surprising, because we have previously shown that the finite strength of the fault implies the existence of a length scale.

It should be noted that to derive asymptotic formulas for the power spectral density and energy rolloff, one must make additional assumptions about the statistical properties of the stress field. For the formulas above, we assumed that the high-wavenumber part of the stress field has Gaussian statistics. The formulas can change if different assumptions are made. This is explored in appendix D.

The power spectral density differs from the $1 / k^{2}$ that would be obtained by assuming the stress itself is self-similar, although the difference is the slowly growing factor $(\log (\zeta k))^{2}$. The formula resolves our ultraviolet divergence, because for any $a>0$,

$$
\int_{a}^{\infty} \frac{1}{k^{2}(\log (\zeta k))^{2}} k d k=\frac{1}{\log (\zeta a)}<\infty .
$$

So the high- $k$ basis displacement fields no longer contribute an infinite amount of stress. 


\section{State Restriction and Probability Distribution}

Our plan for resolving the ultraviolet divergence is to restrict the set of accessible microstates, so that the expected mean-square stress is finite. This requires that the expected energy rolls off to zero as the wavenumber becomes large. In the previous section, we found the asymptotic behavior of the energy rolloff. In this section, we complete the plan by showing how to restrict the set of states and generate random values in accordance with the resulting probability distribution.

\section{Energy Rolloff Function}

In the last section we found the asymptotic behavior of the energy rolloff function, $g(k)$, in the limit of large $k$, and we also know that $g(0)=1$. We need to choose a functional form for $g(k)$ that has this behavior. This could be done in any number of ways, but we have chosen the following form because it is among the simplest formulas with correct asymptotic behavior:

$$
g(k)=\frac{1}{1+(\eta k)^{3}(\log (1+\zeta k))^{2}} .
$$

The constants $\eta$ and $\zeta$ are adjustable parameters with dimensions of length. Generally, we take $\eta=\zeta$, and adjust $\zeta$ to obtain the desired correlation length. Appendix E contains a recommended numerical algorithm. Recall that the correlation length is defined to be $\Lambda=2 \pi / \hat{k}$, where $\hat{k}$ is chosen to maximize the value of $g(\hat{k}) \hat{k}^{2}$.

In addition to describing the decrease in energy with increasing wavenumber, the energy rolloff function also establishes a relation between the thermodynamic parameter $\beta$ and the expected mean-square stress $\left\langle\tau_{\text {total }}^{2}\right\rangle$. Given one, the other can be calculated using

$$
\left\langle\tau_{\text {total }}^{2}\right\rangle=\frac{\mu}{4 \pi \beta} \int_{0}^{\infty} g(k) k^{2} d k
$$

Appendix E contains a recommended numerical algorithm for evaluating the improper integral.

\section{State Restriction}

For each wave vector $\left(k_{0}, k_{1}\right)$, we impose a maximum allowed amplitude $F_{\max }\left(k_{0}, k_{1}\right)$ for the basis displacement field:

$$
0 \leq F\left(k_{0}, k_{1}\right) \leq F_{\max }\left(k_{0}, k_{1}\right) .
$$

Because energy is proportional to the square of the amplitude, the restriction can equivalently be described as imposing a maximum allowed energy $E_{\max }\left(k_{0}, k_{1}\right)$ for the basis displacement field:

$$
0 \leq E\left(k_{0}, k_{1}\right) \leq E_{\max }\left(k_{0}, k_{1}\right) .
$$

Subject to this restriction, the amplitude $F\left(k_{0}, k_{1}\right)$ and phase $\Phi\left(k_{0} . k_{1}\right)$ are chosen randomly according to the Boltzmann probability distribution for a system with two degrees of 
freedom. Each $F_{\max }\left(k_{0}, k_{1}\right)$ or $E_{\max }\left(k_{0}, k_{1}\right)$ is chosen in such a way so that the expected energy satisfies $\left\langle E\left(k_{0}, k_{1}\right)\right\rangle=g(k) / \beta$. It can be shown that this condition is satisfied if we define

$$
E_{\max }\left(k_{0}, k_{1}\right) \equiv-\frac{1}{\beta} \log (1-H)
$$

where $H$ is the solution to the equation

$$
g(k)=1+\frac{1}{H}(1-H) \log (1-H) .
$$

Furthermore, if $U$ is a random variable uniformly distributed between 0 and 1, then

$$
E\left(k_{0}, k_{1}\right)=-\frac{1}{\beta} \log (1-H U)
$$

is randomly distributed according to the Boltzmann distribution for a system with two degrees of freedom, obeys the restriction $E\left(k_{0}, k_{1}\right) \leq E_{\max }\left(k_{0}, k_{1}\right)$, and has the expected energy $\left\langle E\left(k_{0}, k_{1}\right)\right\rangle=g(k) / \beta$. Appendix $E$ contains proofs of these formulas, plus a recommended numerical algorithm for computing $H$. This provides a practical method for calculating random energy values that obey the Boltzmann distribution, our state restriction, and our energy rolloff. 


\section{Algorithm to Generate the Stress Field}

In this section we present the algorithm for generating a random stress field. It is the sum of a set of plane waves, each associated with a basis displacement field. Amplitudes and phases are randomly selected, independently for each wave vector, according to the Boltzmann probability distribution, with the amplitudes restricted to conform with the energy rolloff function.

The stress field produced by this algorithm is a "raw" field, which generally must be post-processed before being used in a dynamic rupture simulation. Later sections will discuss post-processing operations, which typically include the following: limiting the extent of the simulated rupture; scaling the stress field to conform to the assumed fault friction; and filtering the stress field to suppress high-frequency components that cannot be resolved by the dynamic rupture simulation code. For models where the initial normal stress varies over the fault surface, the "raw" values must be scaled proportionately to the normal stress; thus, the "raw" values should be regarded as being in "friction coefficient space."

The algorithm consists of three parts. First, there are several preliminary steps. Second, in the main part of the algorithm, a set of random amplitudes and phases are generated. Third, the corresponding stress fields are summed to produce the final result.

\section{Preliminary Steps}

Step 1. Choose a correlation length, $\Lambda$. The next section will provide guidance in choosing the correlation length. The value of $\Lambda$ is typically about equal to the diameter of the earthquake rupture.

Step 2. Solve for the parameters $\eta$ and $\zeta$ that appear in the energy rolloff function $g(k)$, as described in the previous section. Typically we choose $\eta=\zeta$.

Step 3. Choose a value for the thermodynamic parameter $\beta$. If the stress field is going to be scaled during post-processing, then $\beta$ may be chosen arbitrarily because it plays the role of a scale factor which is adjusted during post-processing. Otherwise, $\beta$ could be chosen by assuming a value for the mean-square stress and using the relation between $\beta$ and mean-square stress given in the previous section.

Step 4. Choose the size of the "raw" fault surface, which is $L_{0}$ vertically and $L_{1}$ alongstrike. For reasons explained in the next section, the "raw" fault surface is typically much larger than the final fault surface used in a dynamic rupture simulation. Typically we choose $L_{0}=L_{1}$.

Step 5. Lay out a grid of points on the fault surface, which are the locations where the stress field is computed. Let $N_{0}$ and $N_{1}$ be the number of grid points vertically and along-strike, respectively. We require that $N_{0}$ and $N_{1}$ be powers of 2 (so that we can use the simplest form of the Fast Fourier Transform), and typically we choose $N_{0}=N_{1}$. The grid points $\left(x_{0}, x_{1}\right)$ are

$$
\begin{array}{ll}
x_{0}=\left(m_{0} / N_{0}\right) L_{0} & \text { for } 0 \leq m_{0}<N_{0} \\
x_{1}=\left(m_{1} / N_{1}\right) L_{1} & \text { for } 0 \leq m_{1}<N_{1}
\end{array}
$$

where $m_{0}$ and $m_{1}$ are integers. The grid is considered to satisfy periodic boundary conditions. Corresponding to this grid of points, there is a grid of wave vectors $\left(k_{0}, k_{1}\right)$ which is

$$
k_{0}=2 \pi n_{0} / L_{0} \quad \text { for }-N_{0} / 2<n_{0} \leq N_{0} / 2
$$




$$
k_{1}=2 \pi n_{1} / L_{1} \quad \text { for }-N_{1} / 2<n_{1} \leq N_{1} / 2
$$

where $n_{0}$ and $n_{1}$ are integers. The Nyquist wavenumbers are defined to be the highest wavenumbers in the grid,

$$
\begin{aligned}
& k_{0}^{*} \equiv \pi N_{0} / L_{0} \\
& k_{1}^{*} \equiv \pi N_{1} / L_{1} .
\end{aligned}
$$

Step 6. Select a set of "active" wave vectors $\left(k_{0}, k_{1}\right)$ as follows. For each pair of wave vectors $\left(k_{0}, k_{1}\right)$ and $\left(-k_{0},-k_{1}\right)$ that satisfy the condition

$$
0<\left(k_{0} / k_{0}^{*}\right)^{2}+\left(k_{1} / k_{1}^{*}\right)^{2}<1
$$

select one member of the pair to be "active" (it doesn't matter which one is selected). We only include one member of each pair because $\left(k_{0}, k_{1}\right)$ and $\left(-k_{0},-k_{1}\right)$ correspond to the same physical state. Notice that in the typical case where $k_{0}^{*}=k_{1}^{*}$, the above condition describes a circle in the $k_{0} k_{1}$-plane. We use a circle rather than a square so that the total stress field will be isotropic; a square allows shorter wavelengths along the grid diagonals than along the grid axes. We exclude the zero wave vector because it corresponds to uniform fault slip, which produces no stress. We exclude the wave vectors $\left(k_{0}^{*}, 0\right),\left(0, k_{1}^{*}\right)$, and $\left(k_{0}^{*}, k_{1}^{*}\right)$ because they each introduce only one degree of freedom, whereas our analysis assumes two degrees of freedom per wave vector.

\section{Generating Random Amplitudes and Phases}

The following steps are performed once for each "active" wave vector $\left(k_{0}, k_{1}\right)$.

Step 7. Calculate the energy rolloff function $g(k)$.

Step 8. Numerically solve the following equation for the energy truncation value $H$ :

$$
g(k)=1+\frac{1}{H}(1-H) \log (1-H) .
$$

Step 9. Generate two independent random numbers $U$ and $V$, each of which is uniformly distributed between 0 and 1 . Calculate the energy and phase according to

$$
\begin{gathered}
E\left(k_{0}, k_{1}\right)=-\frac{1}{\beta} \log (1-H U) \\
\Phi\left(k_{0}, k_{1}\right)=2 \pi V .
\end{gathered}
$$

Note that it is not necessary to calculate the displacement field amplitude $F\left(k_{0}, k_{1}\right)$ because the stress field can be computed directly from the energy and phase. 


\section{Summing the Random Stress Fields}

Step 10. For each grid point $\left(x_{0}, x_{1}\right)$, calculate the total stress field as

$$
\tau_{\text {total }}\left(x_{0}, x_{1}\right)=-\sum_{k_{0} k_{1}} \sqrt{\frac{2 \mu E\left(k_{0}, k_{1}\right) k}{L_{0} L_{1}}} \cos \left(k_{0} x_{0}+k_{1} x_{1}+\Phi\left(k_{0}, k_{1}\right)\right)
$$

where the sum runs over all "active" wave vectors $\left(k_{0}, k_{1}\right)$. The summation can be performed efficiently using the Fast Fourier Transform. This is our final "raw" stress field.

Note that the above summation formula will be modified slightly in the next section, as part of controlling the rupture extent. 


\section{Controlling Rupture Extent}

When performing a dynamic rupture simulation, some method must be used to limit the extent of the rupture. Details of several methods can be found in the SCEC/U.S. Geological Survey dynamic rupture code verification project (Harris, Barall, Archuleta, and others, 2009; Harris, Barall, Andrews, and others, 2011). The simplest technique is to impose a strength barrier, beyond which the fault is not permitted to rupture. Aside from being an arbitrary limit, the strength barrier also brings the rupture to a "hard stop," which can generate strong reflections. Another way to limit the extent of the rupture is to modify the initial stress or the friction properties outside the desired rupture area to make rupture propagation unfavorable. If the modification is gradual, it can bring the rupture to a "soft stop." For example, Andrews and Barall (2011) show how to create low initial stresses outside the desired rupture area by modifying several of the Fourier coefficients of the stress field.

Here we introduce a new method for limiting rupture extent, which creates a "soft stop" by having low initial stresses outside the rupture area. Unlike previous methods, the change in stress arises spontaneously from the randomly generated heterogeneous initial stress field and is neither arbitrarily imposed nor created by manipulation. The idea is to generate a random stress field over a very large area, much larger than the actual fault. Then, the large area is searched to find a fault-sized subarea which, just by chance, has high stress in the center and low stress at the sides.

Figure 6 shows examples of stress distributions produced by our method. Rupture extent is limited by areas of low initial stress located left, right, and below the rupture area. High initial stress prevails within the rupture area. The shape of the rupture area is random, but its size is approximately equal to the correlation length. We will see that this provides a method for selecting the correlation length.

\section{Depth Conditioning}

Our focus here is on earthquakes large enough to rupture the full depth of the seismogenic zone. The base of the seismogenic zone is defined by a systematic change in the properties of the fault, such as a transition from brittle to ductile behavior. It is therefore appropriate to introduce a non-random method to limit rupture extent at the bottom of the fault.

Rupture extent along-dip is controlled by depth conditioning, a post-processing step that systematically reduces the shear stress with increasing depth. The shear stress is multiplied by a depth-conditioning function, $\Omega$, which varies as a function of depth:

$$
\tau\left(x_{0}, x_{1}\right) \leftarrow \tau\left(x_{0}, x_{1}\right) \Omega\left(x_{0}\right)
$$

This step is performed after the stress has been scaled and filtered. (Scaling and filtering are described later.) In the examples shown here, the depth conditioning function is taken to be piecewise linear:

$$
\Omega\left(x_{0}\right)=\left\{\begin{aligned}
1.00 & \text { for } 0 \geq x_{0} \geq-14,000 \mathrm{~m} \\
0.80+0.0001\left(x_{0}+16,000 \mathrm{~m}\right) & \text { for }-14,000 \mathrm{~m} \geq x_{0} \geq-16,000 \mathrm{~m} \\
0.10+0.0002\left(x_{0}+19,500 \mathrm{~m}\right) & \text { for }-16,000 \mathrm{~m} \geq x_{0} \geq-19,500 \mathrm{~m}
\end{aligned}\right.
$$


It is, of course, possible to make the depth conditioning function be smooth rather than piecewise linear.

\section{Selection Process}

Rupture extent along-strike is controlled by a selection process, which selects the fault surface from a randomly generated stress distribution thousands of times larger.

The selection process is illustrated in figure 7. In this example, the fault surface is 48.0 $\mathrm{km}$ along-strike and $19.5 \mathrm{~km}$ along-dip, and grid points are spaced $75 \mathrm{~m}$ apart. We generate the stress distribution using $N_{0}=N_{1}=32,768$, which implies that the large stress distribution is a square measuring $2,457.6 \mathrm{~km}$ on a side. With these choices, the area of the large stress distribution is more than 6,000 times larger than the area of the fault. We consider every possible position of the fault within the large stress distribution, and pick the position which is optimal in the sense of having high stress in the center of the fault and low stress at the sides of the fault. We then cut out the portion of the stress distribution at the optimal position, and use it as the stress distribution over the fault surface.

Remark: The value of $N_{0}$ and $N_{1}$ is determined mainly by the available computer memory. The value 32,768 allows the algorithm to be performed on a computer with 48 gigabytes of memory.

Two improvements can be made to the selection process. We described the selection process as looking for high stress in the center of the fault and low stress at the sides, but the reverse would be just as good. If we found a location with low stress in the center of the fault and high stress at the sides, we could simply change the sign of the stress, because our method of randomly generating the stress field is symmetric with respect to a change of sign. This improvement effectively doubles the area of the large stress distribution, for no extra work.

A further improvement is to allow the stress field to be complex-valued. Then, instead of allowing just a change of sign, we can allow an arbitrary change in the complex phase. (Note that changing the complex phase by 180 degrees is equivalent to a change in sign.) This works because our method of generating the stress field is symmetric with respect to a change in the complex phase, and the result is another effective doubling of the area of the large stress distribution. Our algorithm incorporates this improvement.

\section{Selection Algorithm}

We now present an algorithm for finding the location of the fault within the large stress distribution. A mathematical justification of the algorithm is given in appendix $F$.

Step 1. Create a selection template. It is a function $Q\left(x_{0}, x_{1}\right)$, which is positive in the portion of the fault surface where we hope to have high stress and negative in the portion of the fault surface where we hope to have low stress. It is zero in areas where we don't care about the stress. Our objective is to find the place in the large randomly generated stress distribution that is most similar to the selection template.

Note that $Q\left(x_{0}, x_{1}\right)$ is defined for all points $\left(x_{0}, x_{1}\right)$ throughout the large stress distribution, including points that are outside the fault surface. It is created under the assumption that the upper left corner of the fault surface is at the origin $\left(x_{0}, x_{1}\right)=(0,0)$. Generally $Q\left(x_{0}, x_{1}\right)$ would be zero outside the fault surface, although nonzero values may extend a short distance outside the fault surface to allow the selection template to taper to zero rather than have a discontinuity at the edge of the fault surface. To make the selection template sensitive to 
variations in stress rather than the absolute level of stress, it is adjusted to have zero DC component, that is,

$$
\sum_{x_{0} x_{1}} Q\left(x_{0}, x_{1}\right)=0
$$

where the sum runs over all points $\left(x_{0}, x_{1}\right)$ in the large stress distribution. An example of a selection template is shown in figure 8 . In the example, $Q\left(x_{0}, x_{1}\right)$ is positive in the center 30.0 $\mathrm{km}$ of the fault surface, because we aim to produce a rupture about $30-\mathrm{km}$ wide. It is negative on the left and right sides of the fault surface. The selection template is zero in the bottom $2.5 \mathrm{~km}$ of the fault surface, because depth conditioning forces the stress to be low there, so we don't care about the randomly generated stresses near the bottom of the fault surface.

Step 2. Calculate a complex-valued random shear stress field:

$$
\tau_{\text {total }}^{\prime}\left(x_{0}, x_{1}\right)=-\sum_{k_{0} k_{1}} \sqrt{\frac{2 \mu E\left(k_{0}, k_{1}\right) k}{L_{0} L_{1}}} \exp \left(i k_{0} x_{0}+i k_{1} x_{1}+i \Phi\left(k_{0}, k_{1}\right)\right)
$$

The randomly generated energies $E\left(k_{0}, k_{1}\right)$ and phases $\Phi\left(k_{0}, k_{1}\right)$ are the same ones described in the previous section. In fact, the previously described $\tau_{\text {total }}\left(x_{0}, x_{1}\right)$ is just the real part of $\tau_{\text {total }}^{\prime}\left(x_{0}, x_{1}\right)$. The summation can be done efficiently using the Fast Fourier Transform. field:

Step 3. Compute the convolution of the selection template and the complex-valued stress

$$
\left(Q \star \tau_{\text {total }}^{\prime}\right)\left(x_{0}, x_{1}\right) \equiv \sum_{y_{0} y_{1}} Q\left(y_{0}, y_{1}\right) \tau_{\text {total }}^{\prime}\left(x_{0}+y_{0}, x_{1}+y_{1}\right)
$$

The convolution can be computed efficiently using the Fast Fourier Transform. Because we use periodic boundary conditions on the large stress distribution, the sums $x_{0}+y_{0}$ and $x_{1}+y_{1}$ on the right-hand side are taken to wrap around cyclically. Note that convolution is usually defined using $x_{0}-y_{0}$ and $x_{1}-y_{1}$ on the right-hand side, but for our purposes it is more convenient to use the plus sign.

Step 4. Find the point $\left(\tilde{x}_{0}, \tilde{x}_{1}\right)$ in the large stress distribution that maximizes the absolute value of the convolution, $\left|\left(Q \star \tau_{\text {total }}^{\prime}\right)\left(\tilde{x}_{0}, \tilde{x}_{1}\right)\right|$. This will be the upper left corner of the fault surface within the large stress distribution.

Step 5. Let $\theta$ be the complex phase of the convolution $\left(Q \star \tau_{\text {total }}^{\prime}\right)\left(\tilde{x}_{0}, \tilde{x}_{1}\right)$ at the point where its absolute value is maximized. In other words, write

$$
\left(Q \star \tau_{\text {total }}^{\prime}\right)\left(\tilde{x}_{0}, \tilde{x}_{1}\right)=\left|\left(Q \star \tau_{\text {total }}^{\prime}\right)\left(\tilde{x}_{0}, \tilde{x}_{1}\right)\right| \cdot e^{i \theta}
$$

Then calculate the real-valued random shear stress as:

$$
\tau\left(x_{0}, x_{1}\right)=-\sum_{k_{0} k_{1}} \sqrt{\frac{2 \mu E\left(k_{0}, k_{1}\right) k}{L_{0} L_{1}}} \cos \left(k_{0} x_{0}+k_{1} x_{1}+\Phi\left(k_{0}, k_{1}\right)-\theta\right) .
$$


Note that each cosine term is phase-shifted by the same amount $\theta$.

Step 6. Place the upper left corner of the fault surface at location $\left(\tilde{x}_{0}, \tilde{x}_{1}\right)$. Cut out the part of the stress distribution $\tau\left(x_{0}, x_{1}\right)$ that lies within the fault surface, and discard the rest. The remaining $\tau\left(x_{0}, x_{1}\right)$ is the randomly generated stress field on the fault surface, subject to further post-processing.

\section{Correlation Length}

The selection process gives us a way to determine the correlation length $\Lambda$, although it requires some trial and error. If the correlation length is set too small, then it will be impossible to find a stress pattern that supplies high stress over the desired area of the rupture. As a result, it won't be possible to successfully execute a dynamic rupture simulation.

Our procedure is to select the smallest correlation length that reliably gives stress patterns that can sustain a rupture of the desired size. Empirically, it appears that the correlation length should be about equal to the desired diameter of the rupture.

Figure 9 shows the effect of varying the correlation length. It shows stress patterns generated with correlation lengths of 10,30 , and $100 \mathrm{~km}$, with a desired rupture size of $30 \mathrm{~km}$. A correlation length of $10 \mathrm{~km}$ is too small, because it is impossible to find any high-stress area large enough to sustain a rupture $30 \mathrm{~km}$ across. A correlation length of $100 \mathrm{~km}$ is larger than necessary and yields a fairly clean division of the fault into high and low stress areas. By comparison, a correlation length of $30 \mathrm{~km}$ is about right. It yields a high stress area large enough to sustain a rupture $30 \mathrm{~km}$ across, yet the edges of the high-stress area are ragged, with the high and low stress areas intruding into each other.

To carry out this procedure, we suggest generating stress distributions with several different correlation lengths, beginning with the desired rupture diameter, and then adding correlation lengths both larger and smaller by factors of two or three. Then, check which correlation lengths produce stress patterns able to sustain ruptures of the desired size. Correlation lengths that are too small can often be identified by simple visual inspection of the stress pattern, noting the absence of any high-stress area approximating the desired rupture size, as shown in the 10-km panel of figure 9. It is also possible to identify too-small correlation lengths by attempting to perform a dynamic rupture simulation on the stress pattern and noting that the rupture either cannot be nucleated, or dies out well before it reaches the desired size. The smallest correlation length that reliably leads to successful dynamic rupture simulations of the desired size is the correlation length that should be used.

\section{Application to Smaller Earthquakes}

A notable feature of the method just described is that the rupture is limited vertically and horizontally by two different techniques. The rupture is limited at the bottom by imposing depth conditioning, whereas the rupture is limited at the left and right by a selection process.

The reason for this difference is that our discussion focuses on the case where the earthquake is large enough to rupture the full depth of the seismogenic zone. In nature, large earthquake ruptures stop at the bottom of the seismogenic zone because of a change in the fault properties, specifically, a transition from brittle to ductile behavior. What causes such ruptures to stop as they propagate along-strike is not well understood, but does not appear to be the result of any major change in fault properties. Our use of different techniques in the along-dip and alongstrike directions reflects this difference in nature. 
In the case of smaller earthquakes, where the rupture does not extend all the way from the top to the bottom of the fault, it would not be appropriate to use two different techniques. In this case, depth conditioning should not be used. Instead, the selection process alone is used to limit rupture extent in all directions. The selection template $Q\left(x_{0}, x_{1}\right)$ is chosen to be positive in a region that approximates the desired rupture extent, which could be a circle, square, ellipse, or rectangle. 


\section{Post-Processing}

The previous section completed the construction of a raw random stress field on a fault surface. Before the stress field can be used as input to a dynamic rupture simulation, it is generally necessary to perform some post-processing steps. The steps required will depend on the details of the model, such as the friction parameters, the variation of normal stress with depth, and the characteristics of the simulation software. A typical post-processing sequence could include the following steps:

- Stress field rotation. If stresses are higher near the Earth's surface than in the lower part of the fault, then rotate the stress pattern 180 degrees to place the higher stresses in the lower part of the fault. This is useful because high stress at depth is usually more effective at driving the rupture.

- Filtering. Apply a low-pass spatial filter to suppress stress fluctuations that are below the resolution of the dynamic rupture simulation code.

- Scaling. Apply a scale factor and offset (that is, a linear transformation) to the stress pattern. Scaling is used to make the initial stresses consistent with the friction law. In the high-stress (center) portion of the fault surface, the stresses must be high enough to sustain the rupture but below the fault's yield stress. In the low-stress (side and bottom) portion of the fault surface, the stresses must be low enough to stop the rupture.

- Hypocenter selection. Select a point within a region of relatively high initial stress to be the hypocenter.

- Depth conditioning. As discussed earlier, stresses at the bottom of the fault are reduced, to limit the extent of the rupture along-dip.

- Scaling by normal stress. The raw stress values are multiplied by a factor proportional to the initial normal stress on the fault. This is done because, in general, friction forces are proportional to the normal stress. Another way of saying this is that we treat our randomly generated values as if they lie in "friction coefficient space."

Appendix G contains algorithms and further details for post-processing. 


\section{Nucleation}

A dynamic rupture simulation must have some way to start, or nucleate, the rupture. If the initial conditions are set up so that the initial shear stress is everywhere less than the fault's yield stress, then nothing will happen unless some artificial means of nucleating the rupture is implemented. Descriptions of several nucleation methods can be found, e.g., in the SCEC Dynamic Rupture Code Validation benchmarks (Harris, Barall, Archuleta, and others, 2009; Harris, Barall, Andrews, and others, 2011), and in Bizzarri (2010).

Here we introduce a new two-stage nucleation method. The method is designed to let the rupture respond to the heterogeneous initial stresses beginning very early in the simulation. The first stage is a small zone of forced rupture surrounding the hypocenter, which starts the rupture going. The second stage is a larger zone of reduced fracture energy, which allows the rupture to become large enough so that further expansion is possible without additional assistance.

\section{The Problem of Nucleation}

One of the challenges in performing dynamic rupture simulations is to find a way to nucleate the rupture. The problem is that, for the range of parameter values typically used in dynamic rupture simulations, the rupture does not become self-sustaining until it has a radius of several kilometers (this is discussed further below). So, one must introduce an artificial mechanism to propel the rupture forward until it becomes large enough to sustain itself.

We do not attempt to give a comprehensive treatment of nucleation techniques that have been used in the past, but refer the reader to the SCEC website (http://scecdata.usc.edu/cvws) for detailed descriptions of several techniques.

One simple technique is to establish a nucleation zone surrounding the hypocenter, with a radius of several kilometers, and to set the shear stress within the nucleation zone to be higher than the fault's yield stress. This high stress can be applied instantaneously at the beginning of the simulation or gradually over some interval of time; both variants have been used in the SCEC benchmarks. This technique has the disadvantage that the final slip in the nucleation zone is much larger than elsewhere on the fault. Also, it injects a large amount of energy into the rupture, which can make earthquake characteristics sensitive to the details of the nucleation process.

Another technique is to alter the frictional properties of the fault near the hypocenter, for example, by lowering the yield stress to below the initial shear stress. Again, this can be done either instantaneously at the start of the simulation or gradually over an interval of time. Often, the altered frictional properties are applied in an expanding circle surrounding the hypocenter, creating a forced rupture front (Andrews, 2004). This technique has the disadvantage that rupture propagation is controlled by the pre-determined nucleation process, at least until the rupture is large enough to become self-sustaining. Note that by the time the rupture becomes selfsustaining, it already fills an appreciable fraction of the fault surface.

We are interested in studying the effects of stochastically generated heterogeneous initial stresses. So, our interest is to have the rupture propagation and the slip pattern be controlled by the heterogeneous initial stresses, and not by the nucleation process, to the greatest extent possible. The new nucleation technique described here is an attempt to reduce the influence of the nucleation process on the rupture propagation and slip pattern, as compared to prior techniques. 


\section{The Day Radius}

Day (1982) gives a formula for the minimum radius $R_{D}$ that a rupture must attain, so that further expansion of the rupture is energetically favorable, that is, so that the elastic energy released by expansion of the rupture exceeds the fracture energy consumed:

$$
R_{D}=\frac{7 \pi \mu\left(\tau_{s}-\tau_{d}\right) D_{c}}{24\left(\tau-\tau_{d}\right)^{2}}
$$

Here, $\mu$ is the shear modulus, $\tau$ is the initial shear stress, $\tau_{s}$ is the static yield stress, $\tau_{d}$ is the dynamic sliding stress, and $D_{c}$ is the slip-weakening critical distance, that is, the amount of slip over which the frictional stress declines from $\tau_{s}$ to $\tau_{d}$. The fracture energy per unit area is $\left(\tau_{s}-\tau_{d}\right) D_{c} / 2$

The above formula for the Day radius was derived for the case of uniform shear stress, for a circular rupture zone, on a fault with uniform frictional properties, embedded in a uniform linear elastic fullspace. However, we have found the formula to be a useful approximation in other cases. When stresses or friction parameters vary with position on the fault, we insert into the Day formula their average values within a neighborhood of the hypocenter.

For the range of parameter values typically used in dynamic rupture simulations, the Day radius $R_{D}$ is on the order of $3 \mathrm{~km}$. The nucleation problem is that a simulated rupture does not become self-sustaining until its radius is approximately the Day radius.

Notice that $R_{D}$ is proportional to the slip-weakening critical distance $D_{c}$. If $D_{c}$ is made to vary with location on the fault surface, then $R_{D}$ and the fracture energy are also variable. In particular, if $D_{c}$ is made proportional to the distance from the hypocenter, then it is possible for the rupture radius to be approximately equal to $R_{D}$ over an extended range of rupture sizes. Note that only the value of $R_{D}$ near the rupture front is relevant to the question of whether or not it is energetically favorable for the rupture to expand, because it is only near the rupture front that fracture energy is consumed. These observations are the basis of our two-stage nucleation method.

(In principle, the slip-weakening critical distance $D_{c}$ is a physical parameter. In practice, the value of $D_{c}$ used in a dynamic rupture simulation is much larger than the critical distance observed in laboratory experiments. It is chosen large enough so that the model is able to resolve the cohesive zone, which is the area where slip has begun but not yet reached the critical distance. It is for this reason that we are free to manipulate $D_{c}$ to help achieve nucleation. Also, notice that as computers become more powerful and are able to run models with finer resolution, the practical value of the Day radius $R_{D}$ will decrease.)

\section{Two-Stage Nucleation Method}

Our two-stage method constructs two circular zones surrounding the hypocenter, a small zone of forced rupture with radius $R_{\mathrm{ZFR}}$, and a larger zone of reduced fracture energy with radius $R_{\mathrm{ZRFE}}$. We take $R_{\mathrm{ZFR}}$ equal to one-fourth of $R_{\mathrm{ZRFE}}$. The radius $R_{\mathrm{ZRFE}}$ is approximately equal to the Day radius, although it may vary somewhat depending on the model parameters, and some experimentation may be necessary to choose a value for $R_{\mathrm{ZRFE}}$ that produces reliable nucleation. For example, in the SCEC Dynamic Rupture Code Validation benchmarks TPV18 through TPV21, the radii are $R_{\mathrm{ZFR}}=900 \mathrm{~m}$ and $R_{\mathrm{ZRFE}}=3,600 \mathrm{~m}$. Appendix $\mathrm{G}$ contains additional information on selecting $R_{\mathrm{ZFR}}$ and $R_{\mathrm{ZRFE}}$. 
The first stage occurs within the zone of forced rupture. Each point within the zone is assigned a time, $T$, of forced rupture,

$$
T=\left\{\begin{aligned}
r /\left(0.7 V_{S}\right), & \text { if } r \leq 0.8 R_{\mathrm{ZFR}} \\
\left(0.8 R_{\mathrm{ZFR}}\right) /\left(0.7 V_{S}\right)+\left(r-0.8 R_{\mathrm{ZFR}}\right) /\left(0.35 V_{S}\right), & \text { if } 0.8 R_{\mathrm{ZFR}} \leq r \leq R_{\mathrm{ZFR}}
\end{aligned}\right.
$$

where $r$ is distance from the hypocenter, and $V_{S}$ is the shear-wave velocity. At the time $T$, the frictional stress drops immediately to the dynamic sliding stress. The result is a circular front of forced rupture, which expands outward from the hypocenter at a speed of $0.7 V_{S}$ when $r \leq$ $0.8 R_{\mathrm{ZFR}}$, and $0.35 V_{S}$ when $0.8 R_{\mathrm{ZFR}} \leq r \leq R_{\mathrm{ZFR}}$. Forced rupture does not occur outside the zone.

The first stage by itself is not sufficient to nucleate the earthquake, because the zone of forced rupture is much smaller than the Day radius. So we introduce a second stage within the zone of reduced fracture energy. Each point within the zone is assigned a reduced value $D_{c}^{\prime}$ of the slip-weakening critical distance,

$$
D_{c}^{\prime}=\left\{\begin{aligned}
0.1 D_{c}, & \text { if } r \leq 0.1 R_{\mathrm{ZRFE}} \\
\left(r / R_{\mathrm{ZRFE}}\right) D_{c}, & \text { if } 0.1 R_{\mathrm{ZRFE}} \leq r \leq R_{\mathrm{ZRFE}}
\end{aligned}\right.
$$

where $D_{c}$ is the value of the slip-weakening critical distance outside the zone of reduced fracture energy. Notice that $D_{c}^{\prime}$ is proportional to $r$ when $0.1 R_{\mathrm{ZRFE}} \leq r \leq R_{\mathrm{ZRFE}}$. As discussed above, the result is a variable Day radius, which permits the rupture radius to be approximately equal to the Day radius over the size range $0.1 R_{\mathrm{ZRFE}} \leq r \leq R_{\mathrm{ZRFE}}$. In other words, the radius $r$ at which rupture expansion becomes energetically favorable is reduced by approximately a factor of 10 .

Our two-stage nucleation method has been used successfully in six of the SCEC Dynamic Rupture Code Validation benchmarks. 


\section{Conclusions}

This report presents a new method for generating random heterogeneous initial stresses, for use in dynamic rupture simulations. The method employs concepts and techniques drawn from thermodynamics and statistical mechanics.

A pattern of slip on the fault surface is taken to be analogous to a micro-state of a thermodynamic system. Fault slip distorts the surrounding elastic medium, and the resulting elastic energy is taken to be the energy of the micro-state. Then, the Boltzmann probability distribution gives the probability of each possible pattern of slip and corresponding pattern of stress.

Although the idea is simple in principle, there are challenges in carrying it out. To make the method computationally feasible, the system must be decomposed into a set of degrees of freedom, so that the Boltzmann distribution can be applied independently to each degree of freedom. We decompose the system by introducing a set of three-dimensional basis displacement fields, each of which is an approximate solution to the elastostatic problem for a sinusoidal pattern of fault slip. The basis fields have the crucial property of orthogonality, which means that the energy of a linear combination of basis fields equals the sum of the energies of the individual fields. Orthogonality implies that the basis displacement fields behave like the degrees of freedom of a thermodynamic system, allowing the amplitudes of the basis fields to be selected independently according to the Boltzmann distribution.

A straightforward application of the Boltzmann distribution leads to infinitely large stresses on the fault. This phenomenon is a consequence of the equipartition theorem and is called an ultraviolet divergence because the divergence occurs in the limit of small wavelength.

The finite strength of the fault acts to restrict the possible states of the system, because a state that includes shear stresses in excess of the fault's yield stress cannot occur. When the finite fault strength is taken into account, the energy spectrum must deviate from equipartition, with the expected energy falling off to zero as the wavenumber approaches infinity. To find a formula for the energy falloff, we reconsider several earthquake scaling relations - the Gutenberg-Richter relation, the magnitude-moment relation, and earthquake self-similarity - and we introduce a new scaling relation of constant slip deficit accumulation. From these scaling relations, we are able to deduce that the expected power spectral density in the limit of large wavenumber is proportional to $1 / k^{2}(\log (\zeta k))^{2}$, where $k$ is the scalar wavenumber and $\zeta$ is a length scale related to the correlation length. With this formula in hand, the ultraviolet divergence is resolved, and we are able to construct a complete algorithm for generating random patterns of stress. It is noteworthy that the algorithm has only one free parameter, which is the correlation length.

We have also introduced a new selection method for limiting the extent of a simulated earthquake rupture. We generate a random stress pattern over an area thousands of times larger than the fault surface and then select a fault-sized portion of the stress pattern which, just by chance, has a positive stress perturbation of the desired size. The simulated rupture then stops automatically when it reaches the low-stress areas at the edge of the stress perturbation, without the need to impose hard barriers.

In addition to limiting rupture extent, the selection method also yields one-point statistics for the stress distribution that are heavy-tailed (by comparison to Gaussian). Furthermore, the selection method provides a way to determine the appropriate correlation length, because if the correlation length is made too small then it is impossible to find a positive stress perturbation of the desired size. With this observation, the number of free parameters governing the probability distribution is reduced to zero. (Of course, there remain many free parameters in the post- 
processing procedure, nucleation process, friction law, and elsewhere in the dynamic rupture simulation.)

Finally, we have introduced a new two-stage nucleation method, which combines a small zone of forced rupture with a larger zone of reduced fracture energy. The two-stage nucleation method is designed so that the rupture propagation can become responsive to the heterogeneous initial stresses beginning very early in the simulation.

All of this has been successfully tested in the Southern California Earthquake Center Dynamic Rupture Code Validation group. Ten different dynamic rupture codes performed simulations using heterogeneous initial stress patterns generated by our method. All ten codes produced results in excellent agreement with each other, demonstrating that our method is wellsuited to generating heterogeneous initial stress conditions for dynamic rupture simulations.

\section{Acknowledgments}

This work is supported by the U.S. Geological Survey Earthquake Science Center, by the Southern California Earthquake Center, and by the Pacific Gas and Electric Company. Thanks to Joe Andrews and Brad Aagaard for reviewing the manuscript. 


\section{References}

Aagaard, B.T., and Heaton, T.H., 2008, Constraining fault constitutive behavior with slip and stress heterogeneity: Journal of Geophysical Research, v. 113, B04301, 18 p., doi:10.1029/2006JB004793.

Abaimov, S.G., 2009, Critical behavior of slider-block model: arXiv:0902.3767v4 [condmat.stat-mech], available at http://arxiv.org/abs/0902.3767.

Andrews, D.J., 1974, Evaluation of static stress on a fault plane from a Green's function: Bulletin of the Seismological Society of America, v. 64, no. 6, p. 1,629-1,633.

Andrews, D.J., 1980, A stochastic fault model, 1. Static case: Journal of Geophysical Research, v. 85 , no. B7, p. 3,867-3,877, doi: 10.1029/JB085iB07p03867.

Andrews, D.J., 1981, A stochastic fault model, 2. Time-dependent case: Journal of Geophysical Research, v. 86, no. B11, p. 10,821-10,834, doi: 10.1029/JB086iB11p10821.

Andrews, D.J., 2004, Rupture models with dynamically determined breakdown displacement: Bulletin of the Seismological Society of America, v. 94, no. 3, p. 769-775, doi: 10.1785/0120030142.

Andrews, D.J., and Barall, M., 2011, Specifying initial stress for dynamic heterogeneous earthquake source models: Bulletin of the Seismological Society of America, v. 101, no. 5, p. 2,408-2,417, doi: 10.1785/0120110012.

Beroza, G.C., and Mikumo, T., 1996, Short slip duration in dynamic rupture in the presence of heterogeneous fault properties: Journal of Geophysical Research, v. 101, no. B10, p. 22,44922,460, doi: 10.1029/96JB02291.

Bizzarri, A., 2010, How to promote earthquake ruptures-Different nucleation strategies in a dynamic model with slip-weakening friction: Bulletin of the Seismological Society of America, v. 100, no. 3, p. 923-940, doi: 10.1785/0120090179.

Bizzarri, A., 2011, On the deterministic description of earthquakes: Reviews of Geophysics, v. 49, RG3002, 32 p., doi: 10.1029/2011RG000356.

Boatwright, J., 1981, Quasi-dynamic models of simple earthquakes-Application to an aftershock of the 1975 Oroville, California, earthquake: Bulletin of the Seismological Society of America, v. 71, no. 1, p. 69-94.

Boltzmann, L., 1876, Über die natur der gasmoleküle (On the nature of gas molecules): Wiener Berichte, v. 74, p. 553-560.

Bouchon, M., 1997, The state of stress on some faults of the San Andreas system as inferred from near-field strong motion data: Journal of Geophysical Research, v. 102, no. B6, p. 11,731-11,744, doi: 10.1029/97JB00623.

Das, S., and Aki, K., 1977, Fault plane with barriers-A versatile earthquake model: Journal of Geophysical Research, v. 82, no. 36, p. 5,658-5,670, doi: 10.1029/JB082i036p05658.

Day, S.M., 1982, Three-dimensional simulation of spontaneous rupture-The effect of nonuniform prestress: Bulletin of the Seismological Society of America, v. 72, no. 6, p. 1,8811,902 .

Day, S.M., Yu, G., and Wald, D., 1998, Dynamic stress changes during earthquake rupture:

Bulletin of the Seismological Society of America, v. 88, no. 2, p. 512-522.

Fefferman, C., 1971, On the convergence of multiple Fourier series: Bulletin of the Seismological Society of America, v. 77, no. 5, p. 744-745. 
Guatteri, M., Mai, P.M., and Beroza, G.C., 2004, A pseudo-dynamic approximation to dynamic rupture models for strong ground motion prediction: Bulletin of the Seismological Society of America, v. 94, no. 6, p. 2,051-2,063, doi: 10.1785/0120040037.

Guatteri, M., P. Mai, P.M., Beroza, G.C., and Boatwright, J., 2003, Strong ground-motion predictions from stochastic-dynamic source models: Bulletin of the Seismological Society of America, v. 93, no. 1, p. 301-313, doi: 10.1785/0120020006.

Gutenberg, B., and Richter, C., 1954, Seismicity of the Earth and associated phenomena (2nd ed.): Princeton University Press.

Hanks, T.C., 1977, Earthquake stress drops, ambient tectonic stresses, and stresses that drive plate motions: Pure and Applied Geophysics, v. 115, no. 1-2, p. 441-458, doi: 10.1007/BF01637120.

Hanks, T.C., 1979, $b$ Values and $\omega^{-\gamma}$ seismic source models-Implications for tectonic stress variations along active crustal fault zones and the estimation of high-frequency strong ground motion: Journal of Geophysical Research, v. 84, no. B5, p. 2,235-2,242, doi: 10.1029/JB084iB05p02235.

Hanks, T.C., and Kanamori, H., 1979, A moment magnitude scale: Journal of Geophysical Research, v. 84, no. B5, p. 2,348-2,350, doi: 10.1029/JB084iB05p02348.

Harris, R.A., 2004, Numerical simulations of large earthquakes-Dynamic rupture propagation on heterogeneous faults: Pure and Applied Geophysics, v. 161, no.11/12, p. 2,171-2,181, doi: 10.1007/s00024-004-2556-8.

Harris, R.A., Barall, M., Andrews, D.J., Duan, B., Ma, S., Dunham, E.M., Gabriel, A.-A., Kaneko, Y., Kase, Y., Aagaard, B.T., Oglesby, D.D., Ampuero, J.-P., Hanks, T.C., and Abrahamson, N., 2011, Verifying a computational method for predicting extreme ground motion: Seismological Research Letters, v. 82, no. 5, p. 638-644, doi: 10.1785/gssrl.82.5.638.

Harris, R.A., Barall, M., Archuleta, R., Dunham, E., Aagaard, B., Ampuero, J.-P., Bhat, H., Cruz-Atienza, V., Dalguer, L., Dawson, P., Day, S., Duan, B., Ely, G., Kaneko, Y., Kase, Y., Lapusta, N., Liu, Y., Ma, S., Oglesby, D., Olsen, K., Pitarka, A., Song, S., and Templeton, E., 2009, The SCEC/USGS Dynamic Earthquake Rupture Code Verification Exercise:

Seismological Research Letters, v. 80, no 1, p. 119-126, doi: 10.1785/gssrl.80.1.119.

Huang. Y., Saleur, H., Sammis, C., and Sornette, D., 1998, Precursors, aftershocks, criticality and self-organized criticality: Europhysics Letters, v. 41, no 1, p. 43-48, doi: 10.1209/epl/i1998-00113-X.

Ising, E. (1925), Beitrag zur theorie des ferromagnetismus (Contribution to the theory of ferromagnetism): Zeitschrift für Physik, v. 31, no 1, p. 253-258, doi: 10.1007/BF02980577.

Kaneko, Y., Avouac, J.-P., and Lapusta, N., 2010, Towards inferring earthquake patterns from geodetic observations of interseismic coupling: Nature Geoscience, v. 3, p. 363-369, doi: $10.1038 /$ ngeo843.

Lapusta, N., 2009, Seismology-The roller coaster of fault friction: Nature Geoscience, v. 2, p. 676-677, doi: 10.1038/ngeo645.

Lavallée, D., Liu, P., and Archuleta, R.J., 2006, Stochastic model of heterogeneity in earthquake slip spatial distributions: Geophysical Journal International, v. 165, no. 2, p. 622-640 doi: 10.1111/j.1365-246X.2006.02943.X.

Mai, P.M., and Beroza, G.C., 2002, A spatial random field model to characterize complexity in earthquake slip: Journal of Geophysical Research, v. 107, 2308, 21 p., doi: 10.1029/2001JB000588. 
Mavroeidis, G.P., and Papageorgiou, A.S., 2010, Effect of fault rupture characteristics on nearfault strong ground motions effect of fault rupture: Bulletin of the Seismological Society of America, v. 100 no. 1, p. 37-58, doi: 10.1785/0120090018.

McGarr, A., Spottiswoode, S.M., Gay, N.C., and Ortlepp, W.D., 1979, Observations relevant to seismic driving stress, stress drop, and efficiency: Journal of Geophysical Research, v. 84, no. B5, p. 2,251-2,261, doi: 10.1029/JB084iB05p02251.

Mikumo, T., and Miyatake, T., 1978, Dynamical rupture process on a three-dimensional fault with non-uniform frictions and near-field seismic waves: Geophysical Journal of the Royal Astronomical Society, v. 54, no. 2, p. 417-438, doi: 10.1111/j.1365-246X.1978.tb04267.x.

National Institute of Standards and Technology, 2011, Digital library of mathematical functions (2011-08-29): National Institute of Standards and Technology, http://dlmf.nist.gov.

Oglesby, D.D., and Day, S.M., 2002, Stochastic fault stress-Implications for fault dynamics and ground motion: Bulletin of the Seismological Society of America, v. 92, no. 8, p. 3,0063,021, doi: 10.1785/0120010249.

Onsager, L., 1944, Crystal statistics. I. A two-dimensional model with an order-disorder transition: Physical Review, v. 65, no. 3-4, p. 117-149, doi:10.1103/PhysRev.65.117.

Peyrat, S., Olsen, K., and Madariaga, R., 2001, Dynamic modeling of the 1992 Landers earthquake: Journal of Geophysical Research, v. 106, no. B11, p. 26,467-26,482, doi: 10.1029/2001JB000205.

Reif, F., 1965, Fundamentals of statistical and thermal physics: McGraw-Hill.

Ripperger, J., Ampuero, J.-P., Mai, P.M., and Giardini, D., 2007, Earthquake source characteristics from dynamic rupture with constrained stochastic fault stress: Journal of Geophysical Research, v. 112, B04311, 17 p., doi: 10.1029/2006JB004515.

Ripperger, J., Mai, P.M., and Ampuero, J.-P., 2008, Variability of near-field ground motion from dynamic earthquake rupture simulations: Bulletin of the Seismological Society of America, v. 98, no. 3, p. 1,207-1,228, doi: 10.1785/0120070076.

Royden, H., 1968, Real analysis (2nd ed.): Macmillan.

Rundle, J., Turcotte, D., Shcherbakov, R., Klein, W., and Sammis, C., 2003, Statistical physics approach to understanding the multiscale dynamics of earthquake fault systems, Reviews of Geophysics, v. 41, 1019, 30 p., doi: 10.1029/2003RG000135.

Schmedes, J., Archuleta, R.J., and Lavallée, D., 2010, Correlation of earthquake source parameters inferred from dynamic rupture simulations: Journal of Geophysical Research, v. 115, B03304, 12 p., doi: 10.1029/2009JB006689.

Shi, Z., and Day, S.M., 2011, 3D Simulations of dynamic rupture on rough faults: American Geophysical Union Fall Meeting, San Francisco, December 5-9, 2011, abstract S54C-06.

Smalley, R., Jr., Turcotte, D., and Solla, S., 1985, A renormalization group approach to the stickslip behavior of faults: Journal of Geophysical Research, v. 90, no. B2, p. 1,894-1,900, doi: 10.1029/JB090iB02p01894.

Tullis, T.E., 2007, Friction of rock at earthquake slip rates, in Kanamori, H., ed., Treatise on geophysics: Amsterdam and Boston, Elsevier, v. 4, chap. 5, p. 131-152.

Yamashita, T., 1976, On the dynamical process of fault motion in the presence of friction and inhomogeneous initial stress-Part I. Rupture propagation: Journal of Physical Earth, v. 24, no. 4, p. 417-444, doi: 10.4294/jpe1952.24.417. 


\section{Appendix A. Slider-Block Model}

In this appendix we explain why we must use a three-dimensional fault model, rather than a two-dimensional model.

We wish to construct an earthquake fault model that incorporates concepts from thermodynamics. It is natural to consider slider-block models, because these models have obvious similarities to the Ising model. (See Rundle and others, 2003; Abaimov, 2009.) In this appendix we show that a slider-block model is inappropriate for our application, because it has incorrect energy spectrum.

A slider-block model consists of a rectangular lattice of blocks that can slide in the twodimensional plane (see fig. A-1). Each block is connected by springs to its four nearest neighbors. In addition, there is a driving force applied to all the blocks. Because of the springs, whenever one block slides, the total force on each neighboring block increases, while the total force on the sliding block decreases. In other words, tectonic stress is transferred from the sliding block to its four neighboring blocks. (See Smalley and others, 1985; Huang and others, 1998.)

An examination of figure A-1 makes plain the similarity between the slider-block model and the Ising model. Both models consist of objects arranged in a lattice, where interactions occur only between nearest neighbors.

Our objective is to use the Boltzmann probability distribution to choose a random initial stress distribution on the fault surface. Because the Boltzmann probability of a state depends on the energy of the state, it is essential that each state be assigned a correct energy value. We now show that the slider-block model does not assign correct energy values and, therefore, that the slider-block model is not acceptable for this application.

Let $x_{0}$ and $x_{1}$ be coordinates on the plane. Suppose we impose a sinusoidal displacement on the blocks:

$$
u\left(x_{0}, x_{1}\right)=F \cos \left(k_{0} x_{0}+k_{1} x_{1}\right)
$$

where $u\left(x_{0}, x_{1}\right)$ is the displacement of the block located at coordinates $\left(x_{0}, x_{1}\right), F$ is an amplitude, and $k_{0}$ and $k_{1}$ are wave numbers in the two coordinate directions. Then the total energy stored in the springs scales as $k^{2}$ :

$$
E_{\text {spring }} \propto F^{2} k^{2} \quad \text { where } \quad k^{2}=k_{0}^{2}+k_{1}^{2} \text {. }
$$

But if you impose a sinusoidal pattern of slip on a fault embedded in a three-dimensional elastic medium, then the total energy stored in the elastic medium scales as $k$ :

$$
E_{\text {elastic }} \propto F^{2} k
$$

The difference can be understood as follows. In the case of the slider-block model, the springs are stretched or compressed by an amount proportional to $F k$, hence the energy in the springs is proportional to $(F k)^{2}$. In the case of a fault in a three-dimensional elastic medium, the medium immediately adjacent to the fault is distorted by an amount proportional to $F k$, hence the elastic energy density is proportional to $(F k)^{2}$. But the distortion penetrates into the threedimensional medium, perpendicular to the fault, by a distance proportional to the wavelength, which is $2 \pi / k$. So the amount of material subject to the distortion is proportional to $1 / k$, hence the total elastic energy is proportional to $(F k)^{2} / k$. 
Because the variation of energy with wavenumber is not correct, we cannot employ a slider-block model. 


\section{Appendix B. Properties of the Basis Displacement Fields}

In this appendix we establish the mathematical properties of the basis displacement fields, which were defined in equations 8-10.

We use a three-dimensional coordinate system $\left(x_{0}, x_{1}, x_{2}\right)$ where the $x_{0}$ direction is vertical, the $x_{1}$ direction is along-strike, and the $x_{2}$ direction is perpendicular to the fault. The fault is the surface $x_{2}=0$. We impose periodic boundary conditions in the $x_{0}$ and $x_{1}$ directions (but not in the $x_{2}$ direction), with periods $L_{0}$ and $L_{1}$ respectively.

A displacement is represented by a vector $\left(u_{0}, u_{1}, u_{2}\right)$. Our basis displacement fields are defined as:

$$
\begin{gathered}
u_{0}\left(x_{0}, x_{1}, x_{2}\right)=R\left(k_{0}, k_{1}\right) e^{-k\left|x_{2}\right|} \cos \left(k_{0} x_{0}+k_{1} x_{1}+\Phi\left(k_{0}, k_{1}\right)\right) \operatorname{sgn}\left(\mathrm{x}_{2}\right) \\
u_{1}\left(x_{0}, x_{1}, x_{2}\right)=F\left(k_{0}, k_{1}\right) e^{-k\left|x_{2}\right|} \cos \left(k_{0} x_{0}+k_{1} x_{1}+\Phi\left(k_{0}, k_{1}\right)\right) \operatorname{sgn}\left(x_{2}\right) \\
u_{2}\left(x_{0}, x_{1}, x_{2}\right)=-\frac{1}{k}\left(k_{0} R\left(k_{0}, k_{1}\right)+k_{1} F\left(k_{0}, k_{1}\right)\right) e^{-k\left|x_{2}\right|} \sin \left(k_{0} x_{0}+k_{1} x_{1}+\Phi\left(k_{0}, k_{1}\right)\right)
\end{gathered}
$$

Here $F\left(k_{0}, k_{1}\right)$ and $R\left(k_{0}, k_{1}\right)$ are arbitrary amplitudes, $\Phi\left(k_{0}, k_{1}\right)$ is an arbitrary phase, $k_{0}$ and $k_{1}$ are wavenumbers in the $x_{0}$ and $x_{1}$ directions, and $k$ is the scalar wavenumber defined as:

$$
k=\sqrt{k_{0}^{2}+k_{1}^{2}}
$$

The wavenumbers are assumed to be compatible with the periodic boundary conditions. In the main report (equations $8-10$ ), we defined $R\left(k_{0}, k_{1}\right)$ as follows:

$$
R\left(k_{0}, k_{1}\right)=-F\left(k_{0}, k_{1}\right) \frac{k_{0} k_{1}}{k^{2}+k_{0}^{2}}
$$

We will see below that this choice of $R$ causes the shear stress to be oriented along-strike. For most of this appendix, we allow $R$ to be arbitrary.

For the sake of brevity, we introduce the following abbreviations:

$$
\begin{gathered}
F \equiv F\left(k_{0}, k_{1}\right) \\
R \equiv R\left(k_{0}, k_{1}\right) \\
\psi \equiv k_{0} x_{0}+k_{1} x_{1}+\Phi\left(k_{0}, k_{1}\right) \\
\alpha \equiv k\left|x_{2}\right| \\
u_{0} \equiv u_{0}\left(k_{0}, k_{1}\right) \equiv u_{0}\left(x_{0}, x_{1}, x_{2}\right) \\
u_{1} \equiv u_{1}\left(k_{0}, k_{1}\right) \equiv u_{1}\left(x_{0}, x_{1}, x_{2}\right)
\end{gathered}
$$




$$
u_{2} \equiv u_{2}\left(k_{0}, k_{1}\right) \equiv u_{2}\left(x_{0}, x_{1}, x_{2}\right) .
$$

With these abbreviations, the basis displacement fields can be written as:

$$
\begin{gathered}
u_{0}=R e^{-\alpha} \cos (\psi) \operatorname{sgn}\left(\mathrm{x}_{2}\right) \\
u_{1}=F e^{-\alpha} \cos (\psi) \operatorname{sgn}\left(x_{2}\right) \\
u_{2}=-\frac{1}{k}\left(k_{0} R+k_{1} F\right) e^{-\alpha} \sin (\psi) .
\end{gathered}
$$

\section{Fault Slip and Exponential Decay}

The basis displacement fields are continuous everywhere except at the fault plane $x_{2}=0$, where there is a discontinuity due to the factor $\operatorname{sgn}\left(x_{2}\right)$. The fault slip vector $\left(s_{0}, s_{1}, s_{2}\right)$ is defined as:

$$
\begin{gathered}
s_{0} \equiv \lim _{x_{2} \rightarrow 0+} u_{0}-\lim _{x_{2} \rightarrow 0-} u_{0}=2 R \cos \psi \\
s_{1} \equiv \lim _{x_{2} \rightarrow 0+} u_{1}-\lim _{x_{2} \rightarrow 0-} u_{1}=2 F \cos \psi \\
s_{2} \equiv \lim _{x_{2} \rightarrow 0+} u_{2}-\lim _{x_{2} \rightarrow 0-} u_{2}=0 .
\end{gathered}
$$

We see that the basis displacement field describes a sinusoidal pattern of fault slip, with wavenumbers $k_{0}$ and $k_{1}$. The rake angle is determined by the relative size of the amplitudes $R$ and $F$.

The basis displacement fields depend on $x_{2}$ only through the factor $e^{-\alpha}=e^{-k\left|x_{2}\right|}$. So we see that the fields decay exponentially with increasing distance, $\left|x_{2}\right|$, from the fault surface, over a characteristic distance, $1 / k$.

\section{Derivatives of the Basis Displacement Fields}

We need to compute the derivatives of the basis displacement fields. First, note that the first derivatives of $\psi$ and $\alpha$ are (where we use a subscript comma to denote derivative):

$$
\begin{gathered}
\psi_{, 0}=k_{0} \\
\psi_{, 1}=k_{1} \\
\psi_{, 2}=0 \\
\alpha_{, 0}=\alpha_{, 1}=0 \\
\alpha_{, 2}=k \operatorname{sgn}\left(x_{2}\right) .
\end{gathered}
$$


Now we can write the first derivatives of the basis displacement fields:

$$
\begin{gathered}
u_{0,0}=-k_{0} R e^{-\alpha} \sin (\psi) \operatorname{sgn}\left(\mathrm{x}_{2}\right) \\
u_{0,1}=-k_{1} R e^{-\alpha} \sin (\psi) \operatorname{sgn}\left(\mathrm{x}_{2}\right) \\
u_{0,2}=-k R e^{-\alpha} \cos (\psi) \\
u_{1,0}=-k_{0} F e^{-\alpha} \sin (\psi) \operatorname{sgn}\left(\mathrm{x}_{2}\right) \\
u_{1,1}=-k_{1} F e^{-\alpha} \sin (\psi) \operatorname{sgn}\left(\mathrm{x}_{2}\right) \\
u_{1,2}=-k F e^{-\alpha} \cos (\psi) \\
u_{2,0}=-\frac{k_{0}}{k}\left(k_{0} R+k_{1} F\right) e^{-\alpha} \cos (\psi) \\
u_{2,1}=-\frac{k_{1}}{k}\left(k_{0} R+k_{1} F\right) e^{-\alpha} \cos (\psi) \\
u_{2,2}=\left(k_{0} R+k_{1} F\right) e^{-\alpha} \sin (\psi) \operatorname{sgn}\left(\mathrm{x}_{2}\right) .
\end{gathered}
$$

From the first derivatives, we see that

$$
u_{i, i}=0
$$

which means that the basis displacement fields are volume-preserving. It follows immediately that

$$
u_{i, i j}=u_{i, j i}=0 \quad \text { for all } j
$$

We can now write the second derivatives of the basis displacement fields:

$$
\begin{aligned}
& u_{0,00}=-k_{0}^{2} R e^{-\alpha} \cos (\psi) \operatorname{sgn}\left(\mathrm{x}_{2}\right) \\
& u_{0,11}=-k_{1}^{2} R e^{-\alpha} \cos (\psi) \operatorname{sgn}\left(\mathrm{x}_{2}\right) \\
& u_{0,22}=k^{2} R e^{-\alpha} \cos (\psi) \operatorname{sgn}\left(\mathrm{x}_{2}\right) \\
& u_{1,00}=-k_{0}^{2} F e^{-\alpha} \cos (\psi) \operatorname{sgn}\left(\mathrm{x}_{2}\right) \\
& u_{1,11}=-k_{1}^{2} F e^{-\alpha} \cos (\psi) \operatorname{sgn}\left(\mathrm{x}_{2}\right) \\
& u_{1,22}=k^{2} F e^{-\alpha} \cos (\psi) \operatorname{sgn}\left(\mathrm{x}_{2}\right)
\end{aligned}
$$




$$
\begin{aligned}
& u_{2,00}=\frac{k_{0}^{2}}{k}\left(k_{0} R+k_{1} F\right) e^{-\alpha} \sin (\psi) \\
& u_{2,11}=\frac{k_{1}^{2}}{k}\left(k_{0} R+k_{1} F\right) e^{-\alpha} \sin (\psi) \\
& u_{2,22}=-k\left(k_{0} R+k_{1} F\right) e^{-\alpha} \sin (\psi) .
\end{aligned}
$$

From the second derivatives, we see that

$$
u_{i, j j}=0 \quad \text { for all } i
$$

which means that the basis displacement fields satisfy the Laplace equation.

\section{Elastostatic Equation} tensor is

We show that the basis displacement fields satisfy the elastostatic equation. The strain

$$
\varepsilon_{i j}=\frac{1}{2}\left(u_{j, i}+u_{i, j}\right)
$$

The stress tensor is

$$
\sigma_{i j}=\lambda \delta_{i j} \varepsilon_{l l}+2 \mu \varepsilon_{i j}=\lambda \delta_{i j} u_{l, l}+\mu u_{i, j}+\mu u_{j, i}
$$

where $\mu$ is the shear modulus and $\lambda$ is the volumetric Lame parameter. Differentiating, we get

$$
\sigma_{i j, j}=\lambda \delta_{i j} u_{l, l j}+\mu u_{i, j j}+\mu u_{j, i j}=\mu u_{i, j j}+(\mu+\lambda) u_{j, i j}
$$

We have already shown that each of the terms on the right-hand side is equal to zero. So

$$
\sigma_{i j, j}=0
$$

which shows that the basis displacement fields satisfy the elastostatic equation.

\section{Elastic Energy}

Here we calculate the elastic energy associated with the basis displacement field. The elastic energy density is:

$$
\begin{aligned}
\mathcal{E} \equiv \mathcal{E}\left(k_{0}, k_{1}\right) & \equiv \frac{1}{2} \sigma_{i j} \varepsilon_{i j} \\
& =\frac{1}{2} \lambda\left(\varepsilon_{l l}\right)^{2}+\mu \varepsilon_{i j} \varepsilon_{i j} \\
& =\frac{1}{2} \lambda\left(u_{l, l}\right)^{2}+\frac{1}{2} \mu u_{i, j} u_{i, j}+\frac{1}{2} \mu u_{i, j} u_{j, i}
\end{aligned}
$$


To obtain the elastic energy, we must integrate $\mathcal{E}$ over all space. For our basis displacement fields, the first term on the right-hand side vanishes because we have shown that $u_{l, l}=0$. To evaluate the second term on the right-hand side, we group expressions as follows:

$$
\begin{gathered}
u_{0,0}^{2}+u_{0,1}^{2}+u_{0,2}^{2}=k^{2} R^{2} e^{-2 \alpha} \\
u_{1,0}^{2}+u_{1,1}^{2}+u_{1,2}^{2}=k^{2} F^{2} e^{-2 \alpha} \\
u_{2,0}^{2}+u_{2,1}^{2}+u_{2,2}^{2}=\left(k_{0} R+k_{1} F\right)^{2} e^{-2 \alpha} .
\end{gathered}
$$

Summing the above three equations, we get

$$
u_{i, j} u_{i, j}=\left(\left(k_{0} R+k_{1} F\right)^{2}+k^{2} R^{2}+k^{2} F^{2}\right) e^{-2 \alpha} .
$$

To evaluate the third term in the expression for $\mathcal{E}$, we group expressions as follows:

$$
\begin{gathered}
u_{2,2}^{2}=\left(k_{0} R+k_{1} F\right)^{2} e^{-2 \alpha} \sin ^{2}(\psi) \\
u_{0,0}^{2}+u_{1,1}^{2}+2 u_{0,1} u_{1,0}=\left(k_{0} R+k_{1} F\right)^{2} e^{-2 \alpha} \sin ^{2}(\psi) \\
2 u_{0,2} u_{2,0}+2 u_{1,2} u_{2,1}=2\left(k_{0} R+k_{1} F\right)^{2} e^{-2 \alpha} \cos ^{2}(\psi) .
\end{gathered}
$$

Summing the above three equations, we get

$$
u_{i, j} u_{j, i}=2\left(k_{0} R+k_{1} F\right)^{2} e^{-2 \alpha} .
$$

Combining the above, the elastic energy density is:

$$
\mathcal{E}=\frac{1}{2} \mu\left(3\left(k_{0} R+k_{1} F\right)^{2}+k^{2} R^{2}+k^{2} F^{2}\right) e^{-2 \alpha} .
$$

To obtain the total elastic energy, we must integrate $\mathcal{E}$ over all space. First, note that $\mathcal{E}$ is independent of $x_{0}$ and $x_{1}$. Because we impose periodic boundary conditions in the $x_{0}$ and $x_{1}$ directions, we have

$$
\begin{aligned}
& \int d x_{0}=L_{0} \\
& \int d x_{1}=L_{1} .
\end{aligned}
$$

Also,

$$
\int_{-\infty}^{\infty} e^{-2 \alpha} d x_{2}=\int_{-\infty}^{\infty} e^{-2 k\left|x_{2}\right|} d x_{2}=\frac{1}{k}
$$


Therefore the total elastic energy is:

$$
\begin{aligned}
E=E\left(k_{0}, k_{1}\right) & =\int \varepsilon\left(k_{0}, k_{1}\right) d x_{0} d x_{1} d x_{2} \\
& =\frac{1}{2} \mu L_{0} L_{1} k\left(3\left(\frac{k_{0}}{k} R+\frac{k_{1}}{k} F\right)^{2}+R^{2}+F^{2}\right) .
\end{aligned}
$$

For the special case where shear stress is oriented along-strike, we substitute $R=$ $-F k_{0} k_{1} /\left(k^{2}+k_{0}^{2}\right)$ to get the formula given in the main report (equation 15):

$$
E\left(k_{0}, k_{1}\right)=\frac{1}{2} \mu L_{0} L_{1} k\left(\frac{2 k^{2}}{k^{2}+k_{0}^{2}}\right)^{2} F^{2} .
$$

The above equation is derived using the following algebraic identity:

$$
\begin{aligned}
& 3\left(-\frac{k_{0}}{k} \frac{k_{0} k_{1}}{k^{2}+k_{0}^{2}}+\frac{k_{1}}{k}\right)^{2}+\frac{k_{0}^{2} k_{1}^{2}}{\left(k^{2}+k_{0}^{2}\right)^{2}}+1 \\
& =3\left(-\frac{1}{k} \frac{k_{0}^{2} k_{1}}{k^{2}+k_{0}^{2}}+\frac{1}{k} \frac{k_{1} k^{2}+k_{1} k_{0}^{2}}{k^{2}+k_{0}^{2}}\right)^{2}+\frac{k_{0}^{2} k_{1}^{2}}{\left(k^{2}+k_{0}^{2}\right)^{2}}+\frac{\left(k^{2}+k_{0}^{2}\right)^{2}}{\left(k^{2}+k_{0}^{2}\right)^{2}} \\
& =\frac{3\left(k_{1} k\right)^{2}+k_{0}^{2} k_{1}^{2}+k^{4}+2 k^{2} k_{0}^{2}+k_{0}^{4}}{\left(k^{2}+k_{0}^{2}\right)^{2}}=\frac{4 k^{4}}{\left(k^{2}+k_{0}^{2}\right)^{2}} .
\end{aligned}
$$

\section{Orthogonality}

We now show that the basis displacement fields are orthogonal. In other words, if we form the sum of the basis displacement fields, then the total elastic energy of the sum equals the sum of the elastic energies of the individual displacement fields.

Let $u_{i}^{\text {total }}$ be the sum of the basis displacement fields:

$$
u_{i}^{\text {total }}=\sum_{k_{0} k_{1}} u_{i}\left(k_{0}, k_{1}\right) .
$$

The sum runs over all wave vectors $\left(k_{0}, k_{1}\right)$ that are compatible with the periodic boundary conditions, but with the following two special considerations:

- Because wave vectors $\left(k_{0}, k_{1}\right)$ and $\left(-k_{0},-k_{1}\right)$ represent the same physical state, the sum includes only one member of each such pair of wave vectors.

- Because the zero wave vector $\left(k_{0}, k_{1}\right)=(0,0)$ represents a state with zero energy and zero stress, the sum does not include the zero wave vector.

Using the fact that $u_{l, l}^{\text {total }}=0$, we can write the total elastic energy density as: 


$$
\begin{aligned}
\mathcal{E}_{\mathrm{total}} & =\frac{1}{2} \mu u_{i, j}^{\mathrm{total}} u_{i, j}^{\mathrm{total}}+\frac{1}{2} \mu u_{i, j}^{\mathrm{total}} u_{j, i}^{\mathrm{total}} \\
& =\sum_{k_{0} k_{1}} \sum_{k_{0}^{\prime} k_{1}^{\prime}} \frac{1}{2} \mu\left(u_{i, j}\left(k_{0}, k_{1}\right) u_{i, j}\left(k_{0}^{\prime}, k_{1}^{\prime}\right)+u_{i, j}\left(k_{0}, k_{1}\right) u_{j, i}\left(k_{0}^{\prime}, k_{1}^{\prime}\right)\right) .
\end{aligned}
$$

The above expression is a pair of sums over $k_{0}, k_{1}, k_{0}^{\prime}, k_{1}^{\prime}, i$, and $j$. Each term in each sum has one of the following four functional forms:

$$
\begin{aligned}
& f\left(x_{2}, k_{0}, k_{1}, k_{0}^{\prime}, k_{1}^{\prime}\right) \sin \left(k_{0} x_{0}+k_{1} x_{1}+\Phi\left(k_{0}, k_{1}\right)\right) \sin \left(k_{0}^{\prime} x_{0}+k_{1}^{\prime} x_{1}+\Phi\left(k_{0}^{\prime}, k_{1}^{\prime}\right)\right) \\
& f\left(x_{2}, k_{0}, k_{1}, k_{0}^{\prime}, k_{1}^{\prime}\right) \sin \left(k_{0} x_{0}+k_{1} x_{1}+\Phi\left(k_{0}, k_{1}\right)\right) \cos \left(k_{0}^{\prime} x_{0}+k_{1}^{\prime} x_{1}+\Phi\left(k_{0}^{\prime}, k_{1}^{\prime}\right)\right) \\
& f\left(x_{2}, k_{0}, k_{1}, k_{0}^{\prime}, k_{1}^{\prime}\right) \cos \left(k_{0} x_{0}+k_{1} x_{1}+\Phi\left(k_{0}, k_{1}\right)\right) \sin \left(k_{0}^{\prime} x_{0}+k_{1}^{\prime} x_{1}+\Phi\left(k_{0}^{\prime}, k_{1}^{\prime}\right)\right) \\
& f\left(x_{2}, k_{0}, k_{1}, k_{0}^{\prime}, k_{1}^{\prime}\right) \cos \left(k_{0} x_{0}+k_{1} x_{1}+\Phi\left(k_{0}, k_{1}\right)\right) \cos \left(k_{0}^{\prime} x_{0}+k_{1}^{\prime} x_{1}+\Phi\left(k_{0}^{\prime}, k_{1}^{\prime}\right)\right) .
\end{aligned}
$$

If $\left(k_{0}, k_{1}\right) \neq\left(k_{0}^{\prime}, k_{1}^{\prime}\right)$ then each of the four expressions above integrates to zero, when integrated with respect to $x_{0}$ and $x_{1}$. Therefore, when we integrate $\varepsilon_{\text {total }}$ over all space, we only need to include the terms in the sum that have $\left(k_{0}, k_{1}\right)=\left(k_{0}^{\prime}, k_{1}^{\prime}\right)$. So the total elastic energy is:

$$
\begin{aligned}
E_{\text {total }} & =\int \varepsilon_{\text {total }}\left(k_{0}, k_{1}\right) d x_{0} d x_{1} d x_{2} \\
& =\sum_{k_{0} k_{1}} \int \frac{1}{2} \mu\left(u_{i, j}\left(k_{0}, k_{1}\right) u_{i, j}\left(k_{0}, k_{1}\right)+u_{i, j}\left(k_{0}, k_{1}\right) u_{j, i}\left(k_{0}, k_{1}\right)\right) d x_{0} d x_{1} d x_{2} \\
& =\sum_{k_{0} k_{1}} \int \varepsilon\left(k_{0}, k_{1}\right) d x_{0} d x_{1} d x_{2}=\sum_{k_{0} k_{1}} E\left(k_{0}, k_{1}\right) .
\end{aligned}
$$

This proves orthogonality.

\section{Normal Stress} plane:

We define the normal stress $\sigma$ to be the compressive stress perpendicular to the fault

$$
\sigma \equiv-\sigma_{22}=-\lambda u_{l, l}-\mu u_{2,2}=-\mu\left(k_{0} R+k_{1} F\right) e^{-\alpha} \sin (\psi) \operatorname{sgn}\left(\mathrm{x}_{2}\right)
$$

where we have used the fact that $u_{l, l}=0$.

We see that the normal stress $\sigma$ is not continuous at the fault surface $x_{2}=0$. This shows that the basis displacement fields are only an approximate solution to the elastostatic fault slip problem.

Note: In the special case, $k_{0} R+k_{1} F=0$, the normal stress is continuous at the fault surface, and so the basis displacement fields are an exact solution to the elastostatic fault slip 
problem. The condition $k_{0} R+k_{1} F=0$ holds if the wave vector is perpendicular to the slip vector.

Note: We make this approximation because we are not aware of any analytic formulas similar to equations 82-84 that give the displacement field throughout space, for the exact solution of the elastostatic fault slip problem with a sinusoidal slip pattern.

Note: One can potentially perform a similar calculation to the one given here, without making this approximation, despite the lack of analytic solutions. Given a sinusoidal pattern of fault slip, the resulting shear stress on the fault plane could be calculated using integral formulas similar to those in Andrews (1974) (although it should be noted that the integrals in Andrews (1974) are divergent for sinusoidal fault slip). Then, total elastic energy could be computed by integrating the dot product of slip and shear stress over the fault plane.

\section{Shear Stress}

Let $\tau_{0}$ and $\tau_{1}$ be the components of shear stress in the $x_{0}$ direction (along-dip) and $x_{1}$ direction (along-strike), respectively. Their values are:

$$
\begin{aligned}
\tau_{0} \equiv \sigma_{02} & =\mu\left(u_{0,2}+u_{2,0}\right) \\
& =-\mu k\left(\frac{k^{2}+k_{0}^{2}}{k^{2}} R+\frac{k_{0} k_{1}}{k^{2}} F\right) e^{-\alpha} \cos (\psi) \\
\tau_{1} \equiv \sigma_{12} & =\mu\left(u_{1,2}+u_{2,1}\right) \\
& =-\mu k\left(\frac{k_{0} k_{1}}{k^{2}} R+\frac{k^{2}+k_{1}^{2}}{k^{2}} F\right) e^{-\alpha} \cos (\psi) .
\end{aligned}
$$

We see that the shear stress components $\tau_{0}$ and $\tau_{1}$ are continuous at the fault surface $x_{2}=0$, as stated in the main report.

From here on we are only concerned with the values of shear stress at the fault surface, so we drop the exponential term $e^{-\alpha}$, which equals 1 when $x_{2}=0$.

If $\tau_{0}=0$, then the shear stress is oriented along-strike. From the above formulas, we see this happens when

$$
R=-F \frac{k_{0} k_{1}}{k^{2}+k_{0}^{2}}
$$

Notice that the rake angle (the angle between the horizontal and the shear stress) can be set to any desired angle by adjusting the values of $R$ and $F$. To prove this fact, note that given any numbers $A_{0}$ and $A_{1}$, it is always possible to find values of $R$ and $F$ that satisfy the simultaneous linear equations 


$$
\begin{aligned}
& \frac{k^{2}+k_{0}^{2}}{k^{2}} R+\frac{k_{0} k_{1}}{k^{2}} F=A_{0} \\
& \frac{k_{0} k_{1}}{k^{2}} R+\frac{k^{2}+k_{1}^{2}}{k^{2}} F=A_{1}
\end{aligned}
$$

because of the non-vanishing of the determinant

$$
\left|\begin{array}{cc}
\frac{k^{2}+k_{0}^{2}}{k^{2}} & \frac{k_{0} k_{1}}{k^{2}} \\
\frac{k_{0} k_{1}}{k^{2}} & \frac{k^{2}+k_{1}^{2}}{k^{2}}
\end{array}\right|=2 k^{2} .
$$

Let $\tau$ be the two-dimensional shear stress. Then

$$
\begin{aligned}
\tau & = \pm\left(\tau_{0}^{2}+\tau_{1}^{2}\right)^{1 / 2} \\
& = \pm \mu k\left(3\left(\frac{k_{0}}{k} R+\frac{k_{1}}{k} F\right)^{2}+R^{2}+F^{2}\right)^{1 / 2} \cos (\psi)
\end{aligned}
$$

where the sign of $\tau$ can be chosen arbitrarily. To derive the above formula, one needs to use the algebraic identity

$$
\begin{aligned}
\left(\left(k^{2}+k_{0}^{2}\right) R+k_{0} k_{1} F\right)^{2} & +\left(k_{0} k_{1} R+\left(k^{2}+k_{1}^{2}\right) F\right)^{2} \\
& =k^{2}\left(3\left(k_{0} R+k_{1} F\right)^{2}+k^{2} R^{2}+k^{2} F^{2}\right)
\end{aligned}
$$

which can be verified by making the substitution $k^{2} \rightarrow k_{0}^{2}+k_{1}^{2}$ and then expanding both sides of the identity.

We previously showed that the elastic energy is

$$
E=\frac{1}{2} \mu L_{0} L_{1} k\left(3\left(\frac{k_{0}}{k} R+\frac{k_{1}}{k} F\right)^{2}+R^{2}+F^{2}\right) .
$$

Therefore the shear stress is

$$
\tau= \pm \sqrt{\frac{2 E \mu k}{L_{0} L_{1}}} \cos (\psi)
$$

Notice that the relation between the elastic energy $E$ and the shear stress $\tau$ is independent of the rake angle. Because the Boltzmann probability distribution depends on energy, this implies that the Boltzmann probability distribution cannot provide any information about the 
rake angle. So, we must impose a rake angle, such as by requiring the shear stress to be oriented along-strike.

For the special case where shear stress is oriented along-strike, we fix the sign of $\tau$ by requiring that $\tau=\tau_{1}$. Substituting $R=-F k_{0} k_{1} /\left(k^{2}+k_{0}^{2}\right)$ into the formula for $\tau_{1}$, we get

$$
\begin{aligned}
\tau_{1} & =-\mu k\left(-\frac{k_{0}^{2} k_{1}^{2}}{k^{2}\left(k^{2}+k_{0}^{2}\right)}+\frac{k^{2}+k_{1}^{2}}{k^{2}}\right) F \cos (\psi) \\
& =-\mu k \frac{2 k^{2}}{k^{2}+k_{0}^{2}} F \cos (\psi) .
\end{aligned}
$$

We see that for $\tau$ to have the same sign as $\tau_{1}$, we must have

$$
\tau=-\sqrt{\frac{2 E \mu k}{L_{0} L_{1}}} \cos (\psi) \operatorname{sgn}(F)
$$

which is equation 20 in the main report.

The mean-square value of the two-dimensional shear stress $\tau$, which we denote $\tau_{\text {rms }}^{2}$, is:

$$
\tau_{\mathrm{rms}}^{2} \equiv \frac{1}{L_{0} L_{1}} \int\left(\tau\left(x_{0}, x_{1}\right)\right)^{2} d x_{0} d x_{1}=\frac{E \mu k}{L_{0} L_{1}}
$$

which is equation 21 in the main report.

\section{Total Shear Stress and Completeness} strike.

In this part, we confine our attention to the case where shear stress is oriented along-

As before, let $u_{i}^{\text {total }}$ be the sum of the basis displacement fields:

$$
u_{i}^{\text {total }}=\sum_{k_{0} k_{1}} u_{i}\left(k_{0}, k_{1}\right)
$$

The sum runs over all wave vectors $\left(k_{0}, k_{1}\right)$ that are compatible with the periodic boundary conditions, with the special considerations described earlier. The shear stress produced by $u_{i}^{\text {total }}$ is:

$$
\begin{aligned}
\tau_{\text {total }}\left(x_{0}, x_{1}\right) & =\sum_{k_{0} k_{1}} \tau\left(k_{0}, k_{1}\right) \\
& =-\sum_{k_{0} k_{1}} \sqrt{\frac{2 E\left(k_{0}, k_{1}\right) \mu k}{L_{0} L_{1}}} \cos \left(k_{0} x_{0}+k_{1} x_{1}+\Phi\left(k_{0}, k_{1}\right)\right) \operatorname{sgn}(F) .
\end{aligned}
$$


This equation has the form of a two-dimensional Fourier transform. It implies that the basis displacement fields are complete in the following sense: Given any pattern of shear stress on the fault surface, oriented along-strike and with a finite mean-square, it is possible to find some linear combination of the basis displacement fields that produces that pattern of shear stress. The linear combination can be found by performing a Fourier transform on the desired pattern of shear stress.

The equation also implies that the basis displacement fields are independent in the following sense: Any two different linear combinations of the basis displacement fields produce different patterns of shear stress on the fault.

We can apply Parseval's theorem to compute the mean-square value of $\tau_{\text {total }}$, which we denote by $\tau_{\text {total }}^{2}$ :

$$
\tau_{\text {total }}^{2} \equiv \frac{1}{L_{0} L_{1}} \int\left(\tau_{\text {total }}\left(x_{0}, x_{1}\right)\right)^{2} d x_{0} d x_{1}=\sum_{k_{0} k_{1}} \frac{E\left(k_{0}, k_{1}\right) \mu k}{L_{0} L_{1}}=\sum_{k_{0} k_{1}} \tau_{\text {rms }}^{2}\left(k_{0}, k_{1}\right)
$$

In other words, the mean-square value of the total shear stress equals the sum of the mean-square values of the individual basis displacement fields. This formula (in integral form) appears as equation 22 in the main report.

Finally, we note that given a Fourier expansion, the power spectral density is defined to be the square of the magnitude of the Fourier coefficients. We see that $\tau_{\mathrm{rms}}^{2}\left(k_{0}, k_{1}\right)$ is the power spectral density of $\tau_{\text {total }}$. In other words, the mean-square shear stresses of the individual basis displacement fields are equal to the power spectral density of the total shear stress. 


\section{Appendix C. Derivation of the Earthquake Rate Formula} relations.

In this appendix we show how to derive the earthquake rate formula from the scaling

We start by stating three earthquake scaling relations from Hanks (1977; 1979). We give them in a slightly more general form than in the main report.

Scaling relation 1 is the Gutenberg-Richter relation (Gutenberg and Richter, 1954). Let $M$ be an earthquake magnitude, and let $N$ be the rate of occurrence of earthquakes with magnitude $\geq M$. Then

$$
\log _{10} N=a-b M
$$

where $a$ and $b$ are some constants.

Scaling relation 2 is the relation between magnitude and seismic moment (Hanks and Kanamori, 1979). Let $M_{0}$ be seismic moment, which is defined as

$$
M_{0}=\kappa \delta r^{2}
$$

where $\delta$ is fault slip, $r$ is the radius of the fault rupture area, and $\kappa$ is some constant. It is conventional to take $\kappa=\pi \mu$ where $\mu$ is the shear modulus, but this particular choice of $\kappa$ is not necessary for the present discussion. Then

$$
\log _{10} M_{0}=c M+d
$$

where $c$ and $d$ are some constants.

Scaling relation 3 is the statement that earthquakes are self-similar or scale-invariant. It is

$$
\delta=\gamma r
$$

where $\gamma$ is some constant.

Substituting equation 172 into equation 170, we have

$$
M_{0}=\kappa \gamma r^{3} \text {. }
$$

Substituting this into equation 171 and solving for $M$, we get

$$
M=\frac{1}{c} \log _{10}\left(\kappa \gamma r^{3}\right)-\frac{d}{c}
$$

Substituting this into equation 169 , we get

$$
\log _{10} N=\left(a+\frac{b d}{c}\right)-\frac{b}{c} \log _{10}\left(\kappa \gamma r^{3}\right)
$$

Differentiate the above formula with respect to $r$ to obtain 


$$
\frac{1}{N} \frac{d N}{d r}=-\frac{3 b}{c r}
$$

The above differential equation for $N$ as a function of $r$ has solution

$$
\begin{gathered}
N=\eta r^{-3 b / c} \\
\frac{d N}{d r}=-\frac{3 b \eta}{c} r^{-3 b / c-1}
\end{gathered}
$$

where $\eta$ is some constant. Note that $\eta>0$ because the earthquake rate, $N$, decreases as the radius $r$ increases.

Substituting in the empirical values $b=1$ and $c=1.5$, we obtain equation 42 in the main report:

$$
-\frac{d N}{d r} \propto r^{-3}
$$




\section{Appendix D. Derivation of the Power Spectral Density}

In the main report (equation 47) we demonstrated a scaling relation for stress perturbations on the fault surface. We showed that

$$
A(r \leq R) \propto R
$$

where $A(r \leq R)$ denotes the total area of the fault surface occupied by positive stress perturbations of radius $\leq R$.

In this appendix we derive the power spectral density of stress, which is consistent with the above scaling relation. Our end result will be a formula that gives the asymptotic behavior of the power spectral density, in the limit of large wavenumber.

\section{Notations and Definitions}

First we recall some notations from the main report. The fault plane has coordinates $x_{0}$ and $x_{1}$, where $x_{0}$ runs vertically and $x_{1}$ runs along-strike. We impose periodic boundary conditions, with periods $L_{0}$ and $L_{1}$, so that the fault area is $L_{0} L_{1}$. We use $k_{0}$ and $k_{1}$ to denote wavenumbers in the two coordinate directions, and the scalar wavenumber is $k=\sqrt{k_{0}^{2}+k_{1}^{2}}$.

We denote the shear stress pattern on the fault as $\tau_{\text {total }}\left(x_{0}, x_{1}\right)$. We think of it as being the sum of plane waves (essentially the same thing as a Fourier transform):

$$
\tau_{\text {total }}\left(x_{0}, x_{1}\right)=\frac{L_{0} L_{1}}{4 \pi^{2}} \int \tau\left(k_{0}, k_{1}\right) d k_{0} d k_{1}
$$

The constant $L_{0} L_{1} / 4 \pi^{2}$ is called the density of states and is explained in the main report (equation 18); its value is not important for the present discussion. The individual plane waves take the form

$$
\tau\left(k_{0}, k_{1}\right)=Z\left(k_{0}, k_{1}\right) \cos \left(k_{0} x_{0}+k_{1} x_{1}+\Phi\left(k_{0}, k_{1}\right)\right)
$$

where $Z\left(k_{0}, k_{1}\right)$ and $\Phi\left(k_{0}, k_{1}\right)$ are random amplitudes and phases, respectively.

Let $\tau_{\text {rms }}^{2}\left(k_{0}, k_{1}\right)$ be the mean-square value of the individual plane wave. In the above notation, it is $\left(Z\left(k_{0}, k_{1}\right)\right)^{2} / 2$. Then $\tau_{\text {rms }}^{2}\left(k_{0}, k_{1}\right)$ is the (two-dimensional) power spectral density of the stress pattern.

The shear stress distribution on the fault is generated by some random process. Whenever we use the word "expected" or enclose an expression in angle brackets, we mean the average value obtained over many such randomly generated stress distributions. For example, $\tau_{\text {rms }}^{2}\left(k_{0}, k_{1}\right)$ denotes the power spectral density for some particular stress distribution, while $\left\langle\tau_{\text {rms }}^{2}\left(k_{0}, k_{1}\right)\right\rangle$ denotes its expected or average value over many stress distributions.

We assume that the expected power spectral density $\left\langle\tau_{\text {rms }}^{2}\left(k_{0}, k_{1}\right)\right\rangle$ is isotropic, that is, it depends on the scalar wavenumber $k$ but not on the direction of the wave vector. Note that the power spectral density $\tau_{\text {rms }}^{2}\left(k_{0}, k_{1}\right)$ for a single stress distribution is not isotropic. Only its expected value $\left\langle\tau_{\text {rms }}^{2}\left(k_{0}, k_{1}\right)\right\rangle$ is isotropic. This, in part, is why we must work with expected values and not individual stress distributions.

The expected mean-square value of the shear stress is defined to be 


$$
\left\langle\tau_{\text {total }}^{2}\right\rangle \equiv\left\langle\frac{1}{L_{0} L_{1}} \int\left(\tau_{\text {total }}\left(x_{0}, x_{1}\right)\right)^{2} d x_{0} d x_{1}\right\rangle
$$

Using Parseval's theorem, this can be written as

$$
\left\langle\tau_{\text {total }}^{2}\right\rangle=\left\langle\frac{L_{0} L_{1}}{4 \pi^{2}} \int \tau_{\text {rms }}^{2}\left(k_{0}, k_{1}\right) d k_{0} d k_{1}\right\rangle=\frac{L_{0} L_{1}}{4 \pi} \int_{0}^{\infty}\left\langle\tau_{\text {rms }}^{2}\left(k_{0}, k_{1}\right)\right\rangle k d k
$$

where the final integral depends on the fact that $\left\langle\tau_{\mathrm{rms}}^{2}\left(k_{0}, k_{1}\right)\right\rangle$ is isotropic. The constants appearing in front of the integrals are explained in the main report (equations 18 and 19); their values are not important for the present discussion.

\section{Filtered Stress Fields}

Next we introduce the concept of a filtered stress field, which contains only the highfrequency components of the stress distribution. This is useful because the high-frequency components of the stress distribution are responsible for the small-scale stress perturbations, which enter into the scaling relation. Also, we are trying to find the asymptotic behavior of the expected power spectral density, in the limit of large wavenumber.

Let $\tau_{\text {total }}\left(x_{0}, x_{1} ; k \geq K\right)$ denote the portion of the stress field that is due to wavenumbers $\geq K$. In other words, $\tau_{\text {total }}\left(x_{0}, x_{1} ; k \geq K\right)$ is the result of applying a high-pass spatial filter to the stress distribution $\tau_{\text {total }}\left(x_{0}, x_{1}\right)$, chosen so that the filter only passes wavenumbers $\geq K$. If $\tau_{\text {total }}\left(x_{0}, x_{1}\right)$ is constructed by forming the sum of plane waves with various wave vectors, then $\tau_{\text {total }}\left(x_{0}, x_{1} ; k \geq K\right)$ is constructed by forming the sum of the same plane waves, except that only those plane waves with wavenumbers $\geq K$ are included in the sum. Using the notations introduced above,

$$
\tau_{\text {total }}\left(x_{0}, x_{1} ; k \geq K\right) \equiv \frac{L_{0} L_{1}}{4 \pi^{2}} \int_{k_{0}^{2}+k_{1}^{2} \geq K^{2}} \tau\left(k_{0}, k_{1}\right) d k_{0} d k_{1}
$$

The expected mean-square value of the filtered shear stress $\tau_{\text {total }}\left(x_{0}, x_{1} ; k \geq K\right)$ is:

$$
\left\langle\tau_{\text {total }}^{2}(k \geq K)\right\rangle=\frac{L_{0} L_{1}}{4 \pi} \int_{K}^{\infty}\left\langle\tau_{\text {rms }}^{2}\left(k_{0}, k_{1}\right)\right\rangle k d k
$$

Notice that the limits of integration run from $K$ to $\infty$ because the filtered stress only includes wavenumbers $\geq K$.

\section{Expected One-Point Statistics of Filtered Stress}

We need to know more about the filtered stress fields than the expected mean-square value. To do this, we study their expected one-point statistics, which is the expected statistical distribution of stress values at individual points on the fault surface. 
For each $K>0$, define $X_{K}$ to be a real-valued random variable which has the expected one-point statistics of the filtered stress field $\tau_{\text {total }}\left(x_{0}, x_{1} ; k \geq K\right)$. What this definition means is that the cumulative density function of $X_{K}$ is defined to be:

$$
P\left(X_{K} \geq \xi\right) \equiv\left\langle\frac{1}{L_{0} L_{1}} v\left(\tau_{\text {total }}\left(x_{0}, x_{1} ; k \geq K\right) \geq \xi\right)\right\rangle
$$

where $P\left(X_{K} \geq \xi\right)$ is the probability that a randomly selected value of $X_{K}$ is greater than or equal to $\xi$, and where $v\left(\tau_{\text {total }}\left(x_{0}, x_{1} ; k \geq K\right) \geq \xi\right)$ denotes the area of the portion of the fault surface where the filtered stress field $\tau_{\text {total }}\left(x_{0}, x_{1} ; k \geq K\right)$ is greater than or equal to $\xi$.

In other words, the probability that $X_{K}$ is greater than or equal to $\xi$ is equal to the expected fraction of the fault surface where the filtered stress $\tau_{\text {total }}\left(x_{0}, x_{1} ; k \geq K\right)$ is greater than or equal to $\xi$.

With this definition, the mean of $X_{K}$ is zero, and the variance of $X_{K}$ is the expected meansquare stress:

$$
\begin{gathered}
\operatorname{mean}\left(X_{K}\right)=0 \\
\operatorname{var}\left(X_{K}\right)=\left\langle\tau_{\text {total }}^{2}(k \geq K)\right\rangle=\frac{L_{0} L_{1}}{4 \pi} \int_{K}^{\infty}\left\langle\tau_{\text {rms }}^{2}\left(k_{0}, k_{1}\right)\right\rangle k d k .
\end{gathered}
$$

The proof of these formulas is lengthy. A subsection at the end of this appendix shows how to prove these formulas, and also addresses some technical issues related to the definition of $X_{K}$.

\section{Small-Scale Stress Perturbations}

Now we need to make the connection between $X_{K}$ and the total area of small-scale positive stress perturbations, which is what appears in our scaling relation.

Because $\tau_{\text {total }}\left(x_{0}, x_{1} ; k \geq K\right)$ contains all wavelengths $\leq 2 \pi / K$, we say that $\tau_{\text {total }}\left(x_{0}, x_{1} ; k \geq K\right)$ contains all stress perturbations of radius $\leq \gamma / K$ for some constant $\gamma$. (It would be reasonable to take $\gamma=1$, but, as we will see, the value of $\gamma$ has no effect on our final result.)

We say that a positive stress perturbation occurs when the stress $\tau_{\text {total }}\left(x_{0}, x_{1} ; k \geq K\right)$ equals or exceeds some constant level $C$. Then, the total area of all positive stress perturbations of radius $\leq \gamma / K$ is

$$
A(r \leq \gamma / K)=v\left(\tau_{\text {total }}\left(x_{0}, x_{1} ; k \geq K\right) \geq C\right) .
$$

Notice that the threshold stress level $C$ is independent of $K$. This is a key assumption. It comes from assumption of earthquake self-similarity, which states that the stress drop in an earthquake is independent of the size of the rupture area.

The expected total area of all positive stress perturbations of radius $\leq \gamma / K$ is

$$
\langle A(r \leq \gamma / K)\rangle=\left\langle v\left(\tau_{\text {total }}\left(x_{0}, x_{1} ; k \geq K\right) \geq C\right)\right\rangle=L_{0} L_{1} P\left(X_{K} \geq C\right) .
$$


As $K$ varies, we require the expected area to vary in accordance with our scaling relation

$$
\langle A(r \leq \gamma / K)\rangle \propto \gamma / K .
$$

Combining the above two equations, we obtain the scaling relation

$$
P\left(X_{K} \geq C\right) \propto 1 / K
$$

where the constants $L_{0}, L_{1}$, and $\gamma$ have been absorbed into the implied constant of proportionality. This is the scaling relation we use in the calculations to follow.

\section{Method of Calculation}

Here we explain the method we will use to start with the scaling relation above, and calculate the expected power spectral density.

For convenience, let us define $V$ by

$$
V \equiv V(K) \equiv \operatorname{var}\left(X_{K}\right) .
$$

Then, recalling that the expected power spectral density $\left\langle\tau_{\mathrm{rms}}^{2}\left(k_{0}, k_{1}\right)\right\rangle$ is isotropic, we have

$$
\begin{gathered}
V(K)=\frac{L_{0} L_{1}}{4 \pi} \int_{K}^{\infty}\left\langle\tau_{\mathrm{rms}}^{2}\left(k_{0}, k_{1}\right)\right\rangle k d k \\
\frac{d V}{d K}=-\frac{L_{0} L_{1}}{4 \pi}\left\langle\tau_{\mathrm{rms}}^{2}\left(K_{0}, K_{1}\right)\right\rangle K
\end{gathered}
$$

and so the expected power spectral density is:

$$
\left\langle\tau_{\mathrm{rms}}^{2}\left(K_{0}, K_{1}\right)\right\rangle \propto-\frac{1}{K} \frac{d V}{d K} .
$$

Our objective is to find $d V / d K$ asymptotically in the limit $K \rightarrow \infty$. Then, the above formula gives an asymptotic expression for the expected power spectral density $\left\langle\tau_{\mathrm{rms}}^{2}\left(K_{0}, K_{1}\right)\right\rangle$.

Let us rewrite our scaling relation with an explicit constant of proportionality $\alpha$ :

$$
P\left(X_{K} \geq C\right)=\alpha / K .
$$

Notice that $\alpha$ has dimensions of inverse length, because the wavenumber $K$ has dimensions of inverse length.

The remainder of the calculation proceeds as follows. First, we make an assumption about the probability density function of the random variables $X_{K}$. Then, we start from the scaling relation and derive a formula for $V$ or $d V / d K$. If it is not possible to derive an exact formula in closed form, then we derive a formula that is asymptotically true in the limit $K \rightarrow \infty$. 
Then, we immediately obtain an asymptotic formula for the expected power spectral density $\left\langle\tau_{\text {rms }}^{2}\left(K_{0}, K_{1}\right)\right\rangle$.

We will first assume that each $X_{K}$ is a Gaussian random variable. This assumption is the most physically reasonable, and it yields the formula given in the main report (equation 48). Then, to explore the sensitivity of our results to the assumed probability distribution of $X_{K}$, we repeat the calculation using three other probability distributions: the Laplace, exponential, and Pareto distributions. We will see that the Gaussian, Laplace, and exponential distributions, which have tails that fall off exponentially, all produce very similar results: the power spectral density falls off as $1 / K^{2}$ modified by a logarithmic term. For the Pareto distribution, which has a tail that falls off as a power law, the power spectral density falls off as a power law that is faster than $1 / K^{2}$, but slower than $1 / K^{3}$. The implication of these results, especially the three with exponential tails, is that the power spectral density is not very sensitive to the precise probability distribution of the random variable $X_{K}$.

Before beginning, a word of warning about the manipulation of asymptotic expressions is needed. We say that functions $f_{1}(x)$ and $f_{2}(x)$ are asymptotic in the limit $x \rightarrow \infty$, and we write $f_{1}(x) \sim f_{2}(x)$, if and only if, $f_{1}(x) / f_{2}(x) \rightarrow 1$ in the limit $x \rightarrow \infty$. Some operations that are valid on equalities are not valid on asymptotic relations. For example, if $f_{1}(x) \sim f_{2}(x)$, then it follows that $\log f_{1}(x) \sim \log f_{2}(x)$, but not that $\exp f_{1}(x) \sim \exp f_{2}(x)$. In particular, it is not generally valid to differentiate an asymptotic relation; from $f_{1}(x) \sim f_{2}(x)$, it does not follow that $f_{1}^{\prime}(x) \sim f_{2}^{\prime}(x)$.

\section{Calculation Assuming Gaussian Distribution}

Here we assume that each $X_{K}$ is a Gaussian random variable, with zero mean and variance $V=V(K)$.

We begin with some preliminaries. The complementary error function is defined as

$$
\operatorname{erfc}(x) \equiv \frac{2}{\sqrt{\pi}} \int_{x}^{\infty} e^{-t^{2}} d t .
$$

Its derivative is

$$
\frac{d}{d x} \operatorname{erfc}(x)=-\frac{2}{\sqrt{\pi}} e^{-x^{2}}
$$

The complementary error function, and its inverse, have asymptotic formulas (National Institute of Standards and Technology, 2011):

$$
\begin{gathered}
\operatorname{erfc}(x) \sim \frac{1}{\sqrt{\pi} x} e^{-x^{2}} \quad \text { as } x \rightarrow \infty \\
\operatorname{inverfc}(y) \sim\left(-\frac{1}{2} \ln \left(\pi y^{2} \ln (1 / y)\right)\right)^{1 / 2} \quad \text { as } y \rightarrow 0 .
\end{gathered}
$$

For our purposes, we use a simpler (albeit more slowly converging) asymptotic formula for the inverse complementary error function, 


$$
\operatorname{inverfc}(y) \sim\left(\ln \left(\frac{1}{\sqrt{\pi} y}\right)\right)^{1 / 2} \quad \text { as } y \rightarrow 0 .
$$

This is also a valid asymptotic formula because

$$
\ln \left(\pi y^{2} \ln (1 / y)\right)=\ln \left(\pi y^{2}\right)+\ln (\ln (1 / y)) \sim \ln \left(\pi y^{2}\right) .
$$

This completes the preliminaries. If $X_{K}$ is Gaussian with zero mean and variance $V$, then our scaling relation

$$
P\left(X_{K} \geq C\right)=\alpha / K
$$

Becomes

$$
\frac{\alpha}{K}=\frac{1}{2} \operatorname{erfc}\left(\frac{C}{\sqrt{2 V}}\right)
$$

Differentiate the above equation with respect to $K$, using the formula for the derivative of the complementary error function, to obtain

$$
-\frac{\alpha}{K^{2}}=\frac{1}{C^{2}}\left(\frac{C}{\sqrt{2 V}}\right)^{4}\left(\frac{\sqrt{2 V}}{\sqrt{\pi} C} e^{-C^{2} / 2 V}\right) \frac{d V}{d K}
$$

Noting that $V \rightarrow 0$ as $K \rightarrow \infty$, use the asymptotic formula for the complementary error function to get

$$
\frac{2 \alpha}{K}=\operatorname{erfc}\left(\frac{C}{\sqrt{2 V}}\right) \sim \frac{\sqrt{2 V}}{\sqrt{\pi} C} e^{-C^{2} / 2 V} \quad \text { as } K \rightarrow \infty .
$$

Using the asymptotic formula for the inverse complementary error function,

$$
\frac{C}{\sqrt{2 V}}=\operatorname{inverfc}\left(\frac{2 \alpha}{K}\right) \sim(\ln (\zeta K))^{1 / 2} \quad \text { as } K \rightarrow \infty
$$

where $\zeta \equiv 1 / 2 \sqrt{\pi} \alpha$ is a parameter with dimensions of length.

(From a strictly mathematical standpoint, one could eliminate $\zeta$ and write $\ln (K)$ in place of $\ln (\zeta K)$, and still have a correct asymptotic formula. We retain $\zeta$ because the wavenumber $K$ has dimensions of inverse length, while the logarithm function requires a dimensionless argument, so it would be physically incorrect to omit $\zeta$.)

Combining the three previous formulas,

$$
-\frac{\alpha}{K^{2}} \sim \frac{1}{C^{2}}(\ln (\zeta K))^{2}\left(\frac{2 \alpha}{K}\right) \frac{d V}{d K} \quad \text { as } K \rightarrow \infty
$$


which can be re-written as

$$
-\frac{1}{K} \frac{d V}{d K} \sim \frac{C^{2}}{2 K^{2}(\ln (\zeta K))^{2}} \quad \text { as } K \rightarrow \infty
$$

So the expected power spectral density is

$$
\left\langle\tau_{\mathrm{rms}}^{2}\left(K_{0}, K_{1}\right)\right\rangle \propto-\frac{1}{K} \frac{d V}{d K} \propto \frac{1}{K^{2}(\ln (\zeta K))^{2}} \quad \text { as } K \rightarrow \infty .
$$

This is equation 48 in the main report.

Remark: It is interesting to note that, if it were permitted to differentiate an asymptotic formula, the above result could be obtained much more quickly. Using the asymptotic formula for the inverse complementary error function,

$$
V=\frac{C^{2}}{2}\left(\operatorname{inverfc}\left(\frac{2 \alpha}{K}\right)\right)^{-2} \sim \frac{C^{2}}{2}(\ln (\zeta K))^{-1} \quad \text { as } K \rightarrow \infty .
$$

Differentiating the right-hand side of the above formula with respect to $K$, were it permissible to do so, would immediately yield the same asymptotic result for $d V / d K$.

\section{Calculation Assuming Laplace Distribution}

Here we assume that each $X_{K}$ is a Laplace random variable, with zero mean and variance $V=V(K)$. The Laplace distribution is a symmetric double-tailed distribution, with probability density proportional to $\exp (-\lambda|t|)$. By comparison, the Gaussian distribution is a symmetric double-tailed distribution with a probability density proportional to $\exp \left(-t^{2} / 2 \sigma^{2}\right)$. So, this calculation explores the effect of using a distribution that falls off more slowly than Gaussian.

The Laplace distribution is defined to have the cumulative density function

$$
P\left(X_{K} \geq C\right)=\frac{1}{2} e^{-C / \sqrt{V}} \quad \text { assuming } C \geq 0 \text {. }
$$

The restriction on $C$ is not a problem, because $C$ is the threshold stress for defining positive stress perturbations, so physically we will always have $C \geq 0$.

Our scaling relation $P\left(X_{K} \geq C\right)=\alpha / K$ becomes

$$
\frac{\alpha}{K}=\frac{1}{2} e^{-C / \sqrt{V}}
$$

The above formula can be solved for $V$, obtaining

$$
V=\frac{2 C^{2}}{(\ln (\zeta K))^{2}}
$$


where $\zeta \equiv 1 / 2 \alpha$ is a parameter with dimensions of length, and where we assume $K>1 / \zeta$. Differentiating the above equation, we get

$$
-\frac{1}{K} \frac{d V}{d K}=\frac{4 C^{2}}{K^{2}(\ln (\zeta K))^{3}} .
$$

So the expected power spectral density is

$$
\left\langle\tau_{\mathrm{rms}}^{2}\left(K_{0}, K_{1}\right)\right\rangle \propto-\frac{1}{K} \frac{d V}{d K} \propto \frac{1}{K^{2}(\ln (\zeta K))^{3}} .
$$

Notice that this agrees with the Gaussian formula, except that it has $(\ln (\zeta K))^{3}$ in place of $(\ln (\zeta K))^{2}$. The fact that the two formulas are so similar helps give confidence in our use of the Gaussian formula.

\section{Calculation Assuming Exponential Distribution}

Here we assume that each $X_{K}$ is an exponential random variable, with zero mean and variance $V=V(K)$. The exponential distribution is a single-tailed distribution, with probability density proportional to $\exp (-\lambda t)$. By comparison, the Gaussian distribution is a symmetric double-tailed distribution with a probability density proportional to $\exp \left(-t^{2} / 2 \sigma^{2}\right)$. So this calculation explores the effect of using a distribution that falls off more slowly than Gaussian, as well as the effect of using an asymmetric distribution. (Physically, an asymmetric distribution might be interpreted as saying that the stress cannot be much lower than its mean value, but can be much higher than its mean value.)

The exponential distribution (shifted to have zero mean) is defined to have the cumulative density function

$$
P\left(X_{K} \geq C\right)=e^{-1-C / \sqrt{V}} \quad \text { assuming } C \geq-\sqrt{V} .
$$

The restriction on $C$ is not a problem, because $C$ is the threshold stress for defining positive stress perturbations, so physically we will always have $C \geq 0$.

Our scaling relation $P\left(X_{K} \geq C\right)=\alpha / K$ becomes

$$
\frac{\alpha}{K}=e^{-1-C / \sqrt{V}} .
$$

The above formula can be solved for $V$, obtaining

$$
V=\frac{C^{2}}{(\ln (\zeta K))^{2}}
$$

where $\zeta \equiv 1 / e \alpha$ is a parameter with dimensions of length, and where we assume $K>1 / \zeta$. Differentiating the above equation, we get 


$$
-\frac{1}{K} \frac{d V}{d K}=\frac{2 C^{2}}{K^{2}(\ln (\zeta K))^{3}} .
$$

So the expected power spectral density is

$$
\left\langle\tau_{\mathrm{rms}}^{2}\left(K_{0}, K_{1}\right)\right\rangle \propto-\frac{1}{K} \frac{d V}{d K} \propto \frac{1}{K^{2}(\ln (\zeta K))^{3}} .
$$

This is the same formula obtained with the Laplace distribution.

\section{Calculation Assuming Pareto Distribution}

Here we assume that each $X_{K}$ is a Pareto random variable, with zero mean and variance $V=V(K)$. The Pareto distribution is a single-tailed distribution, with probability density proportional to a power law $t^{-(\gamma+1)}$. We require $\gamma>2$ for the Pareto distribution to have finite variance. So, this calculation explores the effect of using a distribution that falls off much more slowly than Gaussian.

The Pareto distribution (shifted to have zero mean) is defined to have the cumulative density function

$$
\begin{aligned}
& P\left(X_{K} \geq C\right)=\left[\sqrt{\frac{(\gamma-1)^{2}(\gamma-2)}{\gamma}} \frac{\sqrt{V}}{\sqrt{\gamma(\gamma-2)} \sqrt{V}+C}\right]^{\gamma} \\
& \text { with } \quad C \geq-\sqrt{\frac{(\gamma-1)^{2}(\gamma-2)}{\gamma}} \sqrt{V} .
\end{aligned}
$$

The restriction on $C$ is not a problem, because $C$ is the threshold stress for defining positive stress perturbations, so physically we will always have $C \geq 0$.

Our scaling relation $P\left(X_{K} \geq C\right)=\alpha / K$ becomes

$$
\frac{\alpha}{K}=\left[\sqrt{\frac{(\gamma-1)^{2}(\gamma-2)}{\gamma}} \frac{\sqrt{V}}{\sqrt{\gamma(\gamma-2)} \sqrt{V}+C}\right]^{\gamma} .
$$

The above formula can be solved for $V$, obtaining

$$
V=C^{\prime 2}\left[\left(\frac{K}{\alpha^{\prime}}\right)^{1 / \gamma}-1\right]^{-2}
$$

where we define

$$
C^{\prime} \equiv \frac{C}{\sqrt{\gamma(\gamma-2)}}
$$




$$
\alpha^{\prime} \equiv \alpha\left(\frac{\gamma}{\gamma-1}\right)^{\gamma}
$$

Differentiating the above equation, we get

$$
-\frac{1}{K} \frac{d V}{d K}=\frac{2 C^{\prime 2}}{\gamma \alpha^{\prime 2}}\left[\left(\frac{K}{\alpha^{\prime}}\right)^{1 / \gamma}-1\right]^{-3}\left(\frac{K}{\alpha^{\prime}}\right)^{1 / \gamma-2}
$$

and so

$$
-\frac{1}{K} \frac{d V}{d K} \sim \frac{2 C^{\prime 2}}{\gamma \alpha^{\prime 2}}\left(\frac{K}{\alpha^{\prime}}\right)^{-2 / \gamma-2} \quad \text { as } K \rightarrow \infty
$$

So the expected power spectral density is

$$
\left\langle\tau_{\mathrm{rms}}^{2}\left(K_{0}, K_{1}\right)\right\rangle \propto-\frac{1}{K} \frac{d V}{d K} \propto \frac{1}{K^{2+2 / \gamma}} \quad \text { as } K \rightarrow \infty .
$$

Recalling that we need $\gamma>2$ for the Pareto distribution to have finite variance, we see that the exponent satisfies $2<2+2 / \gamma<3$. So the asymptotic falloff of the expected power spectral density is a power law that is faster than $1 / K^{2}$, but slower than $1 / K^{3}$.

\section{Technical Issues for One-Point Statistics}

Here we discuss some technical issues relating to the definition of $X_{K}$ and the formulas for its mean and variance. Our goals are to (1) define the concept of a random variable that has the expected one-point statistics of the filtered stress distribution; (2) show that its mean is zero; and (3) show that its variance equals the expected mean-square filtered stress.

\section{Removing Infinities}

We introduced periodic boundary conditions to make the fault area finite. This avoids a number of mathematical and conceptual difficulties. For example, a finite fault makes it straightforward to talk about the mean-square value of the stress or the total elastic energy of a displacement field.

Another advantage of periodic boundary conditions is that it makes the wave vector assume discrete values. But there are still an infinite number of wave vectors. To pick a stress distribution at random, one must (in theory) pick an infinite number of wave amplitudes. This creates some difficulties. For example, the resulting stress distribution would not be a continuous function (it would instead be a so-called $L^{2}$ function); it is not straightforward to define the value of the stress at some given point on the fault; and it is difficult to work with an infinite number of random variables.

We can remove the infinity by imposing a maximum wavenumber $K_{\max }$. That is, we construct our stress field by summing only plane waves with $\left|k_{0}\right| \leq K_{\max }$ and $\left|k_{1}\right| \leq K_{\max }$. The limit $K_{\max }$ is treated as arbitrarily large, much larger than any wavenumber of interest. With this 
constraint, there are only a finite number of admissible wave vectors $\left(k_{0}, k_{1}\right)$, so a stress distribution can be chosen by selecting a finite number of wave amplitudes. As a result, the set of possible stress fields is a finite-dimensional vector space, the stress fields are continuous, and the value of stress at a given point is completely well-defined.

The maximum wavenumber $K_{\max }$ is analogous to the fault dimensions $L_{0}$ and $L_{1}$, in that it is a large number introduced to eliminate infinities from the calculation, with the expectation that the final result does not depend on its value so long as the value is very large.

(Technical remark: We impose the conditions $\left|k_{0}\right| \leq K_{\max }$ and $\left|k_{1}\right| \leq K_{\max }$, instead of the condition $k \leq K_{\max }$, because the former condition guarantees that two-dimensional Fourier transforms converge pointwise in the limit $K_{\max } \rightarrow \infty$ (Fefferman, 1971), whereas it is unknown if the latter condition does so. For our purposes, the exact form of the wavenumber cutoff is not important.)

\section{Stress Distributions and Wave Amplitudes}

Let $\tau_{\text {total }}\left(x_{0}, x_{1}\right)$ denote the shear stress on the fault, which we presume is generated by some random process. Due to the existence of a maximum wavenumber, the stress can be expressed as a finite sum of cosines and sines:

$$
\tau_{\text {total }}\left(x_{0}, x_{1}\right)=\sum_{k_{0}, k_{1}} Z_{C}\left(k_{0}, k_{1}\right) \cos \left(k_{0} x_{0}+k_{1} x_{1}\right)+\sum_{k_{0}, k_{1}} Z_{S}\left(k_{0}, k_{1}\right) \sin \left(k_{0} x_{0}+k_{1} x_{1}\right)
$$

Recall from the main report (equation 18) that we do not include any terms with zero wave vector, $\left(k_{0}, k_{1}\right)=(0,0)$, because a uniform displacement field generates no stress and no elastic energy. Physically, this means that $\tau_{\text {total }}\left(x_{0}, x_{1}\right)$ is the deviation in stress from some unspecified mean stress value.

Let $T$ denote the set of possible wave amplitudes $\left(Z_{C}, Z_{S}\right)$. Then $T$ is a vector space of finite dimension $N$, and each point in $T$ corresponds to a stress distribution according to the above formula.

To randomly select a stress distribution, all that is needed is to choose $N$ random numbers $\left(Z_{C}, Z_{S}\right)$, that is, to choose a point in $T$. We assume that the random selection is made in accordance with a probability density function $\rho\left(Z_{C}, Z_{S}\right)$, which is a non-negative measurable function with an integral of 1.

(Informally, a function is called measurable if it is sufficiently well-behaved so that it can be integrated. Rigorous mathematical definitions may be found in, for example, Royden (1968).)

If $f$ is a real-valued measurable function on $T$ and, hence, also a real-valued function on the set of possible stress distributions, then the expected value of $f$ is

$$
\langle f\rangle \equiv \int_{T} f\left(Z_{C}, Z_{S}\right) \rho\left(Z_{C}, Z_{S}\right) d Z_{C} d Z_{S}
$$

The subscript $T$ emphasizes that the integral is over the set $T$ of wave amplitudes, or stress fields.

Recall that $\tau_{\text {total }}\left(x_{0}, x_{1} ; k \geq K\right)$ denotes the portion of the stress field due to wavenumbers $\geq K$. In other words, it is the result of applying a high-pass filter to $\tau_{\text {total }}\left(x_{0}, x_{1}\right)$, where the filter only passes wavenumbers $\geq K$. (Of course, we assume $K \ll K_{\max }$.) Then 
$\tau_{\text {total }}\left(x_{0}, x_{1} ; k \geq K\right)$ has the same expansion as a sum of cosines and sines, with the same amplitudes, except omitting any terms with $k_{0}^{2}+k_{1}^{2}<K^{2}$. So, we have

$$
\begin{aligned}
\tau_{\text {total }}\left(x_{0}, x_{1} ; k \geq K\right) & \equiv \sum_{k_{0}^{2}+k_{1}^{2} \geq K^{2}} Z_{C}\left(k_{0}, k_{1}\right) \cos \left(k_{0} x_{0}+k_{1} x_{1}\right) \\
& +\sum_{k_{0}^{2}+k_{1}^{2} \geq K^{2}} Z_{S}\left(k_{0}, k_{1}\right) \sin \left(k_{0} x_{0}+k_{1} x_{1}\right) .
\end{aligned}
$$

\section{Fault Surface and Mean Values}

Let $S$ denote the fault surface. Then $S$ consists of points $\left(x_{0}, x_{1}\right)$, subject to the periodic boundary conditions. The fault surface has a finite area, which is $L_{0} L_{1}$.

By Parseval's theorem, the mean-square value of the stress field $\tau_{\text {total }}\left(x_{0}, x_{1}\right)$ equals onehalf the sum of the wave amplitudes:

$$
\tau_{\text {total }}^{2} \equiv \frac{1}{L_{0} L_{1}} \int_{S}\left(\tau_{\text {total }}\left(x_{0}, x_{1}\right)\right)^{2} d x_{0} d x_{1}=\frac{1}{2} \sum_{k_{0}, k_{1}}\left(Z_{C}\left(k_{0}, k_{1}\right)\right)^{2}+\frac{1}{2} \sum_{k_{0}, k_{1}}\left(Z_{S}\left(k_{0}, k_{1}\right)\right)^{2}
$$

The subscript $S$ emphasizes that the integral is taken over the fault surface $S$. Likewise, the mean-square value of the filtered stress field $\tau_{\text {total }}\left(x_{0}, x_{1} ; k \geq K\right)$ is

$$
\begin{aligned}
\tau_{\text {total }}^{2}(k \geq K) & \equiv \frac{1}{L_{0} L_{1}} \int_{S}\left(\tau_{\text {total }}\left(x_{0}, x_{1} ; k \geq K\right)\right)^{2} d x_{0} d x_{1} \\
& =\frac{1}{2} \sum_{k_{0}^{2}+k_{1}^{2} \geq K^{2}}\left(Z_{C}\left(k_{0}, k_{1}\right)\right)^{2}+\frac{1}{2} \sum_{k_{0}^{2}+k_{1}^{2} \geq K^{2}}\left(Z_{S}\left(k_{0}, k_{1}\right)\right)^{2}
\end{aligned}
$$

We see that applying the high-pass filter reduces (or leaves unchanged) the mean-square value:

$$
\tau_{\text {total }}^{2}(k \geq K) \leq \tau_{\text {total }}^{2}
$$

If $\xi$ is a real number, define the step function $\theta_{\xi}$ to be

$$
\theta_{\xi}(x)= \begin{cases}0 & \text { if } x<\xi \\ 1 & \text { if } x \geq \xi\end{cases}
$$

Then $\theta_{\xi}$ is a measurable function (although it is not continuous). If $f\left(x_{0}, x_{1}\right)$ is a measurable real-valued function on $S$, then $\theta_{\xi} \circ f$ is also a measurable function. We define:

$$
v\left(f\left(x_{0}, x_{1}\right) \geq \xi\right) \equiv \int_{S} \theta_{\xi} \circ f\left(x_{0}, x_{1}\right) d x_{0} d x_{1}
$$


We say that $v\left(f\left(x_{0}, x_{1}\right) \geq \xi\right)$ is the area of the portion of the fault surface where the condition $f\left(x_{0}, x_{1}\right) \geq \xi$ is true. That is, it is the area of the portion of the fault surface where the function value $f\left(x_{0}, x_{1}\right)$ is greater than or equal to $\xi$.

\section{Product Space}

Let $S \times T$ denote the product space of $S$ and $T$. By definition, an element of $S \times T$ is a tuple $\left(x_{0}, x_{1}, Z_{C}, Z_{S}\right)$ where $\left(x_{0}, x_{1}\right) \in S$ and $\left(Z_{C}, Z_{S}\right) \in T$. Then $S \times T$ is a space with $N+2$ dimensions.

The function $\rho\left(Z_{C}, Z_{S}\right) / L_{0} L_{1}$ is a probability density function on $S \times T$, as shown by:

$$
\begin{aligned}
\frac{1}{L_{0} L_{1}} \int_{S \times T} \rho\left(Z_{C}, Z_{S}\right) d x_{0} d x_{1} d Z_{C} d Z_{S} & =\frac{1}{L_{0} L_{1}} \int_{T}\left[\int_{S} d x_{0} d x_{1}\right] \rho\left(Z_{C}, Z_{S}\right) d Z_{C} d Z_{S} \\
& =\int_{T} \rho\left(Z_{C}, Z_{S}\right) d Z_{C} d Z_{S}=1 .
\end{aligned}
$$

The subscript $S \times T$ emphasizes that the first integral is taken over the product space $S \times T$. It can be evaluated as an iterated integral by Tonelli's Theorem (Royden, theorem 12.20). Selecting a random point $\left(x_{0}, x_{1}, Z_{C}, Z_{S}\right) \in S \times T$ according to the probability density function $\rho\left(Z_{C}, Z_{S}\right) /$ $L_{0} L_{1}$ is equivalent to selecting a random point $\left(x_{0}, x_{1}\right) \in S$ according to the uniform probability distribution, and independently selecting a random set of wave amplitudes $\left(Z_{C}, Z_{S}\right) \in T$ according to the probability density function $\rho\left(Z_{C}, Z_{S}\right)$.

\section{Definition of Expected One-Point Statistics}

Here we define a random variable $X_{K}$, which has the expected one-point statistics of $\tau_{\text {total }}\left(x_{0}, x_{1} ; k \geq K\right)$.

Conceptually, choosing a value of the random variable $X_{K}$ could be done like this: (1) Select a random point $\left(x_{0}, x_{1}\right)$ on the fault surface, according to a uniform probability distribution. (2) Select a random set of wave amplitudes $\left(Z_{C}, Z_{S}\right)$ according to the probability density function $\rho\left(Z_{C}, Z_{S}\right)$. This is equivalent to selecting a random stress field. (3) Let $X_{K}$ be the corresponding value of $\tau_{\text {total }}\left(x_{0}, x_{1} ; k \geq K\right)$, which can be computed from the expansion of $\tau_{\text {total }}\left(x_{0}, x_{1} ; k \geq K\right)$ into a finite sum of cosines and sines.

We want a mathematically precise definition of $X_{K}$. For $K>0$, let $h_{K}$ be a real-valued function on the product space $S \times T$, defined as follows:

$$
h_{K}\left(x_{0}, x_{1}, Z_{C}, Z_{S}\right) \equiv \tau_{\text {total }}\left(x_{0}, x_{1} ; k \geq K\right)
$$

Then $h_{K}$ is a continuous function and, hence, also a measurable function, because $\tau_{\text {total }}\left(x_{0}, x_{1} ; k \geq K\right)$ is the sum of a finite number of cosine waves and sine waves, all of which are continuous functions.

We define the random variable $X_{K}$ to have the probability measure that is induced by the function $h_{K}$. That is, we define

$$
P\left(X_{K} \geq \xi\right) \equiv \frac{1}{L_{0} L_{1}} \int_{S \times T} \theta_{\xi} \circ h_{K}\left(x_{0}, x_{1}, Z_{C}, Z_{S}\right) \rho\left(Z_{C}, Z_{S}\right) d x_{0} d x_{1} d Z_{C} d Z_{S}
$$


where $P\left(X_{K} \geq \xi\right)$ is the probability that $X_{K}$ is greater than the real number $\xi$.

By the Lebesgue Convergence Theorem (Royden, theorem 11.16) we have

$$
\begin{aligned}
& \lim _{\xi \rightarrow-\infty} P\left(X_{K} \geq \xi\right)=1 \\
& \lim _{\xi \rightarrow+\infty} P\left(X_{K} \geq \xi\right)=0
\end{aligned}
$$

which shows that the definition does indeed describe a probability distribution. By Fubini's Theorem (Royden, theorem 12.19), the integral over $S \times T$ can be evaluated using iterated integration:

$$
\begin{aligned}
P\left(X_{K} \geq \xi\right) & =\frac{1}{L_{0} L_{1}} \int_{T}\left[\int_{S} \theta_{\xi} \circ h_{K}\left(x_{0}, x_{1}, Z_{C}, Z_{S}\right) d x_{0} d x_{1}\right] \rho\left(Z_{C}, Z_{S}\right) d Z_{C} d Z_{S} \\
& =\frac{1}{L_{0} L_{1}} \int_{T} v\left(\tau_{\text {total }}\left(x_{0}, x_{1} ; k \geq K\right) \geq \xi\right) \rho\left(Z_{C}, Z_{S}\right) d Z_{C} d Z_{S} \\
& \equiv\left\langle\frac{1}{L_{0} L_{1}} v\left(\tau_{\text {total }}\left(x_{0}, x_{1} ; k \geq K\right) \geq \xi\right)\right\rangle
\end{aligned}
$$

which is just the expected fraction of the fault surface where the value of the filtered stress $\tau_{\text {total }}\left(x_{0}, x_{1} ; k \geq K\right)$ equals or exceeds $\xi$.

The definition of $X_{K}$ has the following consequence (Royden, proposition 15.1). Suppose that $f(\xi)$ is a measurable function, and assume that

$$
\frac{1}{L_{0} L_{1}} \int_{S \times T}\left|f \circ h_{K}\left(x_{0}, x_{1}, Z_{C}, Z_{S}\right)\right| \rho\left(Z_{C}, Z_{S}\right) d x_{0} d x_{1} d Z_{C} d Z_{S}<\infty
$$

(note the absolute value in the integrand). Then the expected value of $f$ with respect to the random variable $X_{K}$ is given by

$$
\langle f(\xi)\rangle_{X_{K}}=\frac{1}{L_{0} L_{1}} \int_{S \times T} f \circ h_{K}\left(x_{0}, x_{1}, Z_{C}, Z_{S}\right) \rho\left(Z_{C}, Z_{S}\right) d x_{0} d x_{1} d Z_{C} d Z_{S}
$$

The subscript $X_{K}$ on the angle bracket denotes the expected value when $\xi$ is chosen at random according to the probability distribution of $X_{K}$. It is not to be confused with unadorned angle brackets, which always denote the expected value when a stress field is chosen at random according to the probability density function $\rho\left(Z_{C}, Z_{S}\right)$. Furthermore, by Fubini's Theorem (Royden, theorem 12.19), the integral over $S \times T$ can be evaluated using iterated integration: 


$$
\begin{aligned}
\langle f(\xi)\rangle_{X_{K}} & =\frac{1}{L_{0} L_{1}} \int_{T}\left[\int_{S} f \circ h_{K}\left(x_{0}, x_{1}, Z_{C}, Z_{S}\right) d x_{0} d x_{1}\right] \rho\left(Z_{C}, Z_{S}\right) d Z_{C} d Z_{S} \\
& =\left\langle\frac{1}{L_{0} L_{1}} \int_{S} f \circ h_{K}\left(x_{0}, x_{1}, Z_{C}, Z_{S}\right) d x_{0} d x_{1}\right\rangle .
\end{aligned}
$$

\section{Mean and Variance of Expected One-Point Statistics}

We now compute the mean and variance of $X_{K}$.

As discussed in the main report, we assume that the expected mean-square stress is finite. This is because the fault has finite strength, and also because infinitely large stresses are not physically realistic. So

$$
\begin{aligned}
\infty & >\left\langle\tau_{\text {total }}^{2}\right\rangle \geq\left\langle\tau_{\text {total }}^{2}(k \geq K)\right\rangle \equiv\left\langle\frac{1}{L_{0} L_{1}} \int\left(\tau_{\text {total }}\left(x_{0}, x_{1} ; k \geq K\right)\right)^{2} d x_{0} d x_{1}\right\rangle \\
& =\left\langle\frac{1}{L_{0} L_{1}} \int_{S}\left(h_{K}\left(x_{0}, x_{1}, Z_{C}, Z_{S}\right)\right)^{2} d x_{0} d x_{1}\right\rangle \\
& \equiv \frac{1}{L_{0} L_{1}} \int_{T}\left[\int_{S}\left(h_{K}\left(x_{0}, x_{1}, Z_{C}, Z_{S}\right)\right)^{2} d x_{0} d x_{1}\right] \rho\left(Z_{C}, Z_{S}\right) d Z_{C} d Z_{S} \\
& =\frac{1}{L_{0} L_{1}} \int_{S \times T}\left(h_{K}\left(x_{0}, x_{1}, Z_{C}, Z_{S}\right)\right)^{2} \rho\left(Z_{C}, Z_{S}\right) d x_{0} d x_{1} d Z_{C} d Z_{S} .
\end{aligned}
$$

In the above equation, it is valid to change the iterated integral into an integral over $S \times T$ by Tonelli's Theorem (Royden, theorem 12.20). We therefore have

$$
\left\langle\xi^{2}\right\rangle_{X_{K}}=\left\langle\frac{1}{L_{0} L_{1}} \int_{S}\left(h_{K}\left(x_{0}, x_{1}, Z_{C}, Z_{S}\right)\right)^{2} d x_{0} d x_{1}\right\rangle=\left\langle\tau_{\text {total }}^{2}(k \geq K)\right\rangle .
$$

By the Holder Inequality (Royden, theorem 11.25 and problem 11.21), we have

$$
\begin{aligned}
& \frac{1}{L_{0} L_{1}} \int_{S \times T}\left|h_{K}\left(x_{0}, x_{1}, Z_{C}, Z_{S}\right)\right| \rho\left(Z_{C}, Z_{S}\right) d x_{0} d x_{1} d Z_{C} d Z_{S} \\
& \quad \leq\left[\frac{1}{L_{0} L_{1}} \int_{S \times T}\left(h_{K}\left(x_{0}, x_{1}, Z_{C}, Z_{S}\right)\right)^{2} \rho\left(Z_{C}, Z_{S}\right) d x_{0} d x_{1} d Z_{C} d Z_{S}\right]^{1 / 2}<\infty .
\end{aligned}
$$

Therefore, 


$$
\begin{aligned}
\langle\xi\rangle_{X_{K}} & =\left\langle\frac{1}{L_{0} L_{1}} \int_{S} Z_{K}\left(x_{0}, x_{1}, Z_{C}, Z_{S}\right) d x_{0} d x_{1}\right\rangle \\
& =\left\langle\frac{1}{L_{0} L_{1}} \int_{S} \tau_{\text {total }}\left(x_{0}, x_{1} ; k \geq K\right) d x_{0} d x_{1}\right\rangle=0 .
\end{aligned}
$$

In the above equation, the final equality to zero is because each $\tau_{\text {total }}\left(x_{0}, x_{1} ; k \geq K\right)$ by construction has zero mean (that is, it consists of cosine and sine waves with no DC component), assuming $K>0$.

Combining the above equations,

$$
\begin{gathered}
\operatorname{mean}\left(\mathrm{X}_{\mathrm{K}}\right)=\langle\xi\rangle_{X_{K}}=0 \\
\operatorname{var}\left(\mathrm{X}_{\mathrm{K}}\right)=\left\langle\xi^{2}\right\rangle_{X_{K}}=\left\langle\tau_{\text {total }}^{2}(k \geq K)\right\rangle .
\end{gathered}
$$

These are the results shown earlier in this appendix. 


\section{Appendix E. Algorithms for Energy Rolloff}

In this appendix we present algorithms for working with the energy rolloff function, and for selecting random energy values in accordance with the Boltzmann distribution that have the desired rolloff.

\section{Energy Rolloff Function}

Recall from the main report (equations 31 and 51) that we defined an energy rolloff function $g(k)$ where $k$ is the scalar wavenumber. For each wave vector $\left(k_{0}, k_{1}\right)$, there is a basis displacement field, and its expected energy is

$$
\left\langle E\left(k_{0}, k_{1}\right)\right\rangle=\frac{1}{\beta} g(k) .
$$

We chose the following functional form for $g(k)$ :

$$
g(k)=\frac{1}{1+(\eta k)^{3}(\log (1+\zeta k))^{2}} .
$$

The constants $\eta$ and $\zeta$ are adjustable parameters with dimensions of length. Generally, we take $\eta=\zeta$ and adjust $\zeta$ to obtain the desired correlation length. Recall that the correlation length is defined to be $\Lambda=2 \pi / \hat{k}$, where $\hat{k}$ is chosen to maximize the value of $g(\hat{k}) \hat{k}^{2}$. The definition implies that $\hat{k}$ is the solution to the equation $(d / d k)\left(g(k) k^{2}\right)=0$. Evaluating the derivative, we obtain the equation

$$
1=(\eta \hat{k})^{3}\left[\frac{1}{2}(\log (1+\zeta \hat{k}))^{2}+(\log (1+\zeta \hat{k})) \frac{\zeta \hat{k}}{1+\zeta \hat{k}}\right]
$$

Given a desired value of the correlation length $\Lambda$, one can substitute $\hat{k}=2 \pi / \Lambda$ and then solve the above equation numerically to obtain suitable values of $\eta$ and $\zeta$. The right-hand side of the above equation is a monotone increasing function of both $\eta$ and $\zeta$, and so the bisection method can be used to find numerical solutions. For example, our computer code lets the user impose a constraint, $\eta=a \zeta+b$, where $a$ and $b$ are user-specified values. Substituting $\eta \rightarrow a \zeta+$ $b$ into the above equation yields an equation for $\zeta$, which can be solved by the bisection method.

Note that if $\eta=\zeta$ then we have $\zeta \approx 0.18021527845 \Lambda$.

In addition to describing the decrease in energy with increasing wavenumber, the energy rolloff function also establishes a relation between the thermodynamic parameter $\beta$ and the expected mean-square stress (equation 34). Given one, the other can be calculated using

$$
\left\langle\tau_{\text {total }}^{2}\right\rangle=\frac{\mu}{4 \pi \beta} \int_{0}^{\infty} g(k) k^{2} d k
$$

Given our formula for $g(k)$, the improper integral can be evaluated numerically by splitting it into the sum of two integrals: 


$$
\begin{aligned}
\int_{0}^{\infty} g(k) k^{2} d k & \\
& =\int_{0}^{a} \frac{k^{2} d k}{1+(\eta k)^{3}(\log (1+\zeta k))^{2}} \\
& +\int_{0}^{1} \frac{\eta^{-3} t^{-2} d t}{\left(a \eta e^{-1+1 / t}\right)^{-3}+\left(\log \left(1+a \zeta e^{-1+1 / t}\right)\right)^{2}}
\end{aligned}
$$

The value of $a$ should be chosen so that $k=a$ lies in the tail of the function $g(k) k^{2}$. In practice, we use $a=\max (10 / \eta, 5 / \zeta)$. The second integral arises from the change of variable $t \equiv$ $(\log (e k / a))^{-1}$. Each of the two integrals on the right-hand side can be evaluated numerically using adaptive Simpson's rule. Note that the integrand in the second integral is finite at $t=0$ despite the appearance of $t^{-2}$ in the numerator.

\section{State Restriction}

For each wave vector $\left(k_{0}, k_{1}\right)$, we impose a maximum allowed amplitude $F_{\max }\left(k_{0}, k_{1}\right)$ for the basis displacement field:

$$
0 \leq F\left(k_{0}, k_{1}\right) \leq F_{\max }\left(k_{0}, k_{1}\right)
$$

Subject to this restriction, the amplitude, $F\left(k_{0}, k_{1}\right)$, and phase, $\Phi\left(k_{0} . k_{1}\right)$, are chosen randomly according to the Boltzmann probability distribution for a system with two degrees of freedom. Each $F_{\max }\left(k_{0}, k_{1}\right)$ is chosen in such a way so that the expected energy satisfies $\left\langle E\left(k_{0}, k_{1}\right)\right\rangle=$ $g(k) / \beta$.

We now develop a method for generating the random amplitudes and phases. Recall the relation (equation 15) between energy and displacement amplitude:

$$
E\left(k_{0}, k_{1}\right)=\frac{1}{2} M\left(k_{0}, k_{1}\right) F\left(k_{0}, k_{1}\right)^{2}
$$

where we define

$$
M\left(k_{0}, k_{1}\right) \equiv \mu L_{0} L_{1}\left(\frac{2 k^{2}}{k^{2}+k_{0}^{2}}\right)^{2} k
$$

We see that imposing a maximum amplitude $F_{\max }\left(k_{0}, k_{1}\right)$ is equivalent to imposing a maximum energy $E_{\max }\left(k_{0}, k_{1}\right)=(1 / 2) M\left(k_{0}, k_{1}\right) F_{\max }\left(k_{0}, k_{1}\right)^{2}$, with the restriction that

$$
0 \leq E\left(k_{0}, k_{1}\right) \leq E_{\max }\left(k_{0}, k_{1}\right)
$$

For convenience we temporarily write $E$ in place of $E\left(k_{0}, k_{1}\right)$, and similarly for other variables. Define

$$
H \equiv 1-e^{-\beta E_{\max }}
$$


or equivalently

$$
E_{\max }=-\frac{1}{\beta} \log (1-H)
$$

Then the partition function is

$$
\begin{aligned}
Z(\beta) & =\int_{0}^{2 \pi} \int_{0}^{F_{\max }} e^{-\beta E} F d F d \Phi \\
& =2 \pi \int_{0}^{F_{\max }} e^{-\beta E} F d F \\
& =\frac{2 \pi}{M} \int_{0}^{E_{\max }} e^{-\beta E} d E \\
& =\frac{2 \pi}{\beta M} H .
\end{aligned}
$$

We changed variables from $F$ to $E$ using $d E=M F d F$. Also note that if we define $F_{C} \equiv F \cos \Phi$ and $F_{S} \equiv F \sin \Phi$, then $d F_{C} d F_{S}=F d F d \Phi$, which justifies the first integral above. The cumulative probability distribution for $E$ is

$$
\begin{aligned}
& P(E \leq C)=\frac{1}{Z(\beta)} \frac{2 \pi}{M} \int_{0}^{C} e^{-\beta E} d E \\
& =\frac{1}{H}\left(1-e^{-\beta C}\right)
\end{aligned}
$$

where $P(E \leq C)$ denotes the probability that the energy $E$ is less than or equal to some value $C$. The implication of the above formula is that if we define

$$
U \equiv \frac{1}{H}\left(1-e^{-\beta E}\right)
$$

or equivalently

$$
E=-\frac{1}{\beta} \log (1-H U)
$$

then $U$ is uniformly distributed between 0 and 1 . This provides a practical method for generating a random value of the energy $E$ in accordance with the Boltzmann distribution: generate a random value $U$ uniformly distributed in $[0,1]$ and apply the above formula to obtain $E$. A random value of the phase $\Phi$ can be generated as $\Phi=2 \pi V$ where $V$ is uniformly distributed in $[0,1]$. The expected energy is obtained by differentiating the partition function (equation 4 ): 


$$
\langle E\rangle=-\frac{1}{Z(\beta)} \frac{d Z}{d \beta}=\frac{1}{\beta}\left[1+\frac{1}{H}(1-H) \log (1-H)\right] .
$$

Notice that $H$ can range from 0 to 1 , and in the process $\langle E\rangle$ ranges from 0 to $1 / \beta$. So, given any desired value of the expected energy $\langle E\rangle$ in the range from 0 to $1 / \beta$, the above equation can be solved numerically to obtain the required value of $H$. Recalling that $\langle E\rangle=$ $g(k) / \beta$, the equation that must be solved to obtain $H$ is:

$$
g(k)=B(H) \equiv 1+\frac{1}{H}(1-H) \log (1-H) .
$$

The equation can be solved using Newton's method, except that the starting value must be carefully chosen. Because $B^{\prime}(H) \rightarrow \infty$ as $H \rightarrow 1$, Newton's method can converge very slowly or fail to converge if the starting value is poor. The following technique can be used to select a proper starting value. Define $H_{1} \equiv 0.683803$. (The value is chosen so that $B^{\prime}\left(H_{1}\right) \approx 1$, noting that $\left.B^{\prime}(0)=1 / 2\right)$. If $g \leq B\left(H_{1}\right)$, then the starting value is:

$$
2 g-\frac{4}{3} g^{2}+\frac{4}{9} g^{3}-\frac{16}{135} g^{4}+\frac{8}{405} g^{5}-\frac{16}{2835} g^{6} .
$$

The above formula contains the first six terms of the power-series expansion of $H$ as a function of $g$.

Otherwise, if $g>B\left(H_{1}\right)$, define an increasing sequence of values by $H_{i+1} \equiv 1-$ $\left(1-H_{i}\right)^{2}$. The starting value for Newton's method is $H_{j}$, where $j$ is the smallest index such that $g \leq B\left(H_{j}\right)$. If $H_{j}$ becomes very close to 1 before the condition $g \leq B\left(H_{j}\right)$ is satisfied (which may occur if $g$ is very close to 1 ), then take $H=1$.

Remark: The starting value works for the following reasons. If $g \leq B\left(H_{1}\right)$, the power series gives a fairly accurate approximation of $H$. For the case $g>B\left(H_{1}\right)$, note that $B^{\prime}(H)>0$ and $B^{\prime \prime}(H)>0$ for all $H$, which implies that convergence of Newton's method is guaranteed provided that the starting value is greater than the true value of $H$. Convergence can be very slow due to the fact that $B^{\prime}(H) \rightarrow \infty$ as $H \rightarrow 1$. But Newton's method converges at least as fast as bisection if the starting value and the true value of $H$ lie within an interval where the derivative changes by a factor of 2 (or less). The sequence $H_{i}$ is constructed so that $B^{\prime}\left(H_{i+1}\right) \approx 2 B^{\prime}\left(H_{i}\right)$, and the starting value is the first value in the sequence that is greater than or equal to the true $H$.

Remark: As $H_{i}$ approaches 1, the recurrence formula $H_{i+1} \equiv 1-\left(1-H_{i}\right)^{2}$ suffers a loss of precision. This can be avoided by writing $S_{i} \equiv 1-H_{i}$, because then the recurrence becomes $S_{i+1}=S_{i}^{2}$ which does not suffer a loss of precision. Note that if we also write $S \equiv 1-H$ then the equation to solve is

$$
g(k)=B(S) \equiv 1+\frac{1}{1-S} S \log S .
$$

To avoid loss of precision, Newton's method can be used to solve this equation for $S$, and then $H$ is obtained as $H=1-S$. $H:$

Remark: In our computer implementation of the method, we impose a maximum value on 


$$
H \leq 1-e^{-5.5 g} .
$$

If the computed $H$ violates the above limit, then $H$ is set equal to its maximum allowed value. This has the effect of limiting the random energy $E$ to a maximum of 5.5 times its expected value. Because the energy follows an exponential distribution, this is equivalent to 5.5 standard deviations above its mean. Note that this limit only comes into play for $g \gtrsim 0.975$, and so it affects only long-wavelength (small wavenumber) Fourier modes where the energy rolloff $g(k)$ is close to 1 . If this was not done, then the random stress pattern could be dominated by a small number of long-wavelength Fourier modes with extremely large amplitudes. We consider this to be a form of discretization error and adopt the above limit to prevent it. (There is nothing special about the value 5.5; there is a range of values that would work as well.) 


\section{Appendix F. Mathematics of the Selection Algorithm}

In this appendix we explain the mathematics behind the selection algorithm. As explained in the main report, the purpose of the selection algorithm is to select the fault surface from a much larger distribution of randomly generated stresses.

Recall (from equations 58 and 59) that the large stress distribution is defined on an $N_{0} \times N_{1}$ grid of points $\left(x_{0}, x_{1}\right)$ :

$$
\begin{array}{ll}
x_{0}=\left(m_{0} / N_{0}\right) L_{0} & \text { for } 0 \leq m_{0}<N_{0} \\
x_{1}=\left(m_{1} / N_{1}\right) L_{1} & \text { for } 0 \leq m_{1}<N_{1}
\end{array}
$$

where $m_{0}$ and $m_{1}$ are integers, and where $L_{0}$ and $L_{1}$ are the physical size of the grid in the $x_{0}$ and $x_{1}$ directions. The grid is considered to satisfy periodic boundary conditions. Because the points $\left(x_{0}, x_{1}\right)$ are discrete, a function $f\left(x_{0}, x_{1}\right)$ is nothing more than $N_{0} N_{1}$ function values, and so may be regarded as an element of an $N_{0} N_{1}$-dimensional vector space. Given two functions, $f_{1}\left(x_{0}, x_{1}\right)$ and $f_{2}\left(x_{0}, x_{1}\right)$, we can calculate their dot product within this vector space:

$$
f_{1} \cdot f_{2} \equiv \sum_{y_{0} y_{1}} f_{1}\left(y_{0}, y_{1}\right) f_{2}\left(y_{0}, y_{1}\right)
$$

The sum runs over the $N_{0} N_{1}$ possible values of $\left(y_{0}, y_{1}\right)$ and is nothing more than the usual dot product in a slightly unusual notation.

The dot product can be used as a measure of the extent to which two vectors point in the same direction. It is an imperfect measure, but in general, a large positive dot product indicates a likelihood that the two vectors point in roughly the same direction. Expressed in the language of functions, a large positive dot product indicates a likelihood that the two functions are similar.

\section{The Convolution}

Recall from the main report (equation 72) that the complex-valued randomly generated stress field is

$$
\tau_{\text {total }}^{\prime}\left(x_{0}, x_{1}\right)=-\sum_{k_{0} k_{1}} \sqrt{\frac{2 \mu E\left(k_{0}, k_{1}\right) k}{L_{0} L_{1}}} \exp \left(i k_{0} x_{0}+i k_{1} x_{1}+i \Phi\left(k_{0}, k_{1}\right)\right)
$$

where $k_{0}$ and $k_{1}$ are wavenumbers in the $x_{0}$ and $x_{1}$ directions, $E\left(k_{0}, k_{1}\right)$ are randomly selected energies, and $\Phi\left(k_{0}, k_{1}\right)$ are randomly selected phases.

We are given a real-valued selection template $Q\left(x_{0}, x_{1}\right)$ that describes the desired pattern of stress on the fault surface, under the assumption that the upper left corner of the fault surface is aligned at the origin $\left(x_{0}, x_{1}\right)=(0,0)$. Then, we form the convolution (equation 73 ):

$$
\left(Q \star \tau_{\text {total }}^{\prime}\right)\left(x_{0}, x_{1}\right) \equiv \sum_{y_{0} y_{1}} Q\left(y_{0}, y_{1}\right) \tau_{\text {total }}^{\prime}\left(x_{0}+y_{0}, x_{1}+y_{1}\right)
$$


For each $\left(x_{0}, x_{1}\right)$, the sum on the right-hand side is a dot product of two functions. One function is the stress, $\tau_{\text {total }}^{\prime}$. The other function is the selection template, $Q$, shifted so that the upper left corner of the fault surface lies at the point $\left(x_{0}, x_{1}\right)$. To see that the selection template is shifted in this way, note that for $\left(y_{0}, y_{1}\right)=(0,0)$ the term appearing in the sum is $Q(0,0) \tau_{\text {total }}^{\prime}\left(x_{0}, x_{1}\right)$, which shows that the origin of the selection template is aligned with point $\left(x_{0}, x_{1}\right)$ of the stress field.

So if we take $\left(\tilde{x}_{0}, \tilde{x}_{1}\right)$ to be the point which maximizes the absolute value of the convolution, then $\left(\tilde{x}_{0}, \tilde{x}_{1}\right)$ is the point where we should place the upper left corner of the fault surface so as to maximize the absolute value of the dot product of the stress field with the selection template.

\section{The Phase Shift}

Recall from the main article (equation 74) that $\theta$ is the complex phase of the convolution, $\left(Q \star \tau_{\text {total }}^{\prime}\right)\left(\tilde{x}_{0}, \tilde{x}_{1}\right)$, at the point where its absolute value is maximized, that is,

$$
\left(Q \star \tau_{\text {total }}^{\prime}\right)\left(\tilde{x}_{0}, \tilde{x}_{1}\right)=\left|\left(Q \star \tau_{\text {total }}^{\prime}\right)\left(\tilde{x}_{0}, \tilde{x}_{1}\right)\right| \cdot e^{i \theta}
$$

Now define

$$
\tau^{\prime}\left(x_{0}, x_{1}\right) \equiv \tau_{\text {total }}^{\prime}\left(x_{0}, x_{1}\right) \cdot e^{-i \theta}
$$

Then

$$
\left(Q \star \tau^{\prime}\right)\left(\tilde{x}_{0}, \tilde{x}_{1}\right)=\left(Q \star \tau_{\text {total }}^{\prime}\right)\left(\tilde{x}_{0}, \tilde{x}_{1}\right) \cdot e^{-i \theta}=\left|\left(Q \star \tau_{\text {total }}^{\prime}\right)\left(\tilde{x}_{0}, \tilde{x}_{1}\right)\right|
$$

In other words, $\left(Q \star \tau^{\prime}\right)\left(\tilde{x}_{0}, \tilde{x}_{1}\right)$ is real and positive. In fact, it is the largest positive value that can be obtained by considering any point in the stress field and any phase. Now define

$$
\begin{aligned}
\tau\left(x_{0}, x_{1}\right) & \equiv \operatorname{Re}\left(\tau^{\prime}\left(x_{0}, x_{1}\right)\right) \\
& =-\sum_{k_{0} k_{1}} \sqrt{\frac{2 \mu E\left(k_{0}, k_{1}\right) k}{L_{0} L_{1}}} \cos \left(k_{0} x_{0}+k_{1} x_{1}+\Phi\left(k_{0}, k_{1}\right)-\theta\right)
\end{aligned}
$$

which is the phase-shift formula from the main report (equation 75). Because the selection template $Q$ is real, we have

$$
(Q \star \tau)\left(\tilde{x}_{0}, \tilde{x}_{1}\right)=\operatorname{Re}\left(\left(Q \star \tau^{\prime}\right)\left(\tilde{x}_{0}, \tilde{x}_{1}\right)\right)=\left|\left(Q \star \tau_{\text {total }}^{\prime}\right)\left(\tilde{x}_{0}, \tilde{x}_{1}\right)\right|
$$

So we have also maximized the value of $Q \star \tau$. This means that the phase-shifted stress field $\tau\left(x_{0}, x_{1}\right)$ is likely to be similar to the location-shifted selection template, with the shift chosen to place the upper left corner of the fault surface at the point $\left(\tilde{x}_{0}, \tilde{x}_{1}\right)$. Indeed, the chosen point $\left(\tilde{x}_{0}, \tilde{x}_{1}\right)$ and phase $\theta$ are likely to maximize the degree of similarity. 


\section{Appendix G. Post-Processing}

In this appendix we describe post-processing steps that can be used to prepare the random stress distribution for use in a dynamic rupture simulation.

The main report describes how to construct a raw random stress field on a fault surface. Before the stress field can be used as input to a dynamic rupture simulation, it is generally necessary to perform some post-processing steps. The steps required will depend on the details of the model, such as the friction parameters, the variation of normal stress with depth, and the characteristics of the simulation software. Here we describe typical post-processing steps that may be used, in the order that they are applied.

\section{Stress Field Rotation}

Recall that the random stress distribution on the fault surface is cut out from a much larger stress distribution. The cut-out portion is selected by finding the portion of the large stress distribution that is most similar to a user-specified selection template $Q\left(x_{0}, x_{1}\right)$, where $x_{0}$ and $x_{1}$ are the along-dip and along-strike coordinates on the fault surface. See the main report (equation 73) for details.

If the selection template $Q\left(x_{0}, x_{1}\right)$ has symmetry under rotation by 180 degrees, as is the case for the selection template shown in figure 8 , then it is possible to rotate the stress field by 180 degrees around the center of symmetry of the selection template. The effect of performing such a rotation is that the stress pattern, which is originally near the Earth's surface, is moved to the bottom of the fault and vice versa. (Note that if the center of symmetry of the selection template does not coincide with the center of symmetry of the fault surface, then performing the rotation also causes a slightly different portion of the large stress distribution to be used when the fault surface is cut out.)

For many dynamic rupture simulations, particularly in cases where the normal stress increases with depth, high stress near the bottom of the fault is more effective at driving the rupture than high stress near the Earth's surface. So, one can perform a test to check if the original stress field has more high stress in the upper part of the fault than in the lower part and, if so, rotate the stress field by 180 degrees.

To test whether or not to perform the rotation, we calculate

$$
\sum_{x_{0} x_{1}} R\left(x_{0}, x_{1}\right) \tau\left(x_{0}, x_{1}\right)
$$

where the sum runs over all points $\left(x_{0}, x_{1}\right)$ on the fault surface, $R\left(x_{0}, x_{1}\right)$ is a user-specified function called the rotation template, and $\tau\left(x_{0}, x_{1}\right)$ is the random shear stress. The above sum is calculated for both the rotated and un-rotated stress fields, and whichever one has a larger sum is used. The rotation template $R\left(x_{0}, x_{1}\right)$ is typically chosen to be zero at the Earth's surface, and positive near the bottom of the fault, so that stresses at depth contribute more to the sum than stresses near the Earth's surface.

\section{Filtering}

Simulation codes typically cannot resolve details smaller than 3 to 5 grid points. Our randomly generated stress field contains details down to the level of individual grid points. It is 
desirable to suppress such details, so as not to exceed the resolution of the simulation code. We do this by applying a weighted moving-average filter to the stress field:

$$
\tau\left(x_{0}, x_{1}\right) \leftarrow \sum_{m_{0}=-M_{0}}^{M_{0}} \sum_{m_{1}=-M_{1}}^{M_{1}} c\left(m_{0}, m_{1}\right) \tau\left(x_{0}+m_{0} h_{0}, x_{1}+m_{1} h_{1}\right)
$$

where $h_{0}$ and $h_{1}$ are the spacing between adjacent points on the fault surface. The coefficients $c\left(m_{0}, m_{1}\right)$ are called the filter kernel. We take them to be bilinear, which is defined as:

$$
c\left(m_{0}, m_{1}\right)=C\left(M_{0}+1-\left|m_{0}\right|\right)\left(M_{1}+1-\left|m_{1}\right|\right)
$$

The constant $C$ is chosen so that

$$
\sum_{m_{0}=-M_{0}}^{M_{0}} \sum_{m_{1}=-M_{1}}^{M_{1}} c\left(m_{0}, m_{1}\right)=1
$$

But the choice of filter kernel is not important. The moving-average filter acts as a lowpass spatial filter. Although it can be evaluated using Fourier transforms, it is typically better to just evaluate the summation directly, because typically $M_{0}$ and $M_{1}$ are quite small. For the examples included with this report, we used $M_{0}=M_{1}=2$.

An issue with a moving-average filter is what to do near the edges of the fault surface, where the filter extends outside the fault surface. In our case, the fault surface is cut out from a much larger stress distribution. So stress values outside the fault surface are available, and we use them when applying the filter near the edges of the fault surface.

\section{Scaling}

To perform dynamic rupture simulations, the stresses must be scaled to be consistent with the friction law. In the high-stress (center) portion of the fault surface, the stresses must be high enough to sustain the rupture but below the fault's yield stress. In the low-stress (side and bottom) portion of the fault surface, the stresses must be low enough to stop the rupture.

Our recommended procedure is to generate a group of 10 or 20 stress distributions, and then scale all of them at once. Then the scaling formula is:

$$
\tau_{n}\left(x_{0}, x_{1}\right) \leftarrow a \cdot \tau_{n}\left(x_{0}, x_{1}\right)+b_{n}
$$

where $\tau_{n}$ is the $n$th stress field, and the numbers $a$ and $b_{n}$ are selected as needed to make the stress field consistent with the friction law. Notice that the same scale factor $a$ is used for all the stress distributions in the group; this allows the amplitude of the stress fluctuations to vary among the stress realizations.

After scaling, the stress field may exceed the fault's yield stress at some points. If this occurs, then at those points either the stress field should be reduced, or the friction parameters should be modified, to increase the yield stress.

See the example at the end of this appendix for further discussion of scaling. 


\section{Hypocenter Selection}

When heterogeneous initial stresses are used, the hypocenter should be located within a region of relatively high initial stress.

In our computer code, the user specifies a range of permissible hypocenter locations, along-strike and along-dip. For each permissible location $\left(x_{0}, x_{1}\right)$, the code calculates a weighted average of the initial stresses at nearby points, like this:

$$
\sum_{m_{0}=-M_{0}}^{M_{0}} \sum_{m_{1}=-M_{1}}^{M_{1}} d\left(m_{0}, m_{1}\right) \tau\left(x_{0}+m_{0} h_{0}, x_{1}+m_{1} h_{1}\right)
$$

where $h_{0}$ and $h_{1}$ are the spacing between adjacent points on the fault surface, and where $\tau$ is the shear stress (after scaling). The coefficients $d\left(m_{0}, m_{1}\right)$ are called the hypocenter selection mask. We typically take them to be Gaussian, which is defined as:

$$
d\left(m_{0}, m_{1}\right)=D \exp \left(-\frac{1}{2}\left(\left(m_{0} / s_{0}\right)^{2}+\left(m_{1} / s_{1}\right)^{2}\right)\right)
$$

The constant $D$ is chosen so that

$$
\sum_{m_{0}=-M_{0}}^{M_{0}} \sum_{m_{1}=-M_{1}}^{M_{1}} d\left(m_{0}, m_{1}\right)=1
$$

Typically we take $M_{0}=M_{1}=10$ and $s_{0}=s_{1}=7$. However, the precise form of the hypocenter selection mask is not important.

The location $\left(x_{0}, x_{1}\right)$ on the fault with the highest weighted-average nearby stress is chosen as the hypocenter.

\section{Size of Nucleation Zone}

The main report describes a new nucleation method, which combines a small zone of forced rupture (equation 77) and a larger zone of reduced fracture energy (equation 78). Both zones are circles centered at the hypocenter. If this method is to be used, then it is necessary to select the radius $R_{\mathrm{ZRFE}}$ of the zone of reduced fracture energy. (The radius of the zone of forced rupture is then $R_{\mathrm{ZFR}}=R_{\mathrm{ZRFE}} / 4$.)

We choose the radius of the zone of reduced fracture energy to be

$$
R_{\mathrm{ZRFE}}=F_{\mathrm{NUKE}} R_{D}
$$

where $R_{D}$ is the Day radius (equation 76), and $F_{\mathrm{NUKE}}$ is a user-specified parameter called the nucleation factor. The nucleation factor is chosen to be large enough, so that the nucleation method operates reliably. In our tests, we find that $F_{\text {NUKE }}$ values in the range 1.00 to 1.25 work well.

Some experimentation may be necessary to select an appropriate value of $F_{\mathrm{NUKE}}$, by running dynamic rupture simulations with different values of $F_{\mathrm{NUKE}}$. If $F_{\mathrm{NUKE}}$ is too small, the 
simulated rupture will die out before exiting the nucleation zone, or shortly after exiting the nucleation zone. If $F_{\mathrm{NUKE}}$ is just slightly too small, the simulated rupture may slow down or stop near the edge of the nucleation zone, then speed up and continue after a delay. When $F_{\mathrm{NUKE}}$ is large enough, then the rupture should propagate smoothly through the nucleation zone and on into the surrounding fault. On the other hand, $F_{\mathrm{NUKE}}$ should not be excessively large, because we desire to minimize the effect of the nucleation process on the rupture propagation. By performing several dynamic rupture simulations, with several different values of $F_{\mathrm{NUKE}}$, and observing the resulting simulated rupture propagation, it is possible to select an appropriate value of $F_{\mathrm{NUKE}}$.

The Day radius (Day, 1982) is defined to be

$$
R_{D}=\frac{7 \pi \mu\left(\tau_{s}-\tau_{d}\right) D_{c}}{24\left(\tau-\tau_{d}\right)^{2}}
$$

Here, $\mu$ is the shear modulus, $\tau$ is the initial shear stress, $\tau_{s}$ is the static yield stress, $\tau_{d}$ is the dynamic sliding stress, and $D_{c}$ is the slip-weakening critical distance, that is, the amount of slip over which the frictional stress declines from $\tau_{s}$ to $\tau_{d}$. The fracture energy per unit area is $\left(\tau_{s}-\tau_{d}\right) D_{c} / 2$

To compute the Day radius, we use the approximation

$$
R_{D} \approx \frac{7 \pi \mu\left(\mu_{s}-\mu_{d}\right) D_{c}}{24\left(\left(\tau_{\mathrm{avg}} / \sigma_{n}\right)-\mu_{d}\right)^{2} \sigma_{n}}
$$

Here, $\mu$ is the shear modulus in the neighborhood of the hypocenter, $\mu_{s}$ is the static coefficient of friction at the hypocenter, $\mu_{d}$ is the dynamic coefficient of friction at the hypocenter, $D_{c}$ is the slip-weakening critical distance at the hypocenter (not taking into account the reduced critical distance used for nucleation), and $\sigma_{n}$ is the initial normal stress at the hypocenter. The fraction $\tau_{\text {avg }} / \sigma_{n}$ is the average ratio of initial shear stress to initial normal stress, over a user-specified rectangular portion of the fault surface. Because of the scaling by normal stress, which is described below, $\tau_{\text {avg }} / \sigma_{n}$ is actually proportional to the "initial stress" value at this stage of the post-processing.

Note that this calculation implicitly assumes the use of a slip-weakening friction law. Such a law is characterized by a static coefficient of friction $\mu_{s}$, a dynamic coefficient of friction $\mu_{d}$, and a slip-weakening critical distance $D_{c}$, which is defined to be the amount of fault slip over which the coefficient of friction decreases from $\mu_{s}$ to $\mu_{d}$.

\section{Depth Conditioning}

If the goal is to model earthquakes large enough to rupture the full depth of the seismogenic zone, then depth conditioning is used to limit rupture extent at the bottom of the fault. This is appropriate because the base of the seismogenic zone is defined by a systematic change in the properties of the fault, such as a transition from brittle to ductile behavior.

Depth conditioning systematically reduces the shear stress with increasing depth. The shear stress is multiplied by a depth-conditioning function, $\Omega$, which varies as a function of depth (equation 69):

$$
\tau\left(x_{0}, x_{1}\right) \leftarrow \tau\left(x_{0}, x_{1}\right) \Omega\left(x_{0}\right)
$$


In the examples shown here, the depth conditioning function is taken to be piecewise linear (equation 70):

$$
\Omega\left(x_{0}\right)=\left\{\begin{aligned}
1.00 & \text { for } 0 \geq x_{0} \geq-14,000 \mathrm{~m} \\
0.80+0.0001\left(x_{0}+16,000 \mathrm{~m}\right) & \text { for }-14,000 \mathrm{~m} \geq x_{0} \geq-16,000 \mathrm{~m} \\
0.10+0.0002\left(x_{0}+19,500 \mathrm{~m}\right) & \text { for }-16,000 \mathrm{~m} \geq x_{0} \geq-19,500 \mathrm{~m}
\end{aligned}\right.
$$

It is of course possible to make the depth conditioning function be smooth rather than piecewise linear.

If the goal is to model smaller earthquakes that do not rupture the full depth of the seismogenic zone, then depth conditioning should not be used. In this case, the selection template is used to control rupture extent both vertically and horizontally.

\section{Scaling by Normal Stress}

A dynamic rupture simulation will assume some pattern of initial normal stress on the fault. In general, friction forces are proportional to the normal stress. So, to remain consistent with the friction law, we scale the shear stress by a factor proportional to the normal stress:

$$
\tau\left(x_{0}, x_{1}\right) \leftarrow w \cdot \sigma\left(x_{0}, x_{1}\right) \cdot \tau\left(x_{0}, x_{1}\right)
$$

where $\sigma\left(x_{0}, x_{1}\right)$ is the initial normal stress on the fault at point $\left(x_{0}, x_{1}\right)$, and where $w$ is some constant chosen to attain consistency with the friction law.

Another way of saying this is that we treat our randomly generated values as if they lie in "friction coefficient space."

\section{Example of Scaling}

We present an extended example of scaling, to illustrate the group scaling (equation 289) and normal scaling (equation 298) procedures. The scaling technique described here was used in the examples that accompany this report. We emphasize that this is an example, that there are other acceptable ways to perform the scaling, and that, in any event, the scaling must be adjusted to produce stress fields consistent with the chosen friction parameters.

1. For this example, we assume a slip-weakening friction law, with static coefficient of friction $\mu_{s}=0.677$ and dynamic coefficient of friction $\mu_{d}=0.525$.

2. For this example, we assume the initial normal stress has a constant value $\sigma\left(x_{0}, x_{1}\right)=$ 120 megapascal.

3. Because of the normal stress scaling, the value of $\tau_{n}\left(x_{0}, x_{1}\right)$ appearing on the left side of equation 298 should be regarded as lying in "friction coefficient space," that is, it is the ratio of the initial shear stress divided by the normal stress (and hence its values should lie in the same range as the friction coefficients).

4. Recall that the selection template $Q\left(x_{0}, x_{1}\right)$ (equation 73) specifies high-stress and low-stress regions of the fault surface. In figure 8 , the high-stress region is the deepest red area, and the low-stress region is the deepest blue area. 
5. Let $\bar{\tau}_{n}^{\text {high }}$ denote the average value of $\tau_{n}\left(x_{0}, x_{1}\right)$ in the high-stress region. Let $\bar{\tau}_{n}^{\text {low }}$ denote the average value of $\tau_{n}\left(x_{0}, x_{1}\right)$ in the low-stress region.

6. For this example, we select a target value for $\bar{\tau}_{n}^{\text {high }}$, which is $\mu_{0}^{\text {high }}=0.5835$. However, we do wish to allow some random variation in the value of $\bar{\tau}_{n}^{\text {high }}$, that is, we do not want to force $\bar{\tau}_{n}^{\text {high }}$ to be exactly equal to $\mu_{0}^{\text {high }}$.

If we define the earthquake strength ratio (Day, 1982) to be $S_{n} \equiv\left(\mu_{s}-\bar{\tau}_{n}^{\text {high }}\right) /$ $\left(\bar{\tau}_{n}^{\text {high }}-\mu_{d}\right)$, then the target value of $S_{n}$ is $\left(\mu_{s}-\mu_{0}^{\text {high }}\right) /\left(\mu_{0}^{\text {high }}-\mu_{d}\right)=1.60$ (this is how we selected the numerical value of $\left.\mu_{0}^{\text {high }}\right)$. The earthquake strength ratio is an indicator of how fast the rupture propagates, with lower ratios yielding faster propagation. By allowing some random variation in value of $\bar{\tau}_{n}^{\text {high }}$, we are thereby allowing random variation in $S_{n}$ and hence in the rupture propagation speed. The reason that we employ group scaling (equation 289) is to obtain this random variation in rupture speed.

7. For this example, we select a target value for $\bar{\tau}_{n}^{\text {low }}$, which is $\mu_{0}^{\text {low }}=0.4665$. The value must be chosen far enough below $\mu_{d}$ so that the rupture comes to a stop when it enters the low-stress region. For this example, we have chosen the numerical value of $\mu_{0}^{\text {low }}$ to satisfy $\mu_{0}^{\text {high }}-\mu_{d}=\mu_{d}-\mu_{0}^{\text {low }}$. Again, we want to allow some random variation in the value of $\bar{\tau}_{n}^{\text {low }}$.

8. The parameter $a$ in equation 289 is chosen so that the average value of $\bar{\tau}_{n}^{\text {high }}-\bar{\tau}_{n}^{\text {low }}$ is made equal to $\mu_{0}^{\text {high }}-\mu_{0}^{\text {low }}$.

9. Then, each parameter $b_{n}$ in equation 289 is chosen so that $\frac{1}{2}\left(\bar{\tau}_{n}^{\text {high }}+\bar{\tau}_{n}^{\text {low }}\right)=\mu_{d}$.

10. Then, equation 289 is used to calculate all the values of $\tau_{n}\left(x_{0}, x_{1}\right)$.

11. Then, we adjust the values of $\tau_{n}\left(x_{0}, x_{1}\right)$ so they are not excessively high or low. In this example, we impose the constraint $0.373 \leq \tau_{n}\left(x_{0}, x_{1}\right) \leq 0.677$. Any computed value of $\tau_{n}\left(x_{0}, x_{1}\right)$ that lies outside this range is adjusted to bring it into range.

12. Then, for points within the nucleation zone, adjust the values of $\tau_{n}\left(x_{0}, x_{1}\right)$ so they are above the dynamic coefficient of friction. This is done so that there are no low stresses within the nucleation zone that could interfere with the nucleation process. In this example, we impose the constraint $0.531 \leq \tau_{n}\left(x_{0}, x_{1}\right)$ for points within the nucleation zone. Any computed value of $\tau_{n}\left(x_{0}, x_{1}\right)$ that lies outside this range is adjusted to bring it into range. The value 0.531 was obtained as being approximately $\mu_{d}+0.1\left(\mu_{0}^{\text {high }}-\mu_{d}\right)$.

13. Then, we increase the static coefficient of friction, where necessary, to make the yield stress higher than the initial stress. In this example, we impose the constraint $\mu_{s}-$ $\tau_{n}\left(x_{0}, x_{1}\right) \geq 0.012$. At any point where this constraint is violated, we increase $\mu_{s}$ so the constraint is satisfied (thereby producing a non-uniform value of $\mu_{s}$ on the fault surface).

14. Finally, we compute the actual initial shear stresses by applying equation 298 with the parameter $w$ equal to 1 . 
The end result of this scaling procedure is a heterogeneous initial stress field that is consistent with the chosen friction law. In this example, the initial stress in the high-stress region is consistent with an average strength ratio $S=1.6$; the initial stress in the low-stress region is low enough to arrest the rupture; there is random variability in the strength ratio; the initial stress is everywhere below the yield stress; and the initial stress inside the nucleation zone is above the dynamic sliding stress. 


\section{Appendix H. One-Point Statistics}

In this appendix we present some empirical results on the one-point statistics produced by our method and the effect of applying a low-pass spatial filter to the stress field.

The term one-point statistics refers to the statistical properties of the stress field evaluated at individual points on the fault surface (such as, not taking into account any correlations between stress values at different points on the fault surface). It has been suggested (for example, Lavallee and others, 2006) on the basis of slip inversions that the one-point statistics should be non-Gaussian and, in particular, that it should be more heavy-tailed than a Gaussian. We present evidence that our method produces heavy-tailed one-point statistics.

To assess the one-point statistics, we treat the all the stresses as being values of a random variable and perform a simple test to see if their distribution appears Gaussian or heavy-tailed. To perform the test, we partition the range of stress values into 50 bins. The fraction of stress values that fall into each bin is taken to be an estimate of the probability density function. Then, we find both the best-fitting Gaussian distribution and the best-fitting Cauchy distribution. Note that the Cauchy distribution is so heavy-tailed that its variance is infinite. Then we calculate the ratio $r_{c} / r_{g}$, where $r_{c}$ is the residual from the Cauchy fit, and $r_{g}$ is the residual from the Gaussian fit. With this metric, high values tend to indicate a Gaussian distribution, and low values tend to indicate a heavy-tailed distribution. We performed this calculation for 20 random stress distributions.

Recall that in our method, the stress pattern on the fault surface is cut out from a large stress distribution that is thousands of times larger than the fault surface. If we use stress values from the entire large stress distribution, before the fault surface is cut out, the average ratio is 38.5. This indicates that the one-point statistics of the large stress distribution is very well described as being Gaussian.

If we use stress values from just the selected fault surface, the average ratio is 1.26 if the stress is not low-pass filtered, or 1.23 if the stress is filtered. If we use just the stress values from a rectangular portion of the fault surface that lies in the high-stress area, the average ratio is 1.90 if the stress is not filtered, or 1.85 if the stress is filtered. In either case, the ratios indicate that the one-point statistics are more heavy-tailed than a Gaussian. It is interesting to note that applying a low-pass spatial filter does not alter the heavy-tailed nature of the one-point statistics.

\section{Discussion}

Our method, like some prior methods (for example, Andrews and Barall, 2011), generates stress values by summing large numbers of random values. Such methods have a strong tendency to generate Gaussian one-point statistics, due to the action of the Central Limit Theorem. This is illustrated by the nearly perfect Gaussian one-point statistics that are observed on the large stress distribution.

There has been some question as to whether it is possible for methods of this type to produce heavy-tailed, non-Gaussian statistics. Here we present evidence that this is indeed possible, by selecting just a portion of the generated stress values. Although the entire set of stress values on the large stress distribution is always Gaussian, the stress values on the selected fault surface can have non-Gaussian statistics. 
Table 1. Mathematical notations.

The following table is a list of mathematical notations used in the main portion of this report. (Note that some appendixes may use slightly different notations.)

\begin{tabular}{|c|c|}
\hline Notation & Definition \\
\hline$A(r \leq R)$ & Total area of all stress perturbations with radius $\leq R$. See equation 47 . \\
\hline$c\left(m_{0}, m_{1}\right)$ & $\begin{array}{l}\text { Filter kernel, used during post-processing to apply a low-pass filter to the } \\
\text { stress field. See equation } 286 \text { in appendix G. }\end{array}$ \\
\hline$D_{c}$ & $\begin{array}{l}\text { Slip-weakening critical distance, that is, the amount of slip over which the } \\
\text { friction drops from its static value to its dynamic value. }\end{array}$ \\
\hline$d\left(m_{0}, m_{1}\right)$ & $\begin{array}{l}\text { Kernel, used during post-processing to select a hypocenter location with } \\
\text { high local stress. See equation } 290 \text { in appendix G. }\end{array}$ \\
\hline$E\left(k_{0}, k_{1}\right)$ & $\begin{array}{l}\text { Elastic energy of the basis displacement field for wave vector }\left(k_{0}, k_{1}\right) \text {. } \\
\text { See equation } 15 \text {. }\end{array}$ \\
\hline$E_{\max }\left(k_{0}, k_{1}\right)$ & $\begin{array}{l}\text { Maximum allowed elastic energy of the basis displacement field for wave } \\
\text { vector }\left(k_{0}, k_{1}\right) \text {; when applying state restriction. See equation } 54 \text {. }\end{array}$ \\
\hline$E_{\text {total }}$ & $\begin{array}{l}\text { Total elastic energy of a linear combination of the basis displacement } \\
\text { fields. See equation } 16 .\end{array}$ \\
\hline $\mathcal{E}\left(k_{0}, k_{1}\right)$ & $\begin{array}{l}\text { Elastic energy density of the basis displacement field for wave vector } \\
\left(k_{0}, k_{1}\right) . \text { Implicitly a function of position in three-dimensional space. See } \\
\text { equation } 130 \text { in appendix B. }\end{array}$ \\
\hline$\varepsilon_{\text {total }}$ & $\begin{array}{l}\text { Total elastic energy density for a linear combination of the basis } \\
\text { displacement fields. Implicitly a function of position in three-dimensional } \\
\text { space. See equation } 147 \text { in appendix B. }\end{array}$ \\
\hline$F\left(k_{0}, k_{1}\right)$ & $\begin{array}{l}\text { Amplitude of basis displacement field for wave vector }\left(k_{0}, k_{1}\right) \text {. See } \\
\text { equations } 8-10 \text {. }\end{array}$ \\
\hline$F_{\max }\left(k_{0}, k_{1}\right)$ & $\begin{array}{l}\text { Maximum allowed amplitude of the basis displacement field for wave } \\
\text { vector }\left(k_{0}, k_{1}\right) ; \text { when applying state restriction. See equation } 53 \text {. }\end{array}$ \\
\hline$F_{\mathrm{NUKE}}$ & $\begin{array}{l}\text { Nucleation factor, which is a multiplier applied to the Day radius to obtain } \\
\text { the radius of the zone of reduced fracture energy. Used in the two-stage } \\
\text { nucleation method. See equation } 293 \text { in appendix G. }\end{array}$ \\
\hline$g(k)$ & $\begin{array}{l}\text { Energy rolloff function. Gives the expected value of the energy } E\left(k_{0}, k_{1}\right) \\
\text { of a basis displacement field, as a function of the scalar wavenumber } k \text {. } \\
\text { See equations } 31,49 \text {, and } 51 \text {. }\end{array}$ \\
\hline$H$ & Variable used in calculating $E_{\max }\left(k_{0}, k_{1}\right)$. See equation 56. \\
\hline$\left(k_{0}, k_{1}\right)$ & Wave vector in the fault plane, associated with the pattern of fault slip. \\
\hline$k_{0}$ & Wave number in the vertical (along-dip) direction along the fault plane. \\
\hline
\end{tabular}




\begin{tabular}{|c|c|}
\hline Notation & Definition \\
\hline$k_{1}$ & $\begin{array}{l}\text { Wave number in the horizontal (along-strike) direction along the fault } \\
\text { plane. }\end{array}$ \\
\hline$k$ & Scalar wave number, $k^{2}=k_{0}^{2}+k_{1}^{2}$. \\
\hline$k_{0}^{*}$ & $\begin{array}{l}\text { Nyquist wave number in the vertical (along-dip) direction. See equation } \\
62 .\end{array}$ \\
\hline$k_{1}^{*}$ & $\begin{array}{l}\text { Nyquist wave number in the horizontal (along-strike) direction. See } \\
\text { equation } 63 .\end{array}$ \\
\hline$L_{0}$ & $\begin{array}{l}\text { Fault size vertically. Also the vertical period of the periodic boundary } \\
\text { conditions. }\end{array}$ \\
\hline$L_{1}$ & $\begin{array}{l}\text { Fault size horizontally. Also the horizontal period of the periodic } \\
\text { boundary conditions. }\end{array}$ \\
\hline$m_{0}$ & Integer identifying a grid point in the vertical direction. See equation 58. \\
\hline$m_{1}$ & $\begin{array}{l}\text { Integer identifying a grid point in the horizontal (along-strike) direction. } \\
\text { See equation } 59 .\end{array}$ \\
\hline$n_{0}$ & $\begin{array}{l}\text { Integer representing a number of periods in the vertical direction. See } \\
\text { equations } 17 \text { and } 60 .\end{array}$ \\
\hline$n_{1}$ & $\begin{array}{l}\text { Integer representing a number of periods in the horizontal (along-strike) } \\
\text { direction. See equations } 17 \text { and } 61 .\end{array}$ \\
\hline$N_{0}$ & Integer representing a number of grid points in the vertical direction. \\
\hline$N_{1}$ & $\begin{array}{l}\text { Integer representing a number of grid points in the horizontal (along- } \\
\text { strike) direction. }\end{array}$ \\
\hline$N_{\mathrm{EQ}}(r)$ & $\begin{array}{l}\text { Rate of occurrence of earthquakes with radius between } r \text { and } r+d r \text {. See } \\
\text { equation } 42 \text {. }\end{array}$ \\
\hline$N_{\mathrm{SP}}(r)$ & $\begin{array}{l}\text { Number of stress perturbations with radius between } r \text { and } r+d r \text { that } \\
\text { exist at an instant of time. See equation } 46 \text {. }\end{array}$ \\
\hline$Q\left(x_{0}, x_{1}\right)$ & $\begin{array}{l}\text { Selection template. Used in the selection algorithm, it identifies the } \\
\text { portions of the fault surface where high or low stress is desired. }\end{array}$ \\
\hline$R_{D}$ & $\begin{array}{l}\text { Day radius, the minimum radius a rupture must attain so that further } \\
\text { expansion of the rupture is energetically favorable. See equation } 76 \text {. Also } \\
\text { see equations } 294 \text { and } 295 \text { in appendix G. }\end{array}$ \\
\hline$R_{\mathrm{ZFR}}$ & $\begin{array}{l}\text { Radius of the zone of forced rupture, used in the two-stage nucleation } \\
\text { method. See equation } 77 .\end{array}$ \\
\hline$R_{\mathrm{ZRFE}}$ & $\begin{array}{l}\text { Radius of the zone of reduced fracture energy, used in the two-stage } \\
\text { nucleation method. See equation } 78 \text {. Also see equation } 293 \text { in appendix G. }\end{array}$ \\
\hline$R\left(k_{0}, k_{1}\right)$ & $\begin{array}{l}\text { Amplitude of generalized basis displacement field for wave vector } \\
\left(k_{0}, k_{1}\right) \text {. See equations } 82-84 \text { in appendix B. }\end{array}$ \\
\hline
\end{tabular}




\begin{tabular}{|c|c|}
\hline Notation & Definition \\
\hline$R\left(x_{0}, x_{1}\right)$ & $\begin{array}{l}\text { Rotation template, used during post-processing to decide whether or not to } \\
\text { rotate the stress field by } 180 \text { degrees. See equation } 285 \text { in appendix G. }\end{array}$ \\
\hline$u_{i}\left(x_{0}, x_{1}, x_{2}\right)$ & $\begin{array}{l}\text { Basis displacement field, which is an approximate solution to the } \\
\text { elastostatic fault slip problem with sinusoidal fault slip. Displacement in } \\
\text { coordinate direction } i=0,1,2 \text { as a function of position in space. There is a } \\
\text { separate basis displacement field for each admissible wave vector, hence } \\
u_{i} \text { is also implicitly a function of wave vector }\left(k_{0}, k_{1}\right) \text {. See equations } 8- \\
10 \text {. }\end{array}$ \\
\hline$u_{i}^{\text {total }}$ & $\begin{array}{l}\text { Total displacement field, obtained by forming a linear combination of the } \\
\text { basis displacement fields. Implicitly a function of position in three- } \\
\text { dimensional space. See equation } 146 \text { in appendix B. }\end{array}$ \\
\hline$U$ & Random variable, uniformly distributed between 0 and 1. \\
\hline$V$ & Random variable, uniformly distributed between 0 and 1. \\
\hline$\left(x_{0}, x_{1}, x_{2}\right)$ & Coordinates in three-dimensional space. \\
\hline$x_{0}$ & Vertical coordinate. \\
\hline$x_{1}$ & Horizontal coordinate along-strike. \\
\hline$x_{2}$ & Horizontal coordinate perpendicular to the fault plane. \\
\hline$X_{K}$ & $\begin{array}{l}\text { Real-valued random variable that has the expected one-point statistics of } \\
\text { the filtered stress field } \tau_{\text {total }}\left(x_{0}, x_{1} ; k \geq K\right) \text {. See equation } 187 \text { in appendix } \\
\text { D. }\end{array}$ \\
\hline$Z\left(k_{0}, k_{1}\right)$ & $\begin{array}{l}\text { Plane wave amplitude for the shear stress generated by the basis } \\
\text { displacement field for wave vector }\left(k_{0}, k_{1}\right) \text {. See equation } 182 \text { in appendix } \\
\text { D. }\end{array}$ \\
\hline$\alpha$ & $\begin{array}{l}\text { Decay exponent of generalized basis displacement field. Implicitly a } \\
\text { function of position } x_{2} \text { perpendicular to the fault plane and scalar } \\
\text { wavenumber } k \text {. See equation } 90 \text { in appendix B. }\end{array}$ \\
\hline$\beta$ & $\begin{array}{l}\text { In thermodynamics, a parameter inversely proportional to temperature. In } \\
\text { fault mechanics, a parameter that determines the correlation length and the } \\
\text { overall level of stress fluctuations. }\end{array}$ \\
\hline$\varepsilon_{i j}$ & Strain tensor. Implicitly a function of position in three-dimensional space. \\
\hline$\zeta$ & $\begin{array}{l}\text { Parameter appearing in the energy rolloff function } g(k) \text {, adjusted to set } \\
\text { the correlation length. See equations } 49 \text { and } 51 \text {. }\end{array}$ \\
\hline$\eta$ & Parameter appearing in the energy rolloff function $g(k)$. See equation 51. \\
\hline$\theta$ & $\begin{array}{l}\text { Complex phase of the convolution }\left(Q \star \tau_{\text {total }}^{\prime}\right)\left(\tilde{x}_{0}, \tilde{x}_{1}\right) \text { at the point where } \\
\text { its absolute value is maximized. See equation } 74 \text {. }\end{array}$ \\
\hline
\end{tabular}




\begin{tabular}{|c|c|}
\hline Notation & Definition \\
\hline$\Lambda$ & $\begin{array}{l}\text { Correlation length. Defined to be } \Lambda=2 \pi / \hat{k} \text {, where } \hat{k} \text { is chosen to } \\
\text { maximize the value of } g(\hat{k}) \hat{k}^{2} \text {. }\end{array}$ \\
\hline$\lambda$ & Volumetric Lame parameter. \\
\hline$\mu$ & Shear modulus. Also the shear Lame parameter. \\
\hline$\Phi\left(k_{0}, k_{1}\right)$ & $\begin{array}{l}\text { Phase of basis displacement field for wave vector }\left(k_{0}, k_{1}\right) \text {. See equations } \\
8-10 \text {. }\end{array}$ \\
\hline$\psi$ & $\begin{array}{l}\text { Total phase of generalized basis displacement field. Implicitly a function } \\
\text { of position }\left(x_{0}, x_{1}\right) \text { on the fault plane and wave vector }\left(k_{0}, k_{1}\right) \text {. See } \\
\text { equation } 89 \text { in appendix B. }\end{array}$ \\
\hline$\sigma_{i j}$ & Stress tensor. Implicitly a function of position in three-dimensional space. \\
\hline$\sigma$ & $\begin{array}{l}\text { Normal stress acting on the fault plane. Implicitly a function of position on } \\
\text { the two-dimensional fault plane. }\end{array}$ \\
\hline$\tau$ & $\begin{array}{l}\text { Shear stress acting on the fault plane. Implicitly a function of position on } \\
\text { the two-dimensional fault plane. We take the shear stress to be oriented } \\
\text { along-strike. }\end{array}$ \\
\hline$\tau\left(k_{0}, k_{1}\right)$ & $\begin{array}{l}\text { Shear stress acting on the fault plane, due to the basis displacement field } \\
\text { for wave vector }\left(k_{0}, k_{1}\right) \text {. It is implicitly a function of position }\left(x_{0}, x_{1}\right) \text { on } \\
\text { the fault plane. See equation } 20 \text {. }\end{array}$ \\
\hline$\tau_{\mathrm{rms}}^{2}\left(k_{0}, k_{1}\right)$ & $\begin{array}{l}\text { Mean-square value of the shear stress acting on the fault plane, due to the } \\
\text { basis displacement field for wave vector }\left(k_{0}, k_{1}\right) \text {. See equation } 21 \text {. }\end{array}$ \\
\hline$\tau_{\text {total }}\left(x_{0}, x_{1}\right)$ & $\begin{array}{l}\text { Total shear stress acting on the fault plane, due to a linear combination of } \\
\text { the basis displacement fields. See equation } 68 \text {. }\end{array}$ \\
\hline$\tau_{\text {total }}^{2}$ & $\begin{array}{l}\text { Mean-square value of the shear stress acting on the fault plane, due to a } \\
\text { linear combination of the basis displacement fields. See equation } 22 \text {. }\end{array}$ \\
\hline$\tau_{\text {total }}^{\prime}\left(x_{0}, x_{1}\right)$ & $\begin{array}{l}\text { Complex-valued total shear stress acting on the fault plane, due to a linear } \\
\text { combination of the basis displacement fields. Used in the selection } \\
\text { algorithm. See equation } 72 \text {. }\end{array}$ \\
\hline$\tau_{\text {total }}\left(x_{0}, x_{1} ; k \geq K\right)$ & $\begin{array}{l}\text { Total shear stress acting on the fault plane, due to a linear combination of } \\
\text { the basis displacement fields, for wavenumbers } \geq K \text {. See equation } 185 \text { in } \\
\text { appendix D. }\end{array}$ \\
\hline$\tau_{\text {total }}^{2}(k \geq K)$ & $\begin{array}{l}\text { Mean-square value of the shear stress acting on the fault plane, due to a } \\
\text { linear combination of the basis displacement fields, for wavenumbers } \geq \\
K \text {. See equation } 186 \text { in appendix D. }\end{array}$ \\
\hline$\Omega\left(x_{0}\right)$ & $\begin{array}{l}\text { Depth conditioning function, which systematically reduces the shear stress } \\
\text { with increasing depth. See equation } 69 \text {. Also see equation } 296 \text { in appendix } \\
\text { G. }\end{array}$ \\
\hline
\end{tabular}


Table 2. Summary of thermodynamic analogies.

The following table summarizes our analogy between thermodynamics and earthquake mechanics. Each row in the table lists a concept from thermodynamics and the analogous concept for earthquake faults.

\begin{tabular}{|c|c|}
\hline Thermodynamic concept & Earthquake fault concept \\
\hline Physical system. & $\begin{array}{l}\text { A planar fault, with periodic boundary } \\
\text { conditions along-strike and along-dip, and the } \\
\text { surrounding elastic medium. }\end{array}$ \\
\hline $\begin{array}{l}\text { Micro-state-A complete specification of the } \\
\text { system's microscopic state. }\end{array}$ & $\begin{array}{l}\text { A pattern of slip on the fault surface and the } \\
\text { resulting distortion of the surrounding elastic } \\
\text { medium. }\end{array}$ \\
\hline Energy of a micro-state. & $\begin{array}{l}\text { Total elastic energy due to the distortion of } \\
\text { the surrounding elastic medium. }\end{array}$ \\
\hline $\begin{array}{l}\text { Degrees of freedom-Partitioning a system } \\
\text { into a set of subsystems, so that the energy of } \\
\text { the complete system equals the sum of the } \\
\text { energies of the subsystems. Each subsystem is } \\
\text { a degree of freedom. }\end{array}$ & $\begin{array}{l}\text { Orthogonal basis displacement fields- } \\
\text { Decomposing an arbitrary pattern of slip on } \\
\text { the fault into a sum of basis displacement } \\
\text { fields, so that the total elastic energy of the } \\
\text { complete slip pattern is equal to the sum of } \\
\text { the elastic energies of the basis displacement } \\
\text { fields. }\end{array}$ \\
\hline $\begin{array}{l}\text { Density of states-Number of micro-states } \\
\text { per unit volume of phase space, or per unit } \\
\text { energy interval. }\end{array}$ & $\begin{array}{l}\text { Density of states-Number of basis } \\
\text { displacement fields with wave vectors that } \\
\text { satisfy periodic boundary conditions, per unit } \\
\text { volume of wave-vector space. }\end{array}$ \\
\hline $\begin{array}{l}\text { Equipartition theorem-Each degree of } \\
\text { freedom has the same expected energy (under } \\
\text { certain conditions). }\end{array}$ & $\begin{array}{l}\text { Equipartition theorem-Each basis } \\
\text { displacement field has the same expected } \\
\text { elastic energy (under certain conditions). }\end{array}$ \\
\hline $\begin{array}{l}\text { Ultraviolet divergence-A mathematical } \\
\text { divergence that arises in systems with } \\
\text { infinitely many short-wavelength degrees of } \\
\text { freedom, because equipartition then predicts } \\
\text { infinite short-wavelength energy. For } \\
\text { example, blackbody radiation is predicted to } \\
\text { contain an infinite amount of ultraviolet } \\
\text { radiation. }\end{array}$ & $\begin{array}{l}\text { Ultraviolet divergence-A mathematical } \\
\text { divergence that arises because there are } \\
\text { infinitely many short-wavelength basis } \\
\text { displacement fields, and equipartition then } \\
\text { predicts an infinite amount of short- } \\
\text { wavelength elastic energy and stress. }\end{array}$ \\
\hline
\end{tabular}




\begin{tabular}{|l|l|}
\hline \multicolumn{1}{|c|}{ Thermodynamic concept } & \multicolumn{1}{c|}{ Earthquake fault concept } \\
\hline $\begin{array}{l}\text { Quantization-Reduces the number of micro- } \\
\text { states by requiring that some physical } \\
\text { quantities (such as energy) can only assume } \\
\text { discrete values instead of being continuously } \\
\text { variable. Can resolve an ultraviolet } \\
\text { divergence by reducing the energy in short- } \\
\text { wavelength degrees of freedom to below the } \\
\text { equipartition value. }\end{array}$ & $\begin{array}{l}\text { Finite fault strength-Reduces the number of } \\
\text { acceptable patterns of slip on the fault surface } \\
\text { by requiring that the shear stress cannot } \\
\text { exceed the yield strength of the fault. (We } \\
\text { enforce this rule in the weak, or mean-square, } \\
\text { sense; rather than enforcing it at each point on } \\
\text { the fault surface.) Resolves the ultraviolet } \\
\text { divergence by reducing the energy in short- } \\
\text { wavelength basis displacement fields. }\end{array}$ \\
\hline $\begin{array}{l}\text { Planck distribution-For blackbody radiation, } \\
\text { a formula that specifies how the expected } \\
\text { energy for each degree of freedom falls off } \\
\text { toward zero as the wavenumber increases. }\end{array}$ & $\begin{array}{l}\text { Energy rolloff function-A formula that } \\
\text { specifies how the expected energy for each } \\
\text { basis displacement field falls off toward zero } \\
\text { as the wavenumber increases. }\end{array}$ \\
\hline
\end{tabular}


Figures.

Figure $1 \mathrm{~A}$.

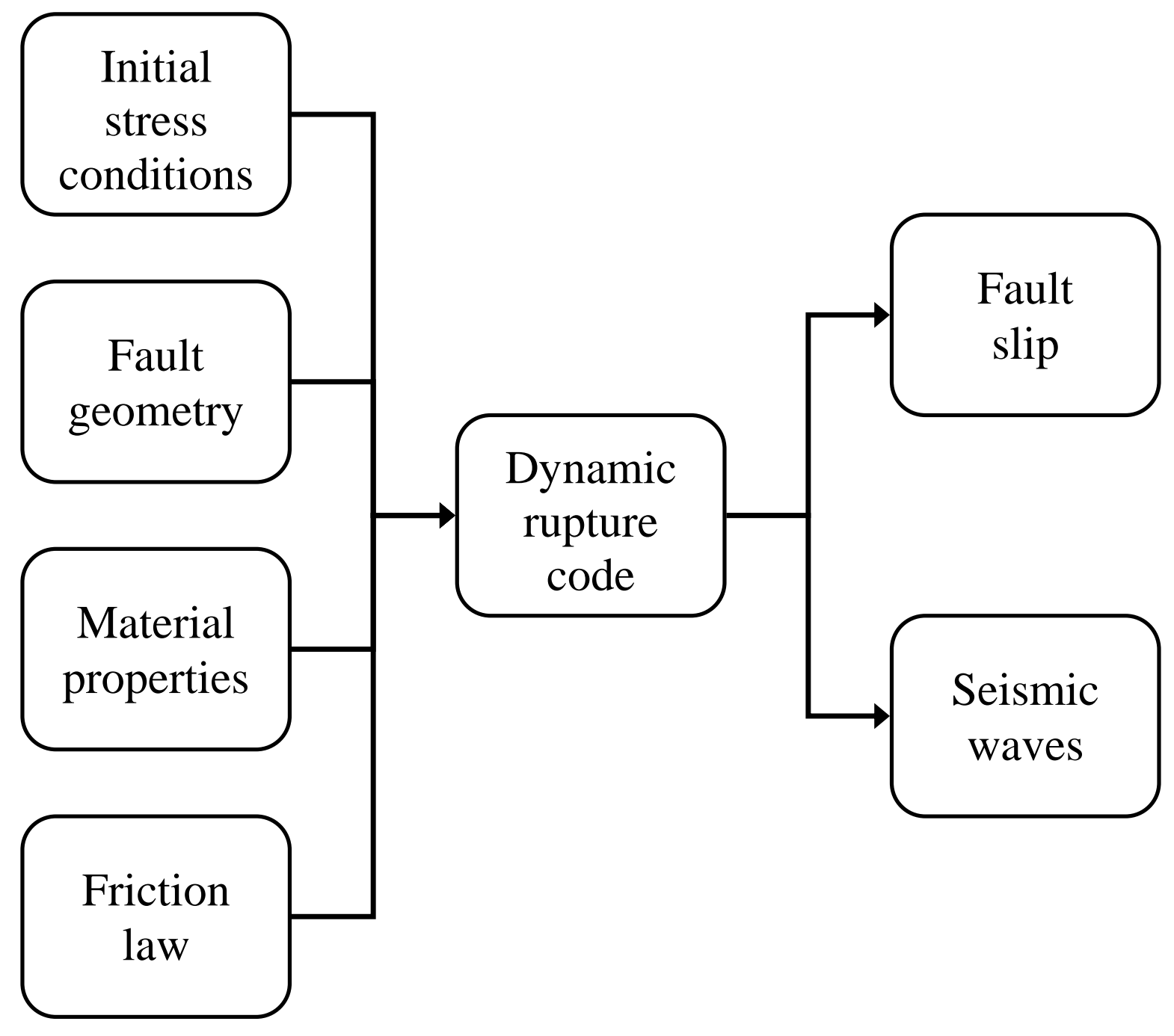


Figure 1B.

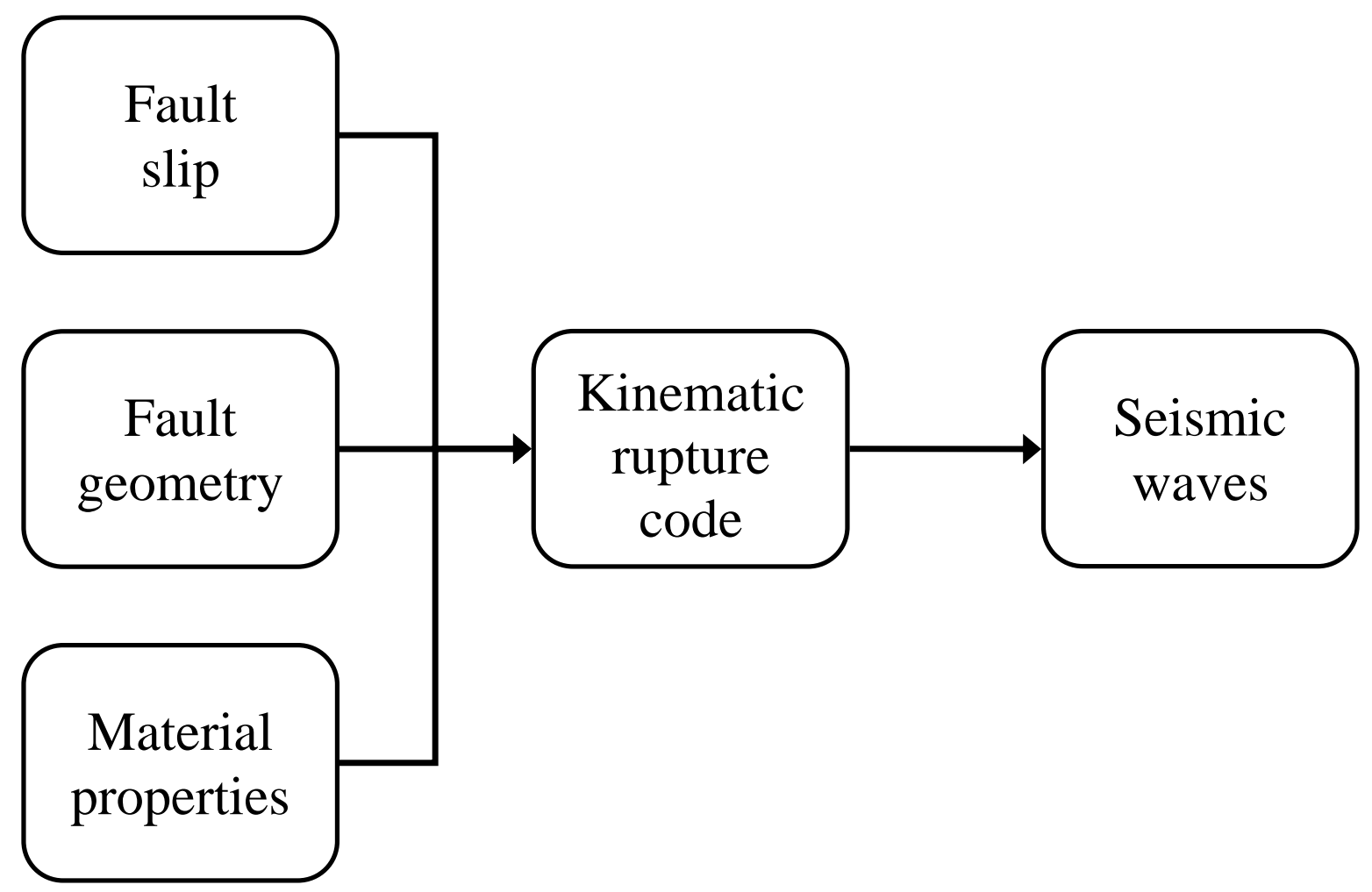

Figure 1. Components of dynamic and kinematic earthquake simulations. (A) A dynamic rupture simulation models the frictional processes that occur on the fault, so the inputs include the friction law and initial stresses, and the simulation code must calculate both fault slip and seismic waves. Our method is a way to inject randomness into the initial stress conditions. (B) A kinematic rupture simulation treats the fault slip as being known a priori, so there is no need to specify a friction law or initial stresses, and the simulation code only needs to calculate the seismic waves. 


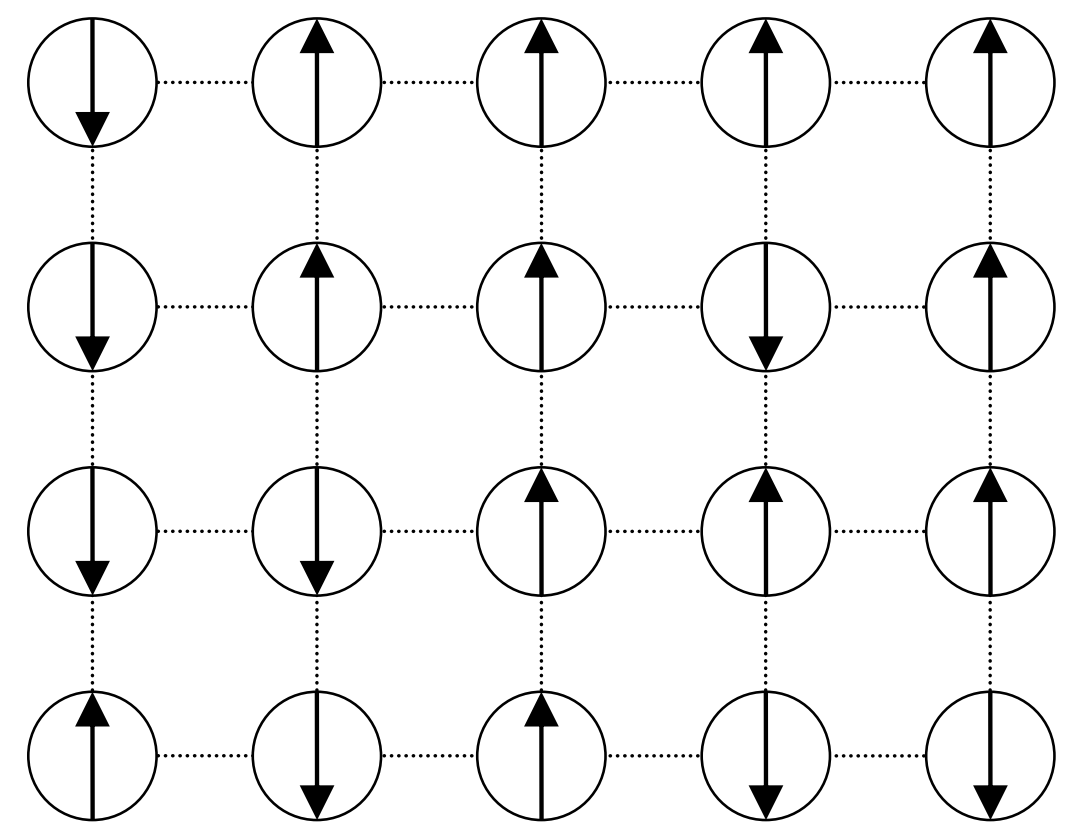

Figure 2. Two-dimensional Ising model. The Ising model is a model of a ferromagnetic material such as iron. The circles represent atoms in a crystal lattice, each of which can interact only with its four nearest neighbors. Each atom has a "spin," shown by an arrow, that can point either "up" or "down." The spin can be thought of as being a small magnet. The interaction energy between two nearest-neighbor atoms depends on whether their spins are parallel or antiparallel. The Ising model illustrates how a system can self-organize into large structures such as magnetic domains. 


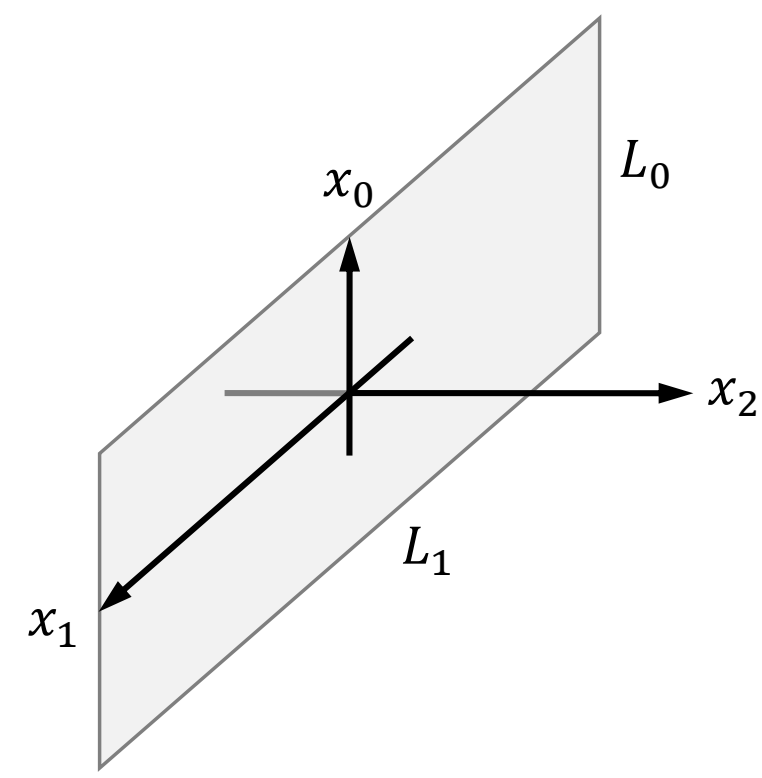

Figure 3. Three-dimensional coordinate system. We assume a vertical strike-slip fault. The fault lies in the $x_{0} x_{1}$-plane, with the $x_{0}$ axis pointing up and the $x_{1}$ axis pointing along-strike. The $x_{2}$ axis is perpendicular to the fault. We impose periodic boundary conditions in the $x_{0}$ and $x_{1}$ directions (but not the $x_{2}$ direction), with periods $L_{0}$ and $L_{1}$, respectively. So the area of the fault is $L_{0} L_{1}$. 


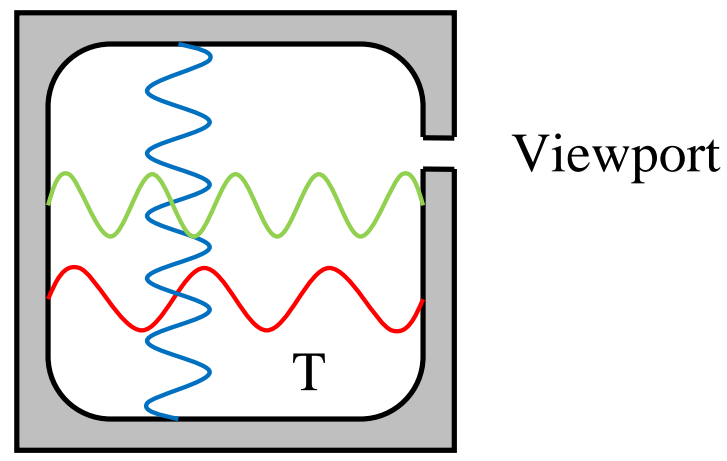

Figure 4. Blackbody radiation. A container is maintained at a temperature $T$, and a small viewport allows the experimenter to observe the electromagnetic radiation inside the container. The problem is to compute the spectrum and energy density as a function of temperature. The equipartition theorem predicts infinite energy density, a difficulty known as an ultraviolet divergence. However, quantum mechanics greatly reduces the number of possible states of the system, with the result that the energy density is rendered finite. 
Figure 5A.

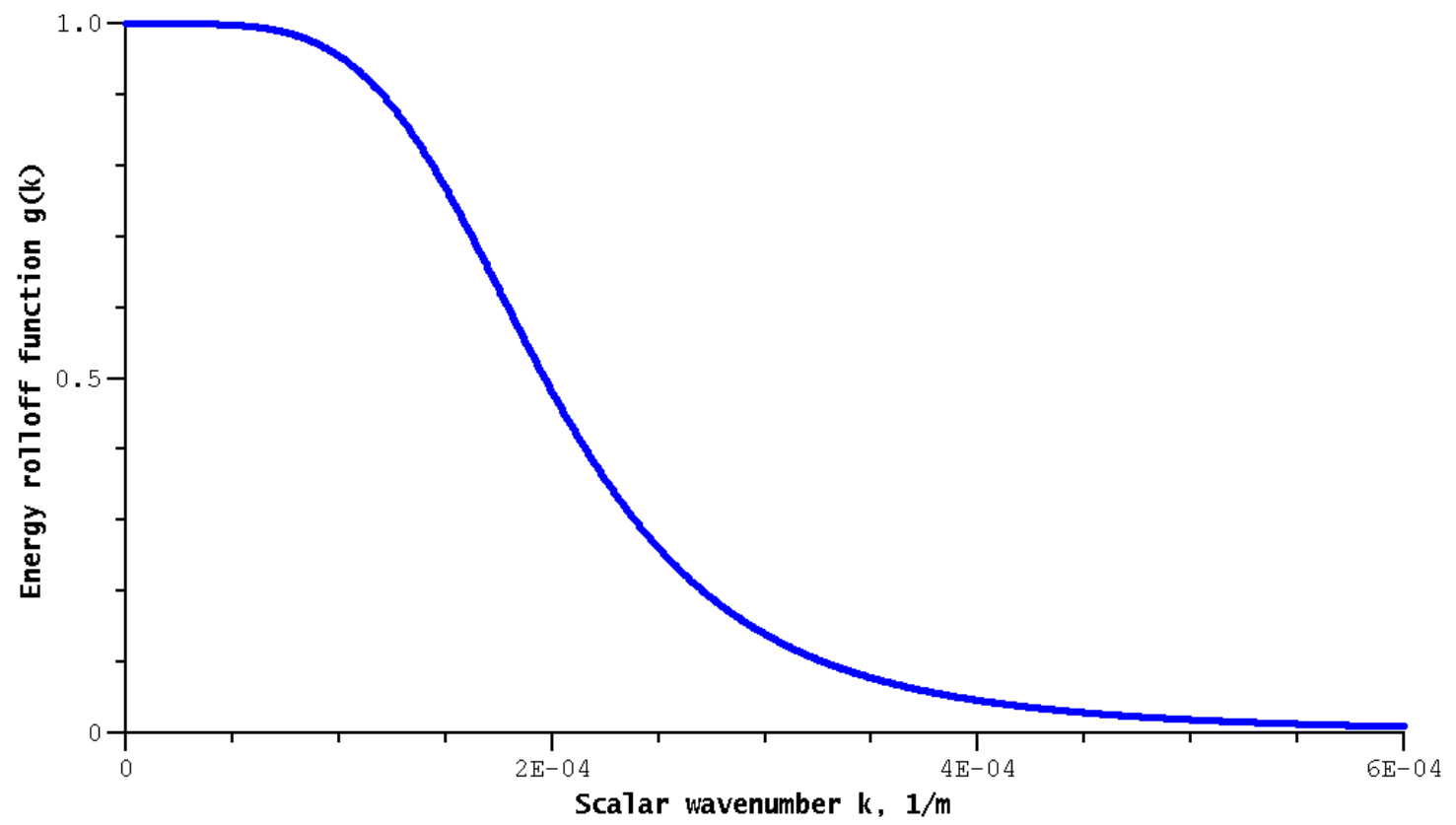


Figure 5B

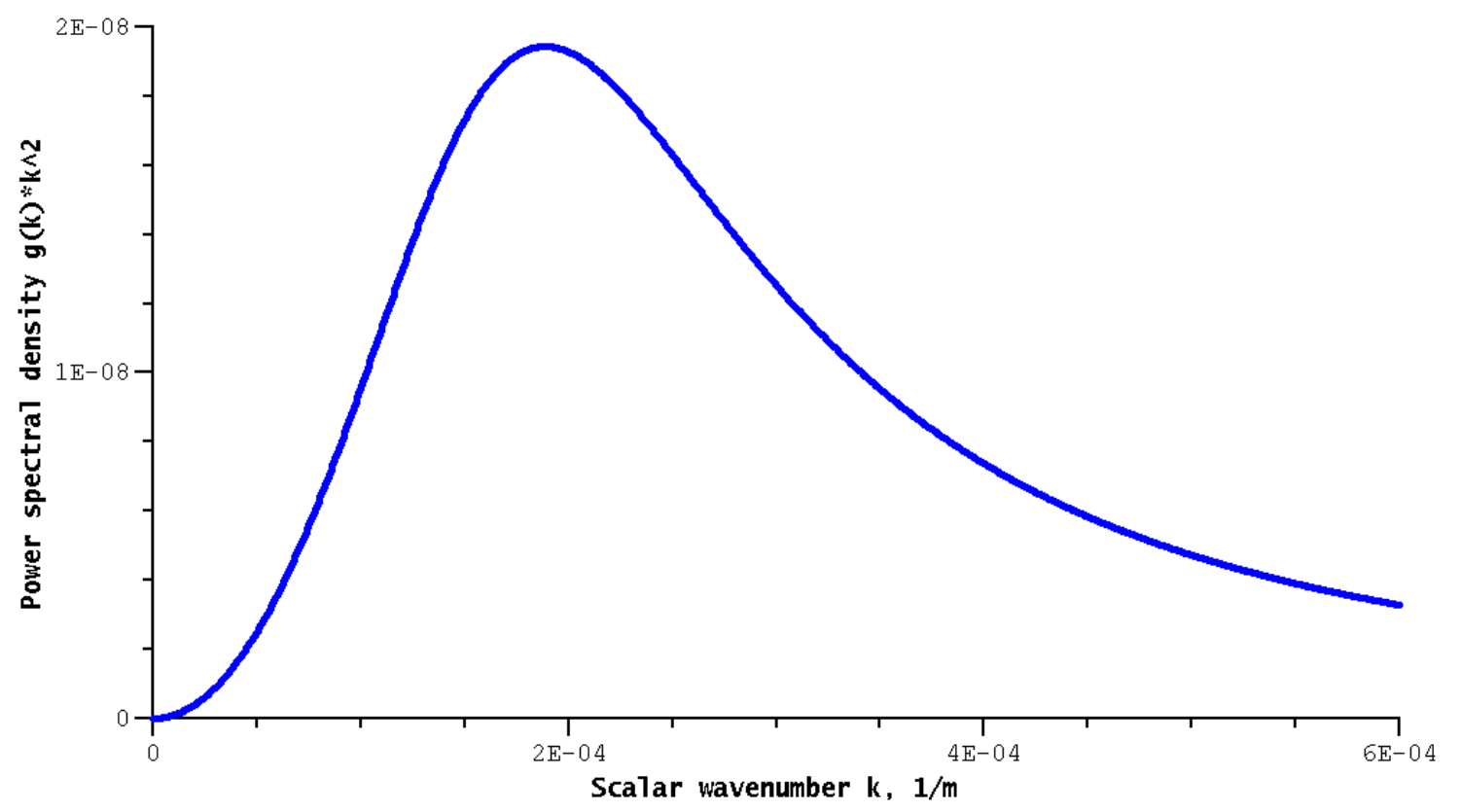

Figure 5. Energy rolloff function. (A) Energy rolloff function $g(k)$ plotted as a function of the scalar wavenumber $k$. The plot begins with $g(0)=1$ and decreases monotonically, approaching 0 in the limit $k \rightarrow \infty$. As explained in the main text, the finite strength of the fault restricts the possible states of the system, and, as a result, the expected energy of a displacement field with wavenumber $k$ is given by $g(k) / \beta$ where $\beta$ is the thermodynamic parameter analogous to reciprocal temperature. By comparison, equipartition predicts that the expected energy is $1 / \beta$ independent of $k$, which leads to an ultraviolet divergence. (B) One-dimensional power spectral density function $g(k) k^{2}$ plotted as a function of $k$. The area under this curve must be finite, for our method to yield a finite level of stress. The maximum value occurs at $k=2 \pi / \Lambda$ where $\Lambda$ is defined to be the correlation length. The plots in this figure use $g(k)=1 /\left(1+(\eta k)^{3}(\log (1+\zeta k))^{2}\right)$ where $\eta=\zeta=6,000$ meters. 
Figure 6A.

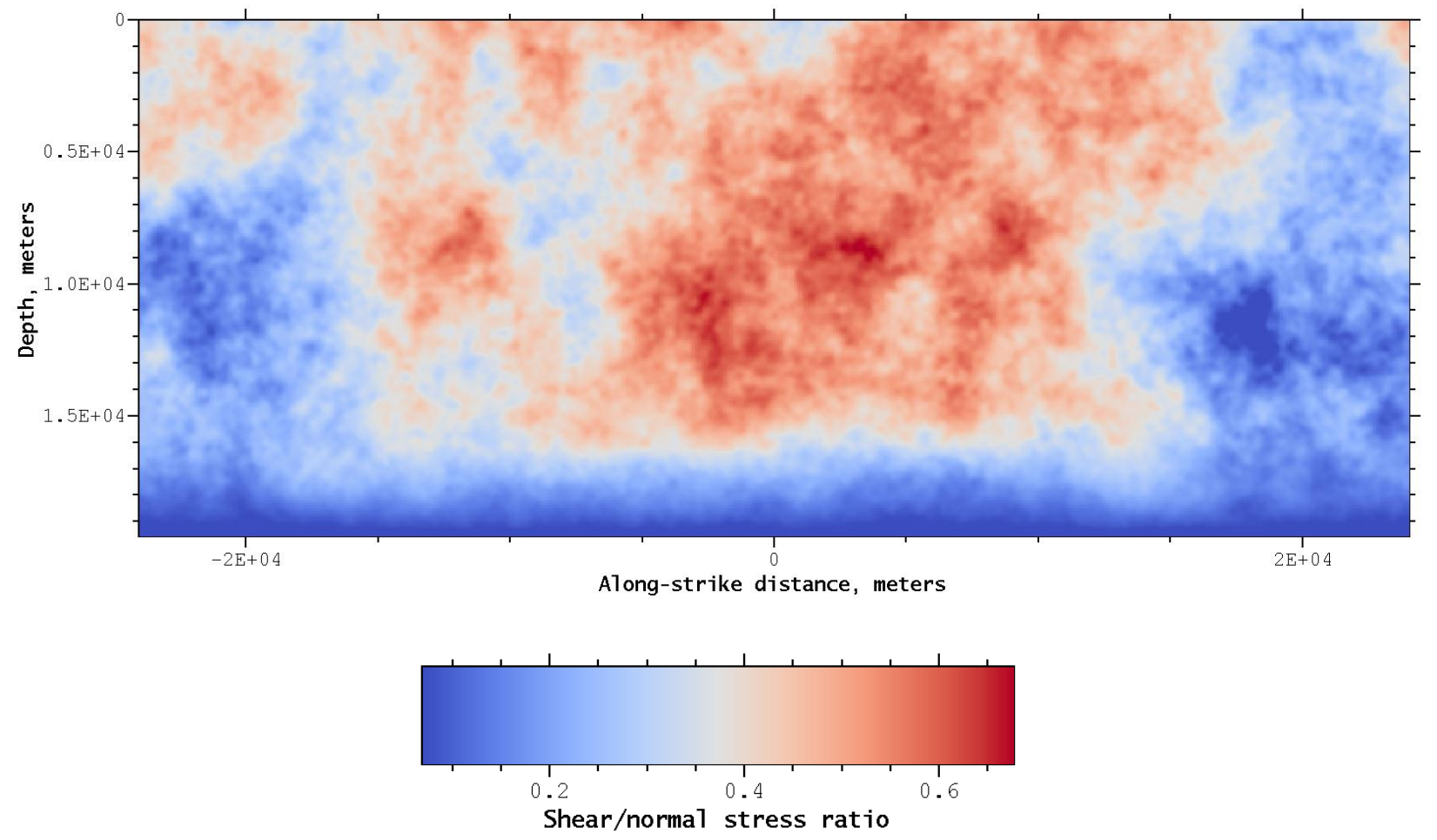


Figure 6B.

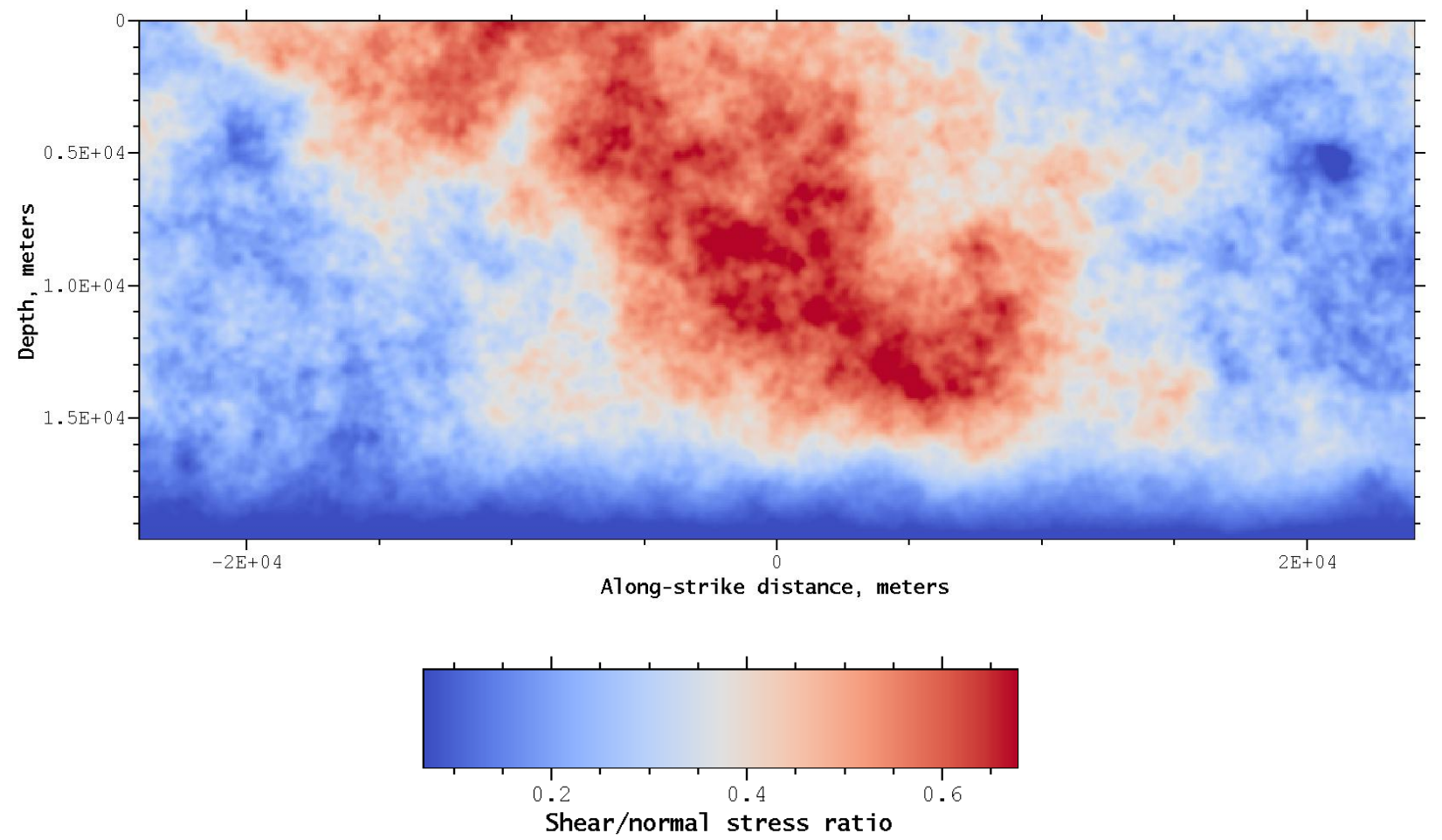

Figure 6. Examples of random stress distributions generated by our thermodynamic method. Levels of stress on the fault surface are shown by varying colors, which indicate the ratio of shear stress divided by normal stress. Rupture extent is limited by the low-stress areas along the sides and bottom of the fault. (A) Stress pattern used in Southern California Earthquake Center Dynamic Rupture Code Validation benchmark TPV16. (B) Stress pattern used in benchmark TPV17. 


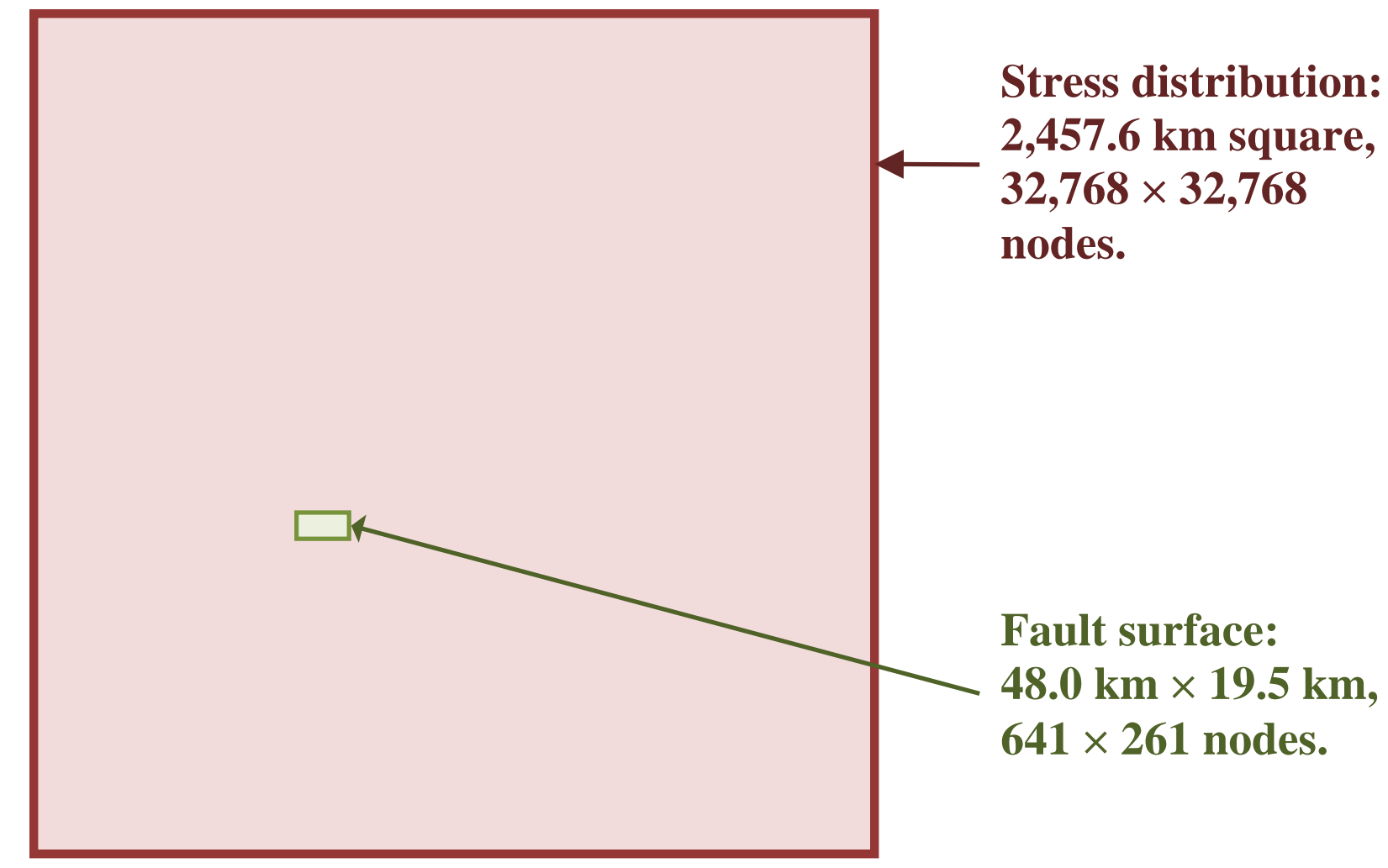

Figure 7. Selection process for controlling rupture extent along-strike. A random stress distribution is generated over an area thousands of times larger than the fault surface. Then, the entire large area is searched to find a portion, the size of the fault surface, which by chance has high stress in the center and low stress at the sides. In this example, the large area is 32,768 grid points square, which is $2,457.6$ kilometers $(\mathrm{km})$ square assuming a grid point spacing of 75 meters $(\mathrm{m})$. The fault area is 641 by 261 grid points, or $48.0 \mathrm{~km}$ by $19.5 \mathrm{~km}$. The figure is not to scale. If drawn to scale, the fault surface would have about one-tenth the area shown. 


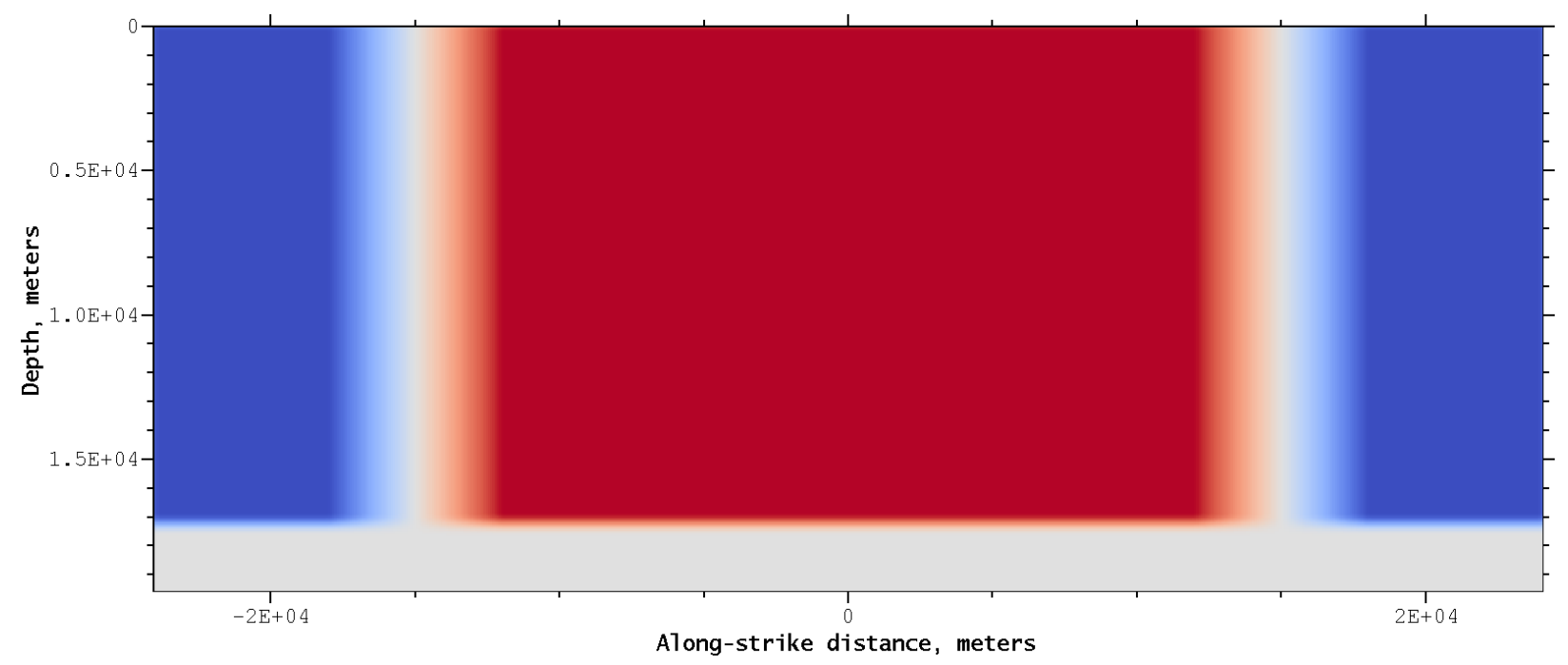

Figure 8. Selection template. Colors show the value of the selection template $Q\left(x_{0}, x_{1}\right)$, which is used in the selection process. It is positive in the area where high stress is desired, in this example the central 30 kilometers $(\mathrm{km})$ of the fault surface. It is negative where low stress is desired, which is to the left and right sides. It is zero where the stress level is unimportant, in this example the bottom $2.5 \mathrm{~km}$ of the fault surface where rupture extent is controlled by depth conditioning. There is a gentle taper between the positive and negative areas, spanning $6 \mathrm{~km}$, to allow for an irregular border between high and low stress areas. The selection template tapers to zero over a span of $0.5 \mathrm{~km}$, part of which lies outside the fault surface (not shown). 
Figure 9A.

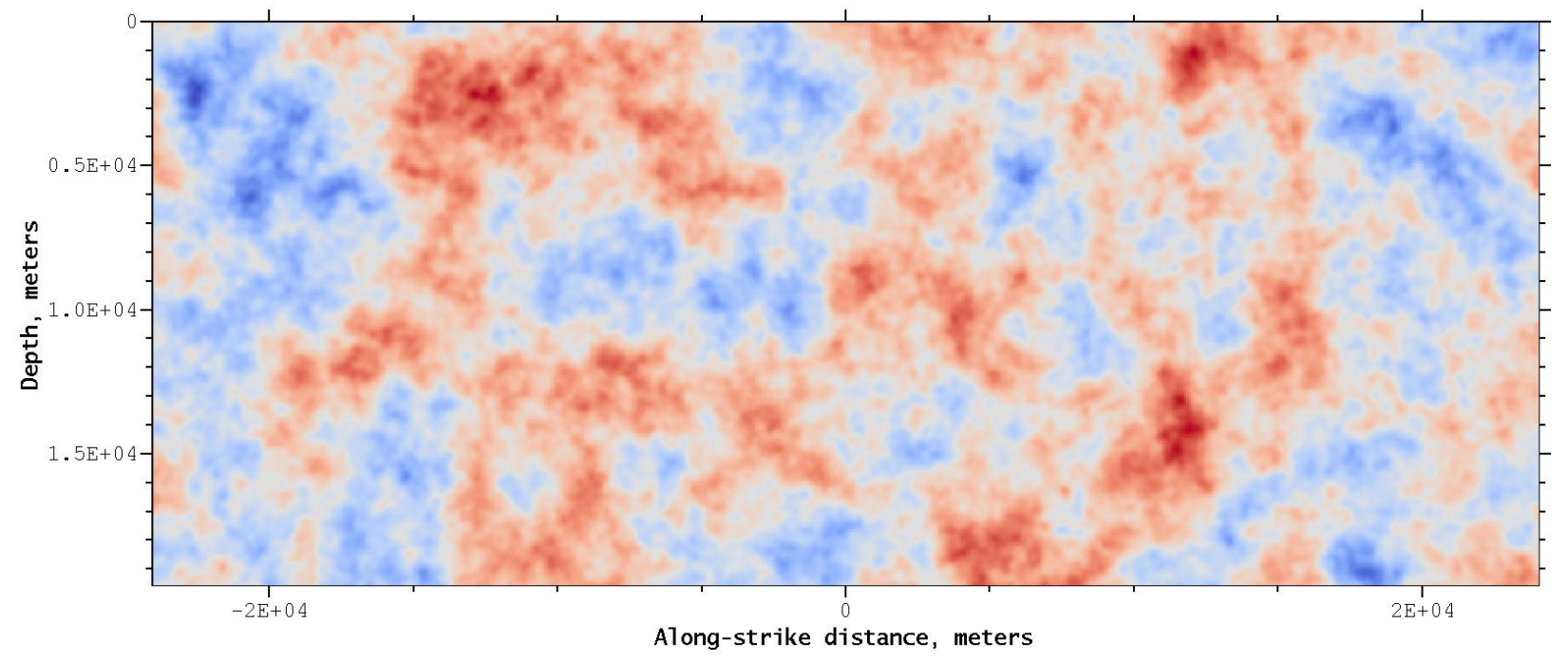

Figure 9B.

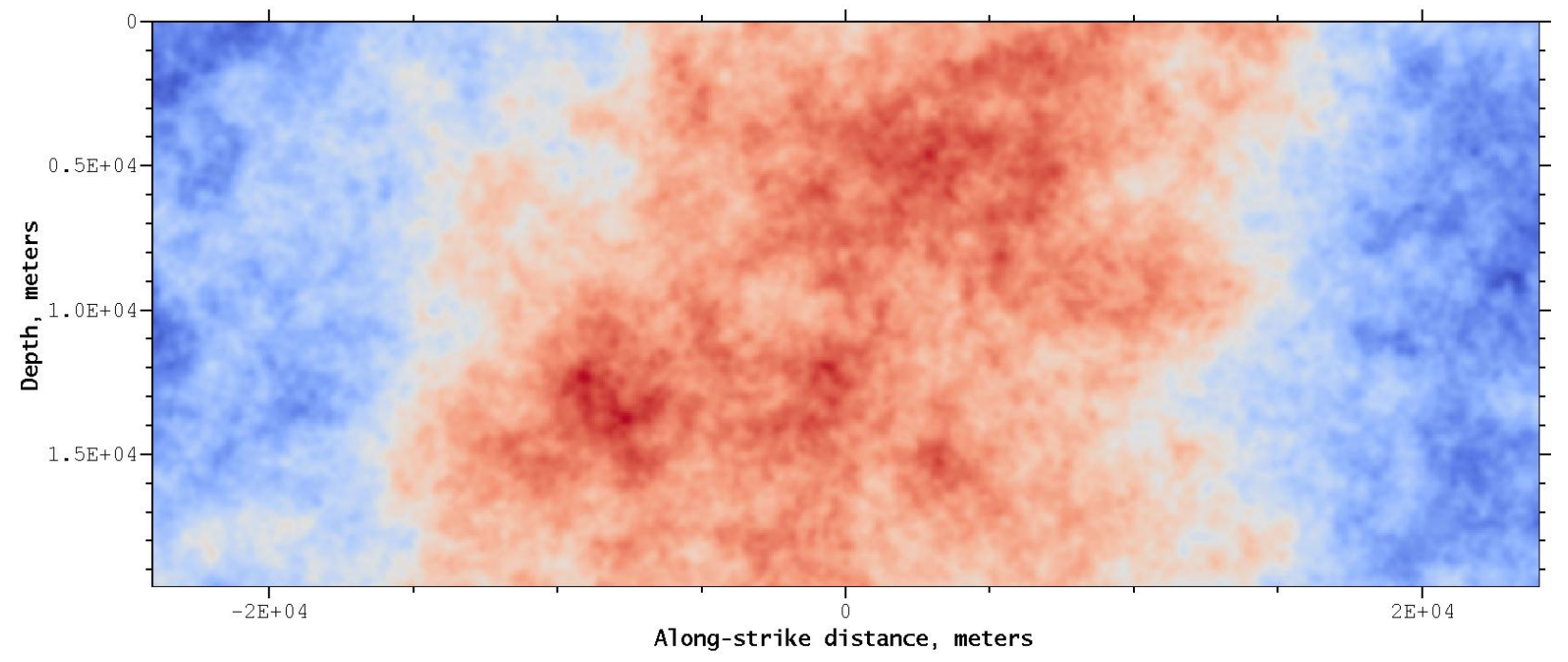


Figure 9C.

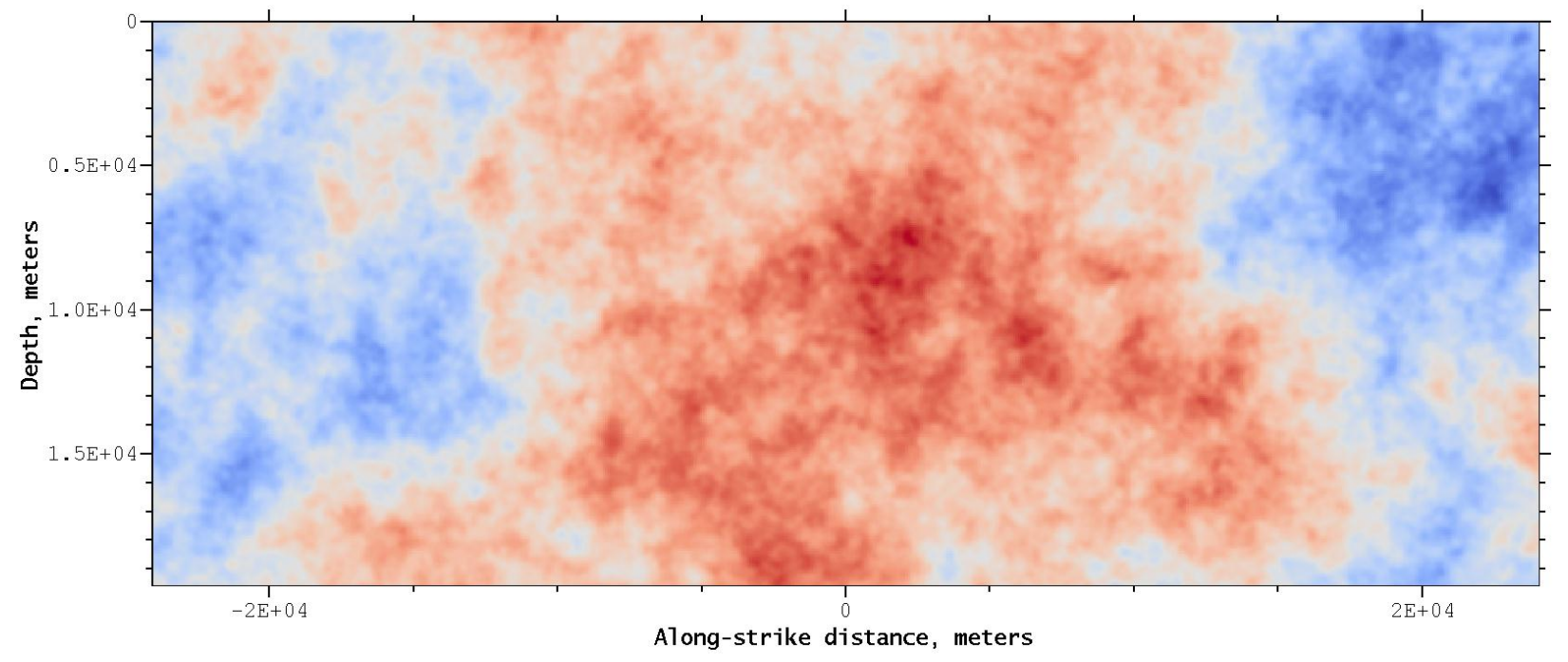

Figure 9. Effect of varying the correlation length. Colors show the level of stress on the fault surface, computed for three different correlation lengths. Images are shown without the use of depth conditioning. (A) Correlation length $=10$ kilometers $(\mathrm{km})$. This is too small, as shown by the fact that it is impossible to find a high-stress area $30 \mathrm{~km}$ across. (B) Correlation length $=100 \mathrm{~km}$. This is too big. Notice that the borders between the high- and low-stress areas are fairly well-defined. (C) Correlation length $=30 \mathrm{~km}$. This is the right size, in this example where we seek a high-stress area $30 \mathrm{~km}$ across. Note the ragged borders between the high- and low-stress areas. Our solution is to choose the smallest correlation length that gives a sufficiently large area of high stress to sustain the simulated earthquake rupture. 


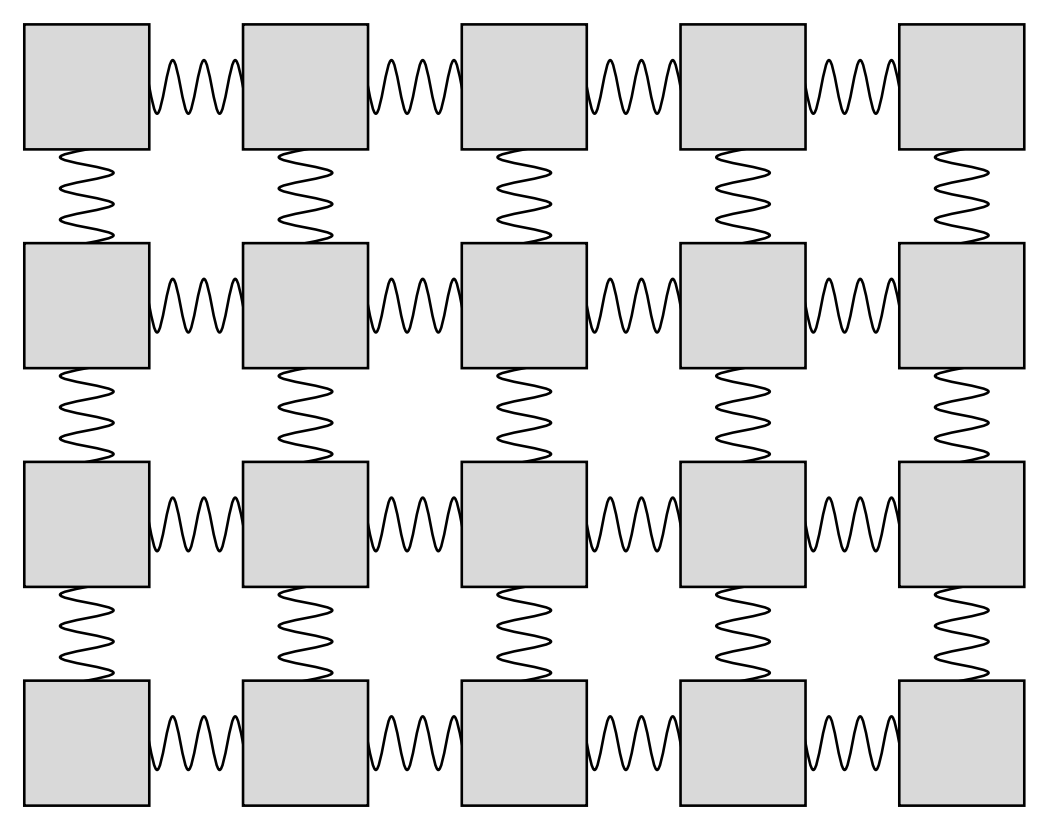

Figure A-1. Slider-block model of an earthquake fault. The fault is modeled as a rectangular array of blocks, each connected by springs to its four nearest neighbors. The blocks can slide within the plane, subject to the forces produced by the springs, and the displacement of a block from its initial position represents fault slip. Each block is also subject to a tectonic driving force, which could be supplied by a fifth spring, which is connected to a rigid plate (not shown in the figure). Although the slider-block model has an obvious similarity to the Ising model, it is not suitable for our purposes because it has incorrect energy. If a sinusoidal displacement pattern is applied to the blocks, the energy stored in the springs is proportional to $k^{2}$, where $k$ is the wavenumber, whereas sinusoidal slip on a fault produces elastic energy proportional to $k$. 